\title{
Calculations of Maximum Allowable Heat Losses for Various Shallow Trench Heat Distribution Systems
}

Jin B. Fang

U.S. DEPARTMENT OF COMMERCE

National Bureau of Standards

National Engineering Laboratory

Center for Building Technology

Building Environment Division

Gaithersburg, MD 20899

November 1987

Prepared for:

Tri-Service Building Materials Committee Headquarters, U.S. Army Corps of Engineers

Washington, DC 20314-1000

U.S. Navy, Naval Facilities Engineering Command

Alexandria, VA 22332-2300

\section{U.S. Air Force, Air Force Engineering and}

Sanices Center

100

.456

$87-3617$

1987

C. 2

$$
\text { Air Force Base, FL 32403-6001 }
$$



Jin B. Fang

U.S. DEPARTMENT OF COMMERCE

National Bureau of Standards

National Engineering Laboratory

Center for Building Technology

Building Environment Division

Gaithersburg, MD 20899

November 1987

Prepared for:

Tri-Service Building Materials Committee Headquarters, U.S. Army Corps of Engineers

Washington, DC 20314-1000

U.S. Navy, Naval Facilities Engineering Command

Alexandria, VA 22332-2300

U.S. Air Force, Air Force Engineering and Services Center

Tyndall Air Force Base, FL 32403-6001

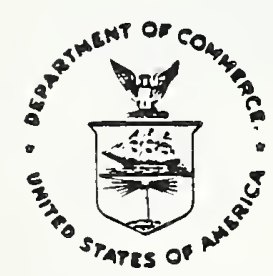

U.S. DEPARTMENT OF COMMERCE, C. William Verity, Secretary NATIONAL BUREAU OF STANDARDS, Ernest Ambler, Director 
$-$ 


\section{ABSTRACT}

The calculation of heat losses for shallow trench underground heat distribution systems was performed using a finite element computer program. The finite element analysis solved a two-dimensional steady-state heat transfer problem for two insulated pipes in a rectangular trench with surrounding soil. A life-cycle cost analysis was performed to determine the cost of construction and annual energy cost associated with pipe heat loss for underground concrete trench systems of different trench dimensions and insulated pipe sizes. Procedures for determining the pipe heat losses associated with the minimum life-cycle cost and the corresponding optimum insulation thickness for shallow trench distribution systems are presented. Based on the results of the economic analysis, the maximum allowable heat losses and the insulation thickness for underground pipes were determined and tabulated for a range of pipe sizes and fluid temperatures, various levels of fuel costs, and for a known undisturbed earth temperature and soil thermal conductivity.

Keywords: district heating and cooling; finite element method; fuel energy cost; heat loss; life-cycle cost analysis; pipe insulation thickness; shallow trench; underground heat distribution system. 
1. Introduction $\ldots \ldots \ldots \ldots \ldots \ldots \ldots \ldots \ldots \ldots \ldots \ldots \ldots \ldots$

2. Pipe Heat Loss Calculations $\ldots \ldots \ldots \ldots \ldots \ldots \ldots \ldots \ldots \ldots \ldots$

3. Comparison Between Finite Element and Thermal Analyzer Programs 6

4. Life-Cycle $\operatorname{Cost} \quad \ldots \ldots \ldots \ldots \ldots \ldots \ldots \ldots \ldots \ldots \ldots \ldots \ldots \ldots \ldots$

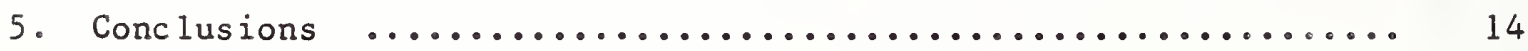

6. Acknowledgment $\ldots \ldots \ldots \ldots \ldots \ldots \ldots \ldots \ldots \ldots \ldots \ldots \ldots \ldots \ldots \ldots \ldots \ldots \ldots$



\section{LIST OF TABLES}

Table I A Comparison of the Pipe Heat Losses Calculated from Temperature Dependent Thermal Conductivity with those Obtained from Constant Thermal Conductivity of Calcium

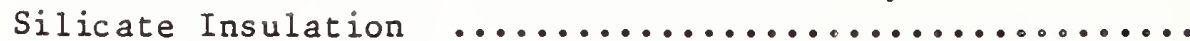

Table 2 Comparisons of Calculated Results from Finite Element Computer Program with Those by the Thermal Analyzer

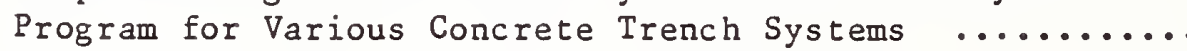

Table 3 Effect of Trench Dimension on Pipe Heat Losses .......

Tables A-1 Maximum Allowable Heat Losses and Economic Insulation through A-13 Thickness for Hot Water Supply and $150 \mathrm{~F}$ Return Pipes and for Energy Cost of $\$ 3$ through $\$ 15 / M B t u$.........

Tables B-1 Maximum Allowable Heat Losses and Economic Insulation through $B-13$ Thickness for Steam Supply and $150 \mathrm{~F}$ Condensate Pipes and for Energy Cost of $\$ 3$ through $\$ 15 / M B t u \quad \ldots \ldots \ldots$.

Tables C-1 Maximum Allowable Heat Losses and Economic Insulation through C-13 Thickness for Hot Water Supply and $250 \mathrm{~F}$ Return Pipes and for Energy Cost of $\$ 3$ through $\$ 15 /$ MBtu 
Figure 1. Concrete Shallow Trench Underground Heat Distribution

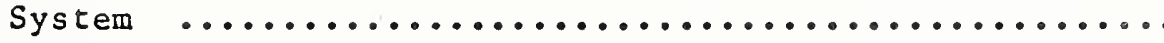

Figure 2. Thermal Conductivity Variation with Temperature for

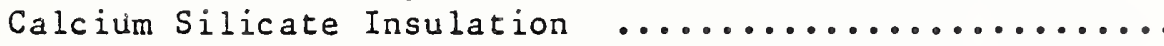

Figures 3 Construction Cost for a Shallow Trench System

through 14. Containing Two 1-inch through 18-inch Insulated Pipes

Figures 15 Construction Cost for a Shallow Trench Systen

through 25. Containing a 2-inch through 18-inch supply and a 1 -inch through 10-inch Return Insulated Pipes ...... 29 

1. Introduction

The production and supply of hot water, chilled water, or steam by a centralized plant is more efficient and economical than production and supply using a number of smaller units. However, this advantage is not possible unless the cost of delivering the working fluid through pipe-lines to the point of use is low. The cost of constructing a distribution pipe-line network generally accounts for the major portion of the total cost of acquiring a district heating and cooling system. In addition, the cperating characteristics of the piping system, such as pipe heat loss, operation reliability and maintenance expense, are of major consideration. An extensive financial loss and intensive labor cost will be involved if a system outage occurs. The heat loss through the underground distribution system consumes a large portion of the system fuel energy cost, and the unavoidable escalation in future fuel prices will make such losses more important. Knowledge of pipe heat loss conditions is essential for initial planning and detailed design, choice of materials, and estimated cost of an underground heat distribution system.

The military installations currently maintain approximately 6,000 miles $(11,110 \mathrm{~km})$ of heat distribution systems [1]. Some of these systems have required repair or replacement due to excessive heat loss resulting from deteriorating thermal pipe-insulation and/or corroding pipes and concuits. Recently, Pan Am [1] and Parsons [2] conducted comparisons of directly buried conduit versus shallow concrete trench heat distribution systems for delivering steam and hot water using life-cycle cost analysis to assess 
their relative merits. The findings of both studies did not agree with each other, especially for the distribution pipe-lines involving large diameter pipes. The discrepancy was probably due to the estinated cost data. However, continued construction of shallow concrete trench systems is anticipated because of their reduced maintenance and repair costs, easy access for inspection and testing, and relatively dry conditions for minimizing possible based cr life-cycle cost analysis was developed at NBS for calculatinc the maximum allowable heat loss for directly buried conduit underground systems [3], and the results were included in the guide specification for nilitary construction, CEGS-15705 [4]. The methods of determining the minimum lifecycle cost heat loss and the optimum insulation thickness for shallow trench systems will-complement the procedure already developed for directly buried conduit systems.

This report presents the procedures to calculate the fuel energy cost associated with pipe heat loss, a set of tables listing the maximum allowable heat loss per unit length of piping section, and the corresponding econoric insulation thickness for heat supply and return pipes installed in shallow trench distribution systems. The minimur life-cycle cost heat loss data are prepared based on a range of pipe sizes, insulation thicknesses, energy prices, soil and insulation thermal condictivities and a known und isturbed earth temperature. This report also presents the estimated inaterial and installation costs for constructing various distribution systems of different trench dimensions and insulated pipe sizes. 
2. Pipe Heat Loss Calculations

The heat transfer process in a rectangular concrete trench containing a pair of insulated circular pipes and the surrounding soil is quite complex due to the involvements of complicated geometries, composite materials, and at least two modes of heat transfer - conduction and convection. Therefore, the solution of this non-linear type of heat transfer problem by exact methods is not possible.

The heat loss per unit length in a concrete shallow trench underground heat distribution system was calculated using a recently developed computer simulation program $[5,6]$. This program has applied the finite element method to a steady-state, two-dimensional, rectangular concrete trench containing two insulated pipes as shown in Figure 1 . A twin pipe system comprised of a heat supply and a return line is modelled in the computer program since it is the most commonly used shallow trench heat distribution system. The heat loss calculations are based on a two-dimensional heat flow and account for heat transfer from the pipe fluid through the pipe insulation, the air space, and the concrete trench walls to the surrounding soil and ambient air. Convective heat transfer in the air space between the pipe and trench wall surfaces is treated by assuming an effective conductance for natural convection in the trench. The surface resistance to convective heat transfer between the ambient air and the ground surface in the vicinity of the shallow trench is treated as being contained in an equivalent soil layer [6]. The effect of radiant exchange between the pipes and trench walls is assumed to be negligible due to low emissivity of the aluminum jacket surface. The boundary conditions are the average temperatures of working fluids 
flowing inside the circuiar pipes, and the undisturbed earth temperatures along the perimeter of the earth region.

The computer program contains a predesigned finite element mesh with 80 nodal points and 130 triangular elements representing the outer boundary earth region, concrete trench walls, top concrete cover, pipe insulation, and the air space between the insulated pipes and the trench walls. The external boundary temperatures including the nodal points, around the outer surfaces of the circular pipes and along the outermost perineter of the outer earth region surrounding the shallow trench system are prescribed from the data input for computer runs. The system of simultaneous equations solves for the remaining nodal point temperatures using the Gausian elimination method. The computer code is written in FORTRAN 77 progranming language and implemented on an IBM personal computer.

The rate of heat loss from an insulated pipe can be obtained using the following equation derived from one-dimensional, steady-state, radial heat conduction in a composite pipe, based on the average temperature difference across a circular cylindrical shell of pipe insulation layer:

$$
q=\frac{(2 \pi) k_{I}\left(T_{i}-T_{0}\right)}{(12) \ln \left(\gamma_{0} / \gamma_{i}\right)}
$$

where $q=$ the heat loss rate per unit length of the insulated pipe, Btu/h f ft $(w / m)$

$$
\begin{aligned}
k_{I}= & \text { the thermal conductivity of insulation material, } \mathrm{Btu} \cdot \mathrm{in} / \mathrm{h} \cdot \mathrm{ft}^{2} \cdot \mathrm{F} \\
& \left(\mathrm{W} / \mathrm{m}^{\circ} \mathrm{K}\right)
\end{aligned}
$$


$\gamma_{0}=$ outside radius of the insulation layer, ft ( $\mathrm{m}$ )

$\gamma_{i}=$ inside radius of the insulation layer, ft ( $\mathrm{m}$ )

$T_{i}$ and $T_{0}=$ the surface temperature of the insulation layer at inner and outer radii, respectively, F (C)

The pipe insulation is the major resistance to heat transfer for an insulated the pipe, the thermal resistance of the pipe wall, and that of the netal jacket are small and can be assumed negligible. Adetailed description and the input data required for executing the computer program, along with the results of some samplecalculations, were sumarized in previous studies [6].

In order to obtain a more realistic prediction of the pipe heat losses, especially ät higher pipe fluid temperatures, a subprogram was added to this finite element computer program to account for the temperature dependency of the pipe-insulation thermal conductivity. The thermal conductivity of calcium silicate, which is resistant to aging during periodical wetting and drying and commoniy used for pipe insulation, as a function of the specinent mean temperature [7] is shown in Figure 2. In this figure, the thermal conductivity increases with increasing temperature. The data used to generate the curve in Figure 2 are stored in a computer subprogram, which is formulated based on the table look-up procedure to provide the remperature dependent thermal conductivity value to be used for each triangular element of pipe-insulation. Since the mean temperatures of the earth surrounding the trench and the pipe insulation are dependent upon the rates of heot transfer through them, an iterative procedure is used along with adjustment of soil and insulation thermal conductivities until the heat loss from the underground pipes achieves a steady-state condition. 
Computer calculations were performed to detemine the effect of thermal conductivity variations with temperature on pipe heat loss for two 6-inch $(150 \mathrm{~mm})$ steel pipes installed side by side in a $4.08 \mathrm{ft}(1.24 \mathrm{~m})$ wide by $2.92 \mathrm{ft}(0.89 \mathrm{~m})$ high concrete trench having $6 \mathrm{in.}(152 \mathrm{~mm})$ thick walls and cover. The top cover was laid flush with ground level. The temperature of hot water flowing in the heat supply pipe was $385 \mathrm{~F}(196 \mathrm{C})$ and the return pipe temperature was $210 \mathrm{~F}(99 \mathrm{C})$. The pipes were insulated with 3.5 in. (89 thick calcium silicate encased in an aluminum jacket, allowing 4 in. (102 mm) of separation between outer surfaces of the carrier pipes. The earth in the vicinity of the shallow trench had annual average temperature of $56 \mathrm{~F}(13 \mathrm{C})$ and themal conductivity of $15 \mathrm{Btu} \cdot \mathrm{in} / \mathrm{h} \cdot \mathrm{ft}^{2} \cdot \mathrm{F}\left(2.16 \mathrm{~W} / \mathrm{m}^{\circ} \mathrm{K}\right)$. A comparison of the heat loss values calculated from temperature dependent insulation thermal conductivity with those obtained from the constant thermal conductivity of $0.44 \mathrm{Btu} \cdot \mathrm{in} / \mathrm{h} \cdot \mathrm{ft}^{2} \cdot \mathrm{F}(0.063 \mathrm{~V} / \mathrm{m} \cdot \mathrm{K})$ for various insulation thicknesses is given in Table 1. For this sample case, the temperature dependent thermal conductivity gave approximately 3 to $4 \%$ greater total heat loss values compared to the constant thermal conductivity.

3. Comparison Between Finite Element and Thermal Analyzer Programs

The thermal analyzer computer program, TASTAP, developed based on the application of finite difference technique was recently employed for calculating the pipe heat losses of shallow trench and directiy buried conduit heat distribution syster.s [2]. The capabilities of this finite 
difference conputer simulation program were described briefly in Parsons' report [2]. There is a lack of field data on pipe heat loss, temperature distributions of trench air, concrete walls, and soil in the vicinity of underground systems, which are available to be utilized for validation of the predictive methods. Some calculations using the finite element computer program were carried out on typical shallow trench systems with different pipe sizes and fluid temperatures, and the results were compared with the predictions made from the thermal analyzer program TASTAP. An average ground temperature of $55 \mathrm{~F}(12.8 \mathrm{C}$ ), a $6 \mathrm{in.}(152 \mathrm{~mm}$ ) thick trench walls and cover, and the thermal conductivities of soil, concrete and pipe insulation equal to 15,8 , and $0.444 \mathrm{Btu} \cdot \mathrm{in} / \mathrm{h} \cdot \mathrm{ft}^{2}{ }^{\circ} \mathrm{F}(2.16,1.15$, and $0.064 \mathrm{~W} / \mathrm{m} \cdot \mathrm{C})$, respectively; were used in these calculations.

The heat. losses predicted by the finite element and the finite difference computer programs for the carrier pipes installed in various concrete trench systems for hot water and steam distributions are shown in Table 2 . In general, for the heat supply pipes the calculated heat loss values from the finite element model are consistently about 6 percent smaller cornared to the results of the thermal analyzer. The differences in heat loss values tend to be greatest for the largest pipe diameter. With the exception of one case involving a 12 in. $(305 \mathrm{~mm})$ pipe carrying hot water, the finite element model used in this study gives approximately 11 percent greater heat loss values for the return pipes in comparison to the finite difference technique. The total pipe heat losses predicted by the finite element program agree within 4 percent from the thermal analyzer predictions. 
The effect of trench size on pipe heat losses using the finite elenent computer program was studied by systematically varying the inner dimension of the concrete trench containing the two 6-in. (152-mm) insulated pipes. The pipes are wrapped in a 3 in. $(76.2 \mathrm{~mm})$ thick insulation and aluminum jacket, and transport hot water at $350 \mathrm{~F}(177 \mathrm{C})$ and $285 \mathrm{~F}(141 \mathrm{C})$, respectively. The centers of these pipes are located at $2.75 \mathrm{ft}(0.34 \mathrm{~m})$ and $3.08 \mathrm{ft}(094$ m) below the ground surface and are separated by a horizontal distance of 1.90 ft $(0.58 \mathrm{~m})$. The results of this parameter analysis are shown in Table 3 . The trench dimension has little effect on pipe heat loss predictions as illustrated in the table. For example, doubling the trench dimensions from $3 \times 3 \mathrm{ft}(0.91 \times 0.91 \mathrm{~m})$ to $6 \times 6 \mathrm{ft}(1.83 \times 1.83 \mathrm{~m})$, causes only a $1.5 \%$ increase for the pipe heat loss.

4. Life-Cycle Cost

The total life-cycle cost of an underground heat distribution system is the sum of the cost of acquisition, yearly maintenance and repair cost, and yearly fuel energy cost including adjustment for future escalation in fuel prices over the life of the system. The total life-cycle cost (LCC) can be expressed as follows:

$\mathrm{LCC}=\mathrm{MIC}+8760 * Q * \mathrm{EC} \approx \mathrm{UPW}+\mathrm{MRC}$

where MIC = the material and installation costs, ( $\$ / f t$ of the underground system)

$Q=$ the system heat loss rates of two pipes, $(\mathrm{Btu} / \mathrm{h} \cdot \mathrm{ft})$; and 8760 is a conversion Eactor

$E C=$ the equivalent fuel energy cost, $(\$ / M B t u)$ 
$=100(\mathrm{FC} / \mathrm{EF})$, in which FC is fuel cost, $(\$ / \mathrm{MBtu})$, and $\mathrm{EF}$ is $-\mathrm{plant}$ fuel conversion efficiency, (\%)

UPW = the modified uniform present worth factor adjusted for future escalation in fuel prices

MRC = the maintenance and repair costs, ( $\$ / f t$ of the system)

The concrete trench had different overall dimensions to accomodate yarious pipe sizes and insulation thicknesses and to maintain specified clearances such as at least 4 inches $(102 \mathrm{~mm})$ of space existing between the insulated pipes, a minimum of 3 inches $(76 \mathrm{~mm}$ ) between the piping and ad joining trench walls, and at least 6 inches $(152 \mathrm{~mm}$ ) from the bottom surface of the pipe to the trench floor as specified in the design guide [8] and shown in Figure 1. A-minimum inside width of $2 \mathrm{ft}(0.61 \mathrm{~m})$ was used for all concrete tremches since the trenches smaller than this were difficult to work with during the stage of construction. In district heating and cooling, high temperature hot water and pressurized steam are recognized as the most effective and economical working media. For hot water distribution in a twin pipe system, both the heat supply and the return lines generally have the sampipe:size. However, the return condensate line for steam distribution is: usuaily a smaller diameter pipe than the supply line due to the volume Eeduction of the steam condensing to hot water.

The cost of materials and labor employed for constructing sherllow trench heat distribution systems of various pipe sizes and trench dimensions can be calculated using the cost data and estimate procedures presented in references $[2,3,9,10]$. Construction costs for shallow trench underground heat distribution systems are estimated and shown as a function of 
insulation thickness for nominal pipe sizes ranging from 1 to 18 inches (25 to $457 \mathrm{~mm}$ ) for hot water and steam distributions in Figures 3 to 25 . As illustrated in the figures, the construction costs for both the concrete trench and the insulated pipes increase with thicker pipe insulation. The estimated construction costs for some concrete trench and distribution piping systems were compared with those developed in the sample cases treated in the life-cycle cost analys is of underground heat distribution systems [2], and found to be in good agreement with those reported capital cost data.

The equivalent energy cost per unit length, or the yearly cost of lost energy considered as an operating expenditure, of the shallow trench system was calculated using the computed pipe heat losses obtained as the outputs from the computer program, and the fuel energy cost adjusted over the life of the system by taking projected energy price changes into account. The plant fuel conversion efficiency ranges between 50 and $90 \%$ depending on the type of boiler utilized. In all life-cycle cost calculations, the fuel to heat conversion efficiency at the plant is assumed to be $80 \%$.

The energy price is assumed to escalate annually in accordance with the data on the inflation rate of natural gas prices for the period 1985 to 2010 as projected by the U.S. Energy Information Administration [11]:

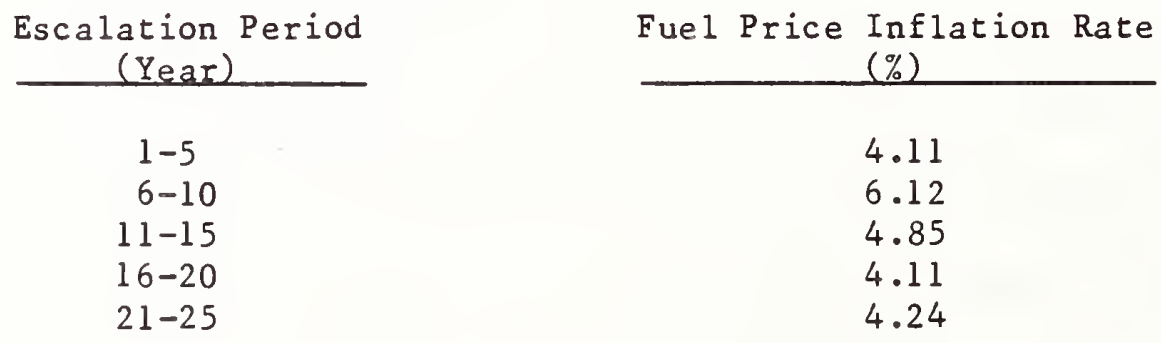


The modified uniform present worth factor (UPW) was calculated based on a $10 \%$ discount rate over a 25 year life for the underground shallow trench distribution system, and the fuel price escalation rate project by the U.S. Energy Information Administration for 1985 through 2010, using the following equation [12]:

$$
\begin{aligned}
\mathrm{UPW} & =\sum_{j=1}^{N_{1}}\left(\frac{1+e_{1}}{1+d}\right)^{j}+\left(\frac{1+e_{1}}{1+d}\right)^{N_{1}} \sum_{j=1}^{N_{2}}\left(\frac{1+e_{2}}{1+d}\right)^{j} \\
& +\left(\frac{1+e_{1}}{1+d}\right)^{N_{1}}\left(\frac{1+e_{2}}{1+d}\right)^{N_{2}} \sum_{j=1}^{N_{3}}\left(\frac{1+e_{3}}{1+d}\right)^{j} \\
& +\left(\frac{1+e_{1}}{1+d}\right)^{N_{1}}\left(\frac{1+e_{2}}{1+d}\right)^{N_{2}}\left(\frac{1+e_{3}}{1+d}\right)^{N_{3}} N_{4}\left(\frac{1+e_{4}}{1+d}\right)^{j} \\
& +\left(\frac{1+e_{1}}{1+d}\right)^{N_{1}}\left(\frac{1+e_{2}}{1+d}\right)^{N_{2}}\left(\frac{1+e_{3}}{1+d}\right)^{N_{3}}\left(\frac{1+e_{4}}{1+d}\right)^{N_{4}} \sum_{j=1}^{N_{5}}\left(\frac{1+e_{5}}{1+d}\right)^{j}
\end{aligned}
$$

where $\sum_{j=1}^{N_{k}}\left(\frac{1+e_{k}}{1+d}\right)^{j}=\left(\frac{1+e_{k}}{d-e_{k}}\right)\left[1-\left(\frac{1+e_{k}}{1+d}\right)^{N_{k}}\right]$

$\mathrm{N}_{k}=$ the length of the period for a given escalation rate in a given-period, (year)

$\mathrm{d}=$ the discount rate, $(\%)$

$e_{k}=$ the rate of escalation in each of $N_{k}$ period.

Assuming no salvage value for the underground shallow trench system at the end of a 25 year life-cycle, the modified uniform present worth factor, UPW is found to be $14.16 \%$.

Based on the cost data of a recent survey of field installations [1], the present values over the 25 year life-cycle of annually recurring routine 
maintenance and nonannully recurring repair costs for a typicai shailow trench system were computed and found to be $\$ 1.72$ per foot ( $\$ 5.64$ per meter) and $\$ 0.09$ per foot ( $\$ 0.30$ per meter), respectively. These maintenance and repaircosts were low compared to the systemconstructioncostand operational cost due to pipe heat losses and therefore neglected in I ife-cyclecost analysis.

For minimum life-cycle cost or maximum allowable heat loss calculations, the working fluid temperatures for the heat supply pipe were varied between 150 tand $500 \mathrm{~F}$ (66 to $260 \mathrm{C}$ ) with an increment of $50 \mathrm{~F}(27.8 \mathrm{C})$, and temperature levels of either $150 \mathrm{~F}(66 \mathrm{C})$ or $250 \mathrm{~F}(12 \mathrm{C})$ were selected for the return pipe fluid temperatures. Different pipe sizes ranging from 1 to 18 inches (25 to $457 \mathrm{~mm}$ ) in nominal diameter and insulation thicknesses varying from 1 to 6 inches ( 25 to $152 \mathrm{~mm}$ ) in increments of $0.5 \mathrm{in.}(12.7 \mathrm{~mm}$ ) were selected for heat loss calculations and cost analysis. In order to reflect a variety of fuel energy costs, the selected values of fuel cost ranged from $\$ 3.00$ to $\$ 15.00$ permilion Btu ( $\$ 2.84$ to $\$ 14.22$ per giga joule). All calculations were carried out based on an annual average earth temperature of $55 \mathrm{~F}$ ( 12.3 C), a soil thermal conductivity of $10 \mathrm{Btu} \cdot \mathrm{in} / \mathrm{h} \cdot \mathrm{ft} 2 . \mathrm{F}(1.44 \mathrm{~W} / \mathrm{m} . \mathrm{C}), a 6$ in. ( $152 \mathrm{~mm}$ ) thick trenchwall, a concrete thermal conductivity of $9.7 \mathrm{Btu} . \mathrm{in} / \mathrm{h} . \mathrm{ft} 2 . \mathrm{F}$ $(1.4 \mathrm{H} / \mathrm{m} \cdot \mathrm{C})$, an annual amplitude of the monthly average temperature cycle of $23 \mathrm{~F}(-5 \mathrm{C})[6,13]$, and heat losses determined for the month of January. The extreme seasonal temperatures were selected as the external temperatures for buried piping design considerations, and the choice of January resu. ted in the computed pipe heat loss to be approxinately $5 \%$ greater than annual heat loss. 
Computer calculations of the life-cycle cost for the cost of capital investment and the yearly energy cost associated with the heat lost from the pipes were carried out for each pipe size and specified fluid temperatuies, and for various insulation thicknesses starting from 1 in. ( $25 \mathrm{~mm}$ ) with an increment of $0.5 \mathrm{in.}(12.7 \mathrm{~mm})$. From a set of life-cycle costs derived for a range of insulation thickness, the maximum allowable heat losses for the supply and the return lines were selected as those heat losses for which the Iife-cycle cost was at a minimum for a specified fuel cost and pipe size. The pipe insulation thickness corresponding to these maximum allowable heat losses is the economic insulation thickness. This optimum insulaticit thickness is equivalent to the best economic balance between the total cost for constructing the shallow trench system and insulating the distribution piping, and the resulting energy saving over the life time of the underground system.

Tables A-1 through $\mathrm{C}-13$ present the naximum allowab le heat losses ( MAHL) and the corresponding economic insulation thickness (EIT) of the insulated pipes for shallow trench heat distribution systems at selected pipe temperatures and fuel cost. These tables are divided into three sets: the "A" tables present the MAHL and EIT values derived based on a $150 \mathrm{~F}(66 \mathrm{C})$ return pipe temperature for a high temperature hot water distribution system, the "B" tables contain MAHL and EIT data based on a $150 \mathrm{~F}$ (66 C) condensate return pipe temperature for a pressurized steam system, and the "C" tables present the MAHL and EIT values based on a $250 \mathrm{~F}(121 \mathrm{C})$ return pipe $\mathrm{f}$ uid temperature for a hot water distribution system. 
As iliustrated in the tables, the heat loss from the heat supply pipe increases as the temperature of the working medium flowing inside the supply

pipe increases, causing a larger temperature gradient between the inner and outer surfaces of the pipe insulation. However, the heat loss of the return pipe decreases as the supply pipe fluid temperature increases due to two factors; the increased insulation thickness and heat gainby convection heat transfer from the ad joining supply pipe. The effect of temperaturevariations of the return pipe on the maximum allowable heat losses can be determined by comparing the heat loss values in "A" tabie and those in "C" tavle for a given pipe size and fuel cost, and a selected working medium temperature of the supply pipe. The results indicate that a noticeable increase in the return pipe temperature does not cause any significant decrease in the heat loss value of the supply pipe. The heat loss of the return pipe at a temperature greater than $150 \mathrm{~F}(66 \mathrm{C})$ for a given pipe size, supply pipe fluid temperature and fuel cost can be found from one of the " $A$ " tables, and is equal to the heat loss value for the supply pipe at specific return pipe temperatuce. Using equation 1 and assuming a constant temperature for the outer surface of the insulation layer, this heat loss value is then revised to account for the reduction in pipe heat loss due to increased insulation thickness for specific return and supply pipe fluid temperatures. It should be noted that the maxinum allowable heat loss and the corresponding econonic insulation thickness data presented in the tables would change for different insulation materials, trench configurations and design conditions. 
5. Conclusions

The heat losses from two insulated pipes installed in a shallow trench underground heat distribution system vere calculated using a conpucer simulation program developed based on the application of the finite element method to two-dimensional, steady state heat conduction problem. A more accurate prediction of heat flows in an underground system and surrounding soilcanbe obtained us ing temperature-dependent, variable thermal conductivities for the pipe insulation. In general, the pipe heat losses predicted from the finite element model used in this study are in reasonably good agreement with the predictions of the finite difference computer model TASTAP. The effect of tzench dimension on pipe heat losses was studied numerically lising the finite element computer program and found to be insignificant. The material and labor costs for construction of concrete trenches distribution systems including insulated piping of various pipe sizes and insulation thicknesses, and concrete trench of different dimensions are develofed, and these cost data are presented graphically.

Life-cycle cost analysis was performed for shallow trench systems to obtain the pipe heat loss and the insulation optimumbalance between the costs of capital investment including the costs of constructing distribetion piping and installing additional insulation, and the resulting energy saving, calculated over the expected life of the underground system for projected growth of the fuel price. The procedures for calculating the energy cost attributed to the pipe heat loss and fordetermining the maximumallowab le heat loss, which is defined as that heat loss rate for which the total owning and operating cost of the system is at a minimum, for an underground concrete 
trench system are described. Based on the resuits of the cost analysis, the maximum aliowable heat losses and the most economic insuiation thichness for underzrolnu pipes distributing stean and hot water, were determined and tabulated for a wane ô fluid temperatures and pipe sizes, various levels of fuel cost, for fixẻd values of earth temperature and soil thermal conductivity.

6. Acknowledgment

This investigation was conducted under the Tri-Service Building Materiais Investigaticra: Program and was jointiy sponsored by the Headquarters, U.S. Army Corps of Enerineers; U.S. Navy, Naval Facilities Engineering Command; and the U.S. Air Force, Air Force Engineering and Services Center. The author would like to thank Sandra krause of NBS for her help in preparing the figures used in this report, and Robin Bickel or NBS for her skillful typing of the manucript of this reporting including numerous tables。

7. References

1. Pan Am World Services, Inc., "Heat Distribution Systems Life-Cycle Cost AnalysisComparison Between Direct Buried and Shallow Trench Systems," Report 130319, July 1985.

2. Parsons Corforation, "Life-Cycle Cost Anaiysis - Comparison Eetween Direct Euried Conduit and Shaliow Concrete Trench Underground Heat Distribution Systerns," February 1986.

3. Kusuda, T., "Maximum Allowable Heat Losses for Underground Feat Distribution Systems Based Upon Life-Cycle Cost Analysis," a report for Tri-Service Comnittee or Underground Heat Distribution Systems, April 1984.

4. U. S. Army Corps of Engineers, Guide Specification Military Construction, Underground Heat Distribution System and Condensate 
Return System - Prefabricated or Pre-Engineered Types', CEGS-15705, May 1986.

5. Kusuda, T., "FEvitke - Einite Element Method Computer Probran for Underground Heat Distribution System Heat Loss Calculations," a report. for Tri-Service Committee on Underground Heat Distribution Systens, January 1984.

6. Fang, J. B., "Minimum Life-Cycle Cost Heat Losses for Shallow Trench Underground Heat Distribution Systems," NBSIR 86-3381, May 1986.

7. U.S. Department of Energy, 'Economic Thickness for Industrial Insuiation,' The Fairmont Press Inc., Atlanta, GA, 1983.

8. U.S. Army Corps of Engineers, Guide Specification Military Construction, - Heat Bistribution Systems Outside of Buildings - Concrete Shallow Trench Systems, CEGS - 15709, June 1986.

9. Means Mechanical Cost Data, 7 th Annual Edition, Robert Snow Mleans Co., Kingston, MA, 1984 .

10. Building Construction Cost Data 1985, 43 rd Annual Edition, Robert Snow Means Co., Kingston, MA, 1985.

11. Lippiatt, B. C., Weber, S. F. and Ruegg, R. T., "Energy Prices and Discount Factors for Life-Cycle Cost Analysis," NBSIR 85-3273, November 1985.

12. Ruegg, R. T., "Life-Cycle Costing Manual for the Federal Energy Management Programs," National Bureau of Standards Handbook 135 , (Rev.), May 1982 .

13. Kusuda, T. and Achenbach, P. R., "Earth Temperature and Theimal Diffusivity at Selected Stations in the United States," ASHRAEIransactions, Vo1. 71, Part 1, Pp. 61-75, 1965. 
A Comparison of the Pipe Heat Losses Calculated from Temperature Dependent Thermal Conductivity with Those Obtained from Constant Thermal Conductivity of Calcium Silicate Insulation

Insulation Thickness (inch)

$$
1.0
$$

1.5

2.0

2.5

3.0

3.5

4.0

5.0

6.0
Heat

Constant K Temp. D

\section{Temp}

Pipe 1 Pipe 2 Tota

238

94

332

251

206

178

157

142

130

114

102
251

187

151

129

114

103

94

82

73
32

70

35

\section{Dep}

Pipe

Diff.

$(8)$

3.8

3.5

62

213

3.3

54

183

2.7

48

162

3.1

43

146

2.7

40

134

3.0

35

117

2.6

32

105

2.9 
Comparisons of Pipe Heat Loss Values Calculated by Finite Element Computer Program with Those by the Thermal Analyzer Program for Various Concrete Trench Systems

1. High temperature hot water systems with pipe temperatures of $350 \mathrm{~F}$ and $285 \mathrm{~F}$ :

$\begin{array}{lccc}\begin{array}{l}\text { Nominal Pipe } \\ \text { Diameter (Inch) }\end{array} & 1 & 6 & 12 \\ \begin{array}{l}\text { Insulation Thickness } \\ \text { Inch) }\end{array} & 2 & 3 & 3 \\ \text { Heat Loss (Btu/h.ft) } & & & \\ \text { Supply Pipe (350 F) } & 46.57(47.30) & 96.84(97.00) & 154.06(171.97) \\ \text { Return Pipe (285 F) } & 35.85(34.75) & 73.74(71.69) & 115.61(126.80) \\ \text { Total } & 82.42(82.05) & 170.58(168.69) & 269.67(298.77)\end{array}$

2. Pressuriżed steam systems with pipe temperatures of $350 \mathrm{~F}$ and $160 \mathrm{~F}$ :

Nominal Pipe

Diameter (Inch)

Supply Pipe 126

12

Return Pipe $3 / 4$ 3

6

Insulation Thickness

(Inch)

Supply Pipe

Return Pipe

Heat Loss $(B t u / h \cdot f t)$

Supply Pipe (350 F) $47.05(49.68)$

Return Pipe (160 F) $14.08(10.94)$

Total
$61.13(60.62)$
$98.97(101.68)$

$159.98(181.28)$

$23.10(19.16)$ $29.01(25.01)$ $122.07(120.84)$
3

3

Note: The pipe heat loss values in brackets are predicted by the finite difference thermal analyzer program TASTAP. 
TABLE 3

Effect of Trench Dimension on Pipe Heat Losses

Concrete Trench Inner Dimension (width $x$ height)

$\frac{\text { Pipe Heat Loss (Btu/h-ft) }}{\text { Supply Pipe Return Pipe }}$

$3 \mathrm{ft} \times 3 \mathrm{ft}$

97.02

73.35

170.37

$4 \mathrm{ft} \times 4 \mathrm{ft}$

96.84

73.74

170.58

$5 \mathrm{ft} \times 5 \mathrm{ft}$

97.19

74.08

171.27

$6 \mathrm{ft} \times 6 \mathrm{ft}$

97.57

74.44

172.01 


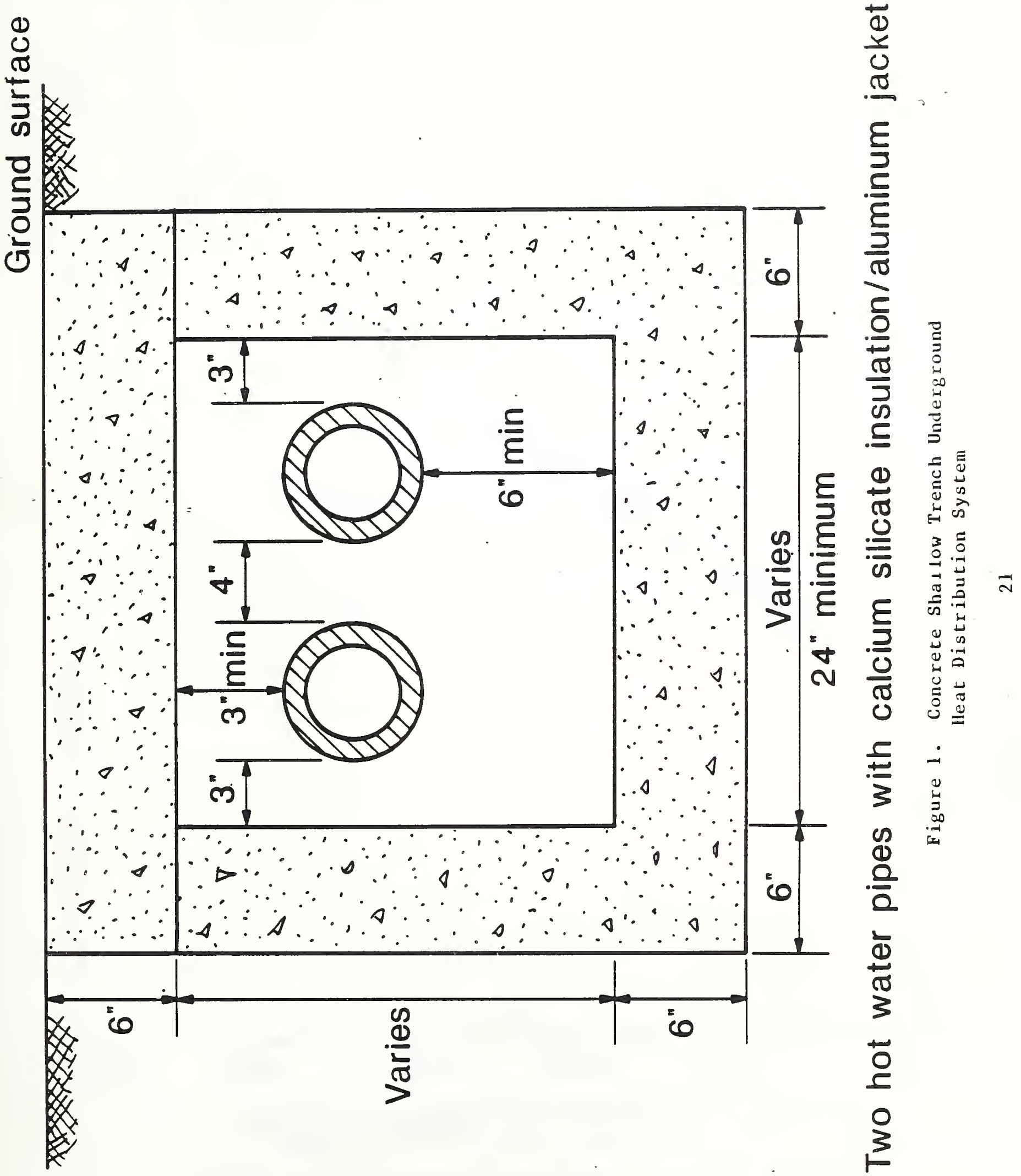




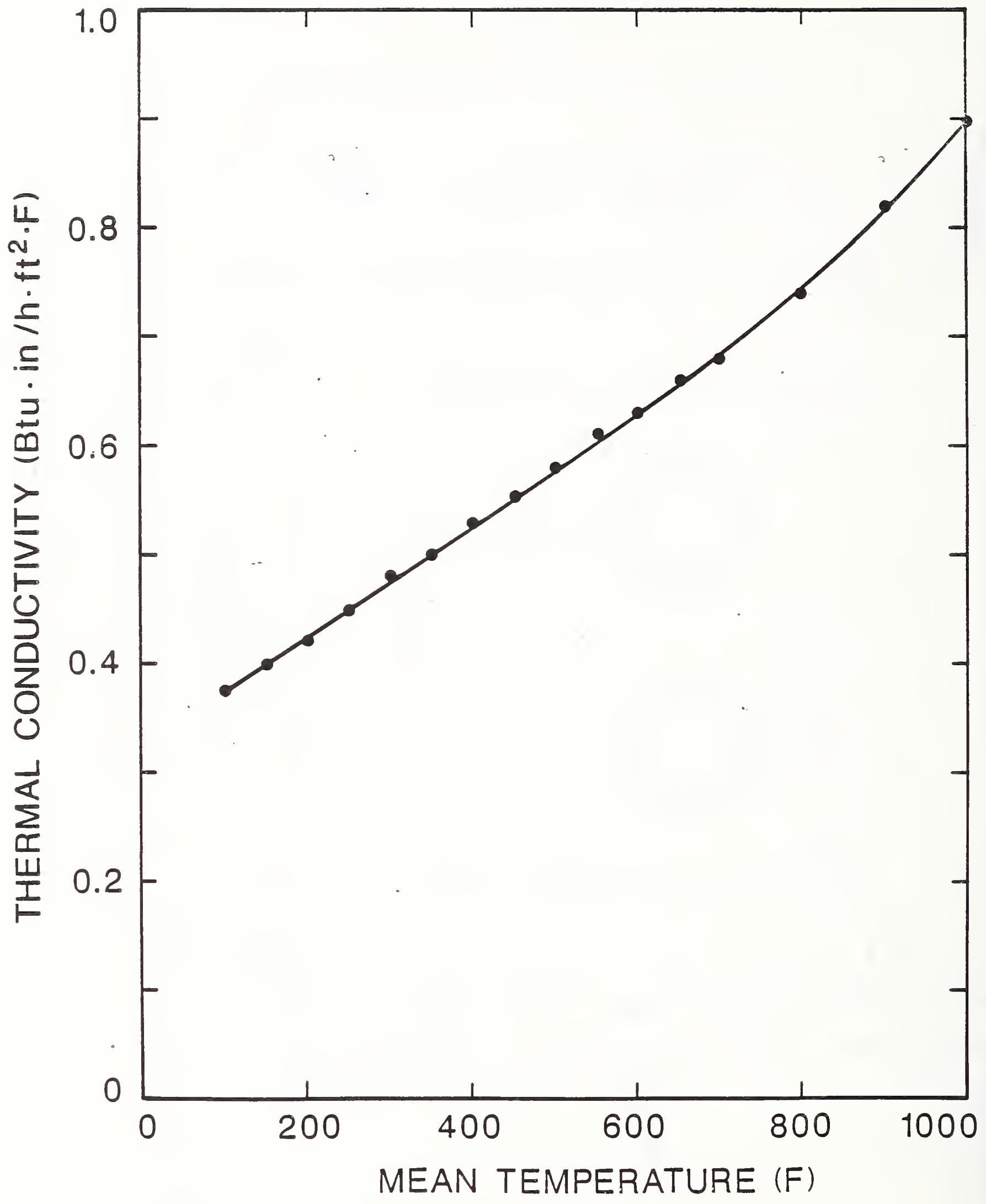

Figure 2. Thermal Conductivity Variation With Temperature for Calcium Silicate Insulation 


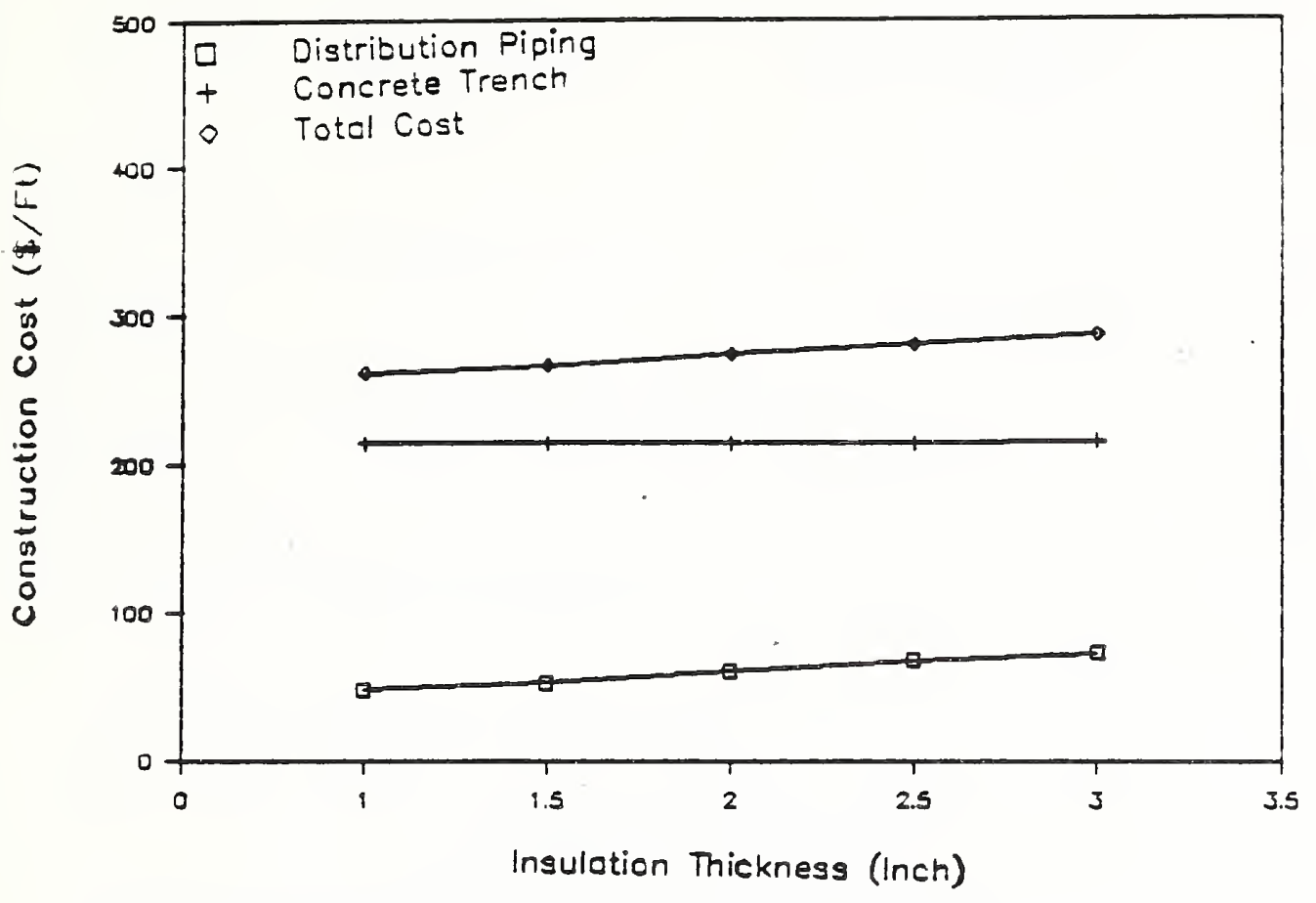

Figure 3. Construction Cost for a Shallow Trench System Containing Two 1 -inch Insulated Pipes



Figure 4. Construction Cost for a Shallow Trench System Containing Two 2-inch Insulated Pipes 


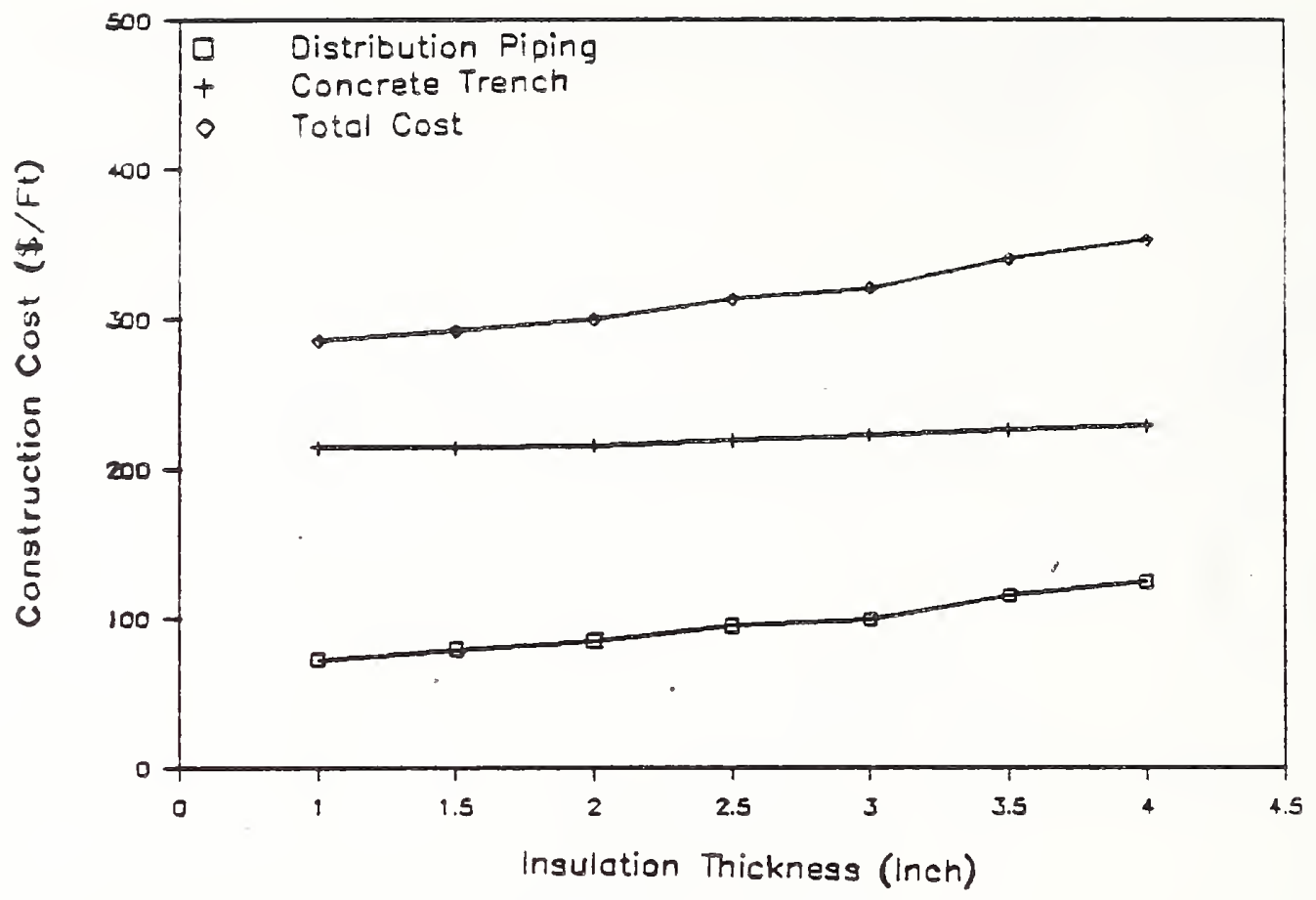

Figure 5. Construction Cost for a Shallow Trench System Containing Two 3-inch Insulated Pipes



Figure 6. Construction Cost for a Shallow Trench System Containing Two 4-inch Insulated Pipes 


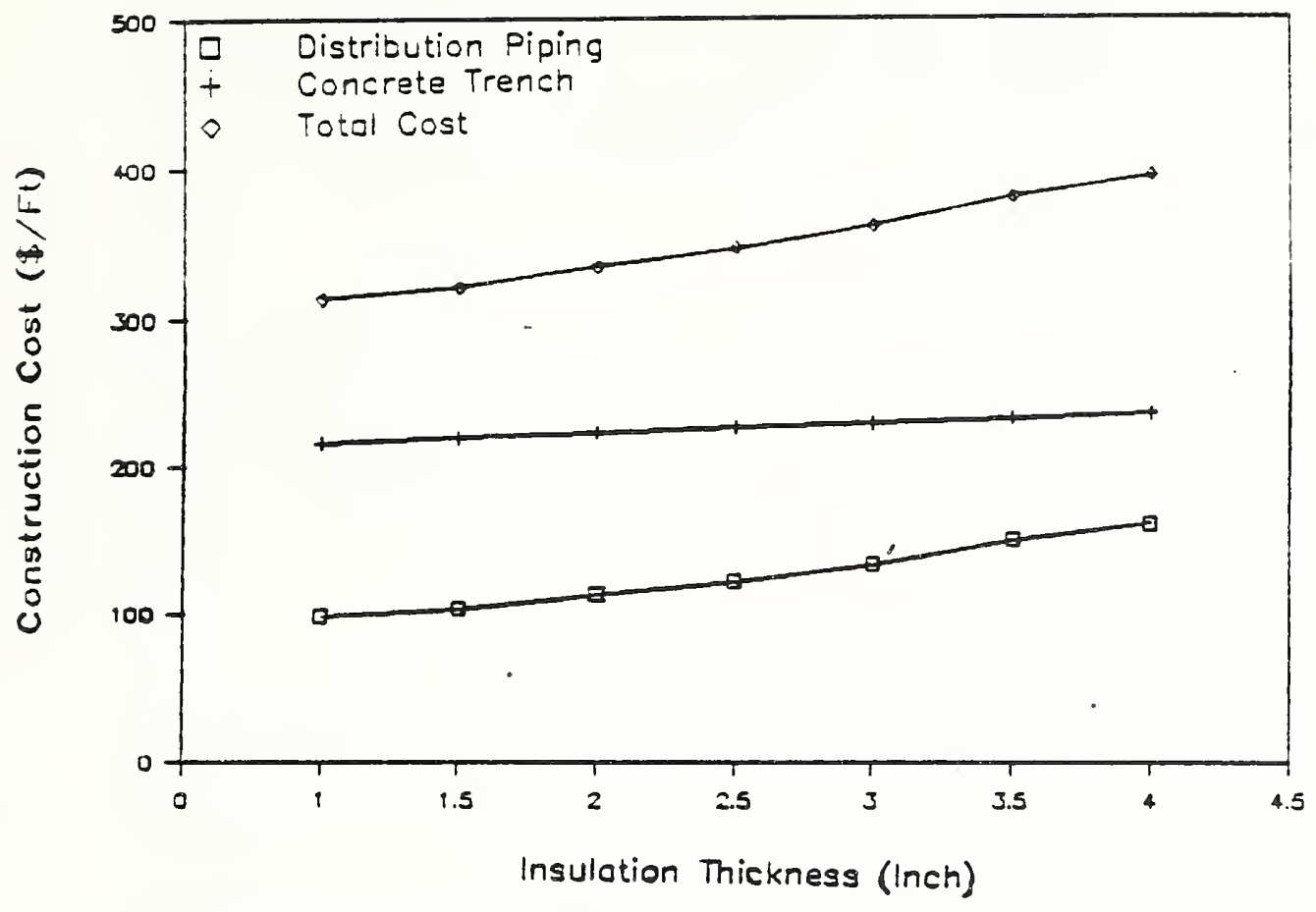

Figure 7. Construction Cost for a Shallow Trench System Containing Two 5-inch Insulated Pipes



Figure 8. Construction Cost for a Shallow Trench System Containing Two 6-inch Insulated Pipes 


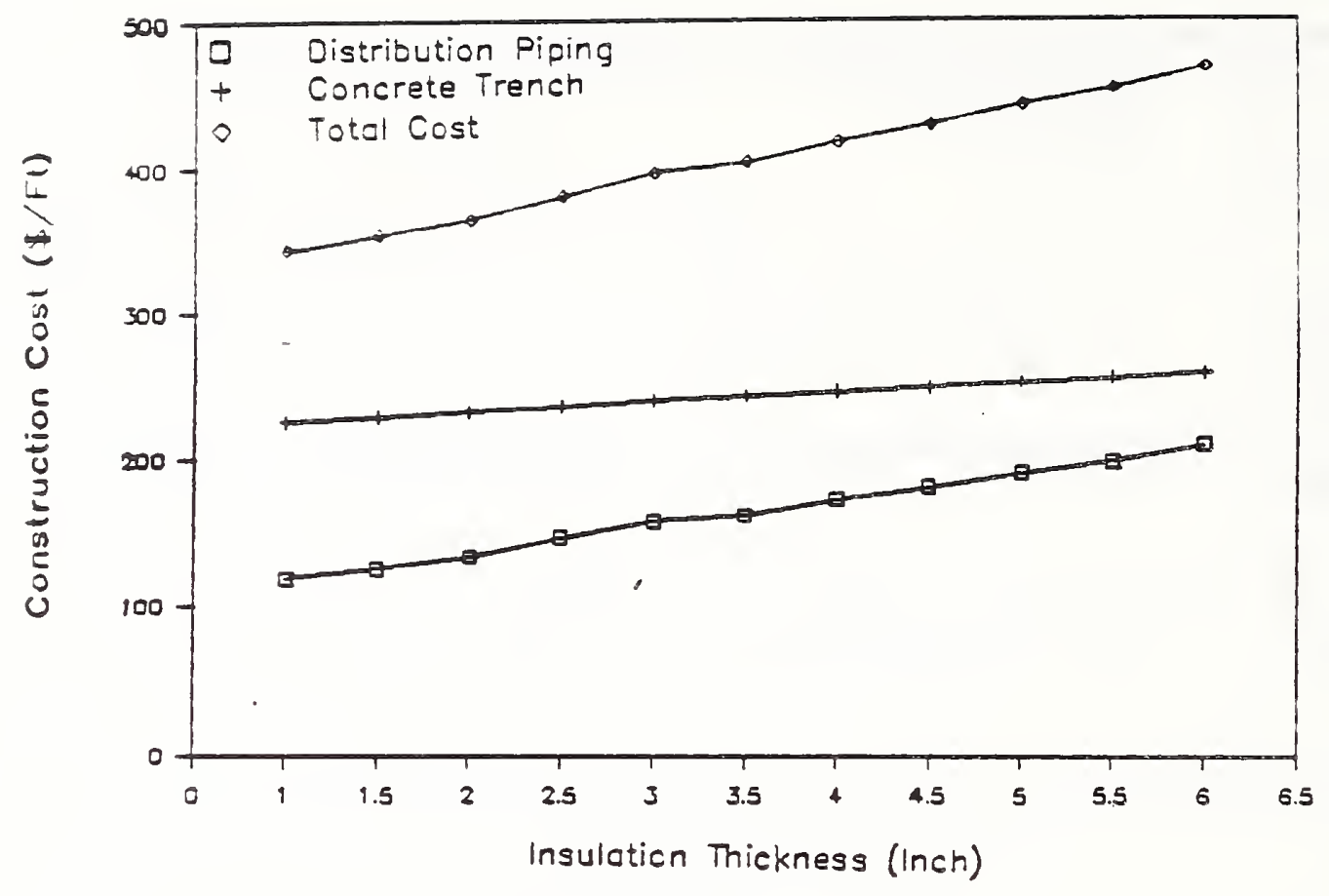

Figure 9. Construction Cost for a Shallow Trench System Containing Two 8-inch Insulated Pipes

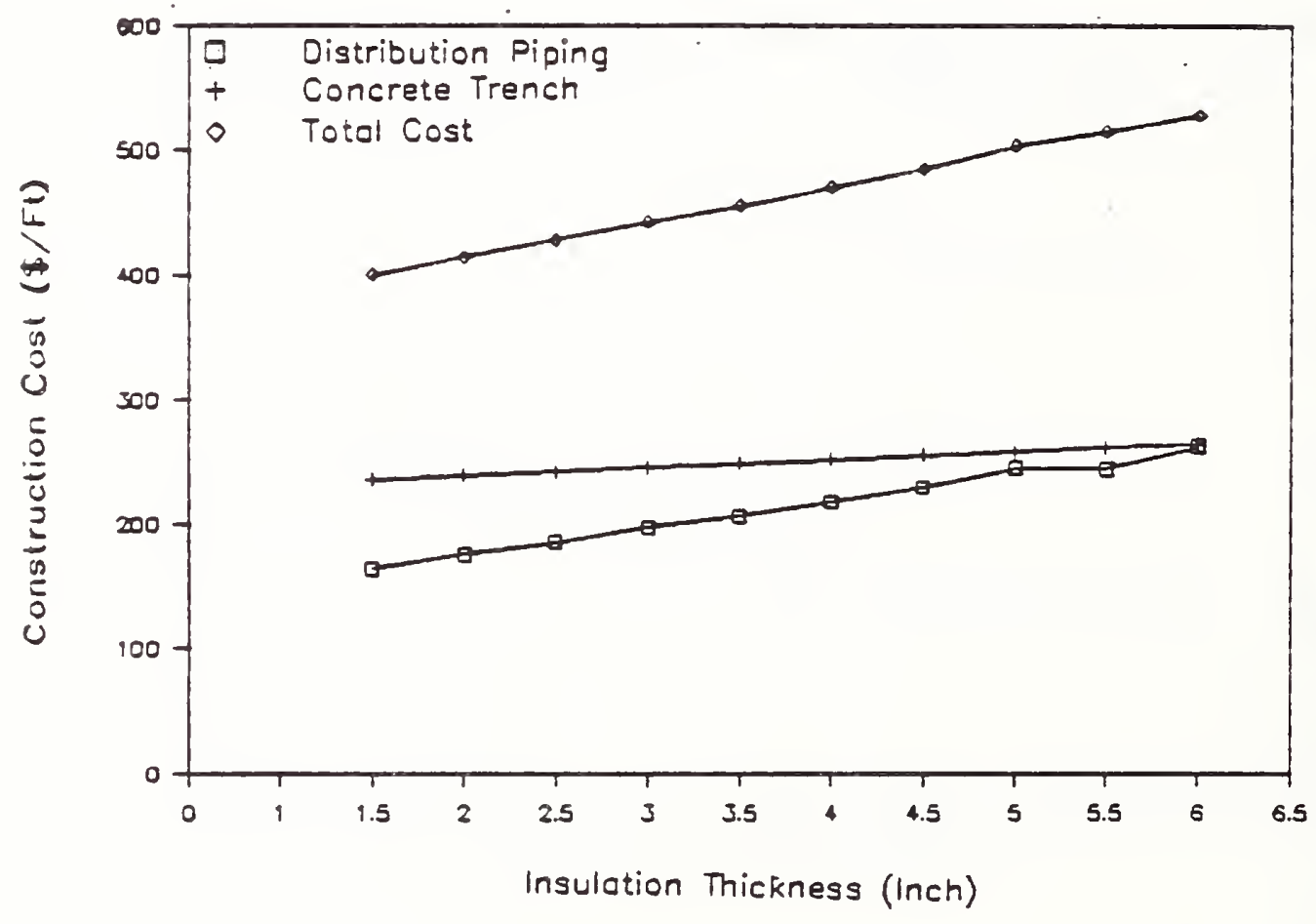

Eigure 10. Construction Cost for a Shallow Trench System Containing Two 10-inch Insulated Pipes 


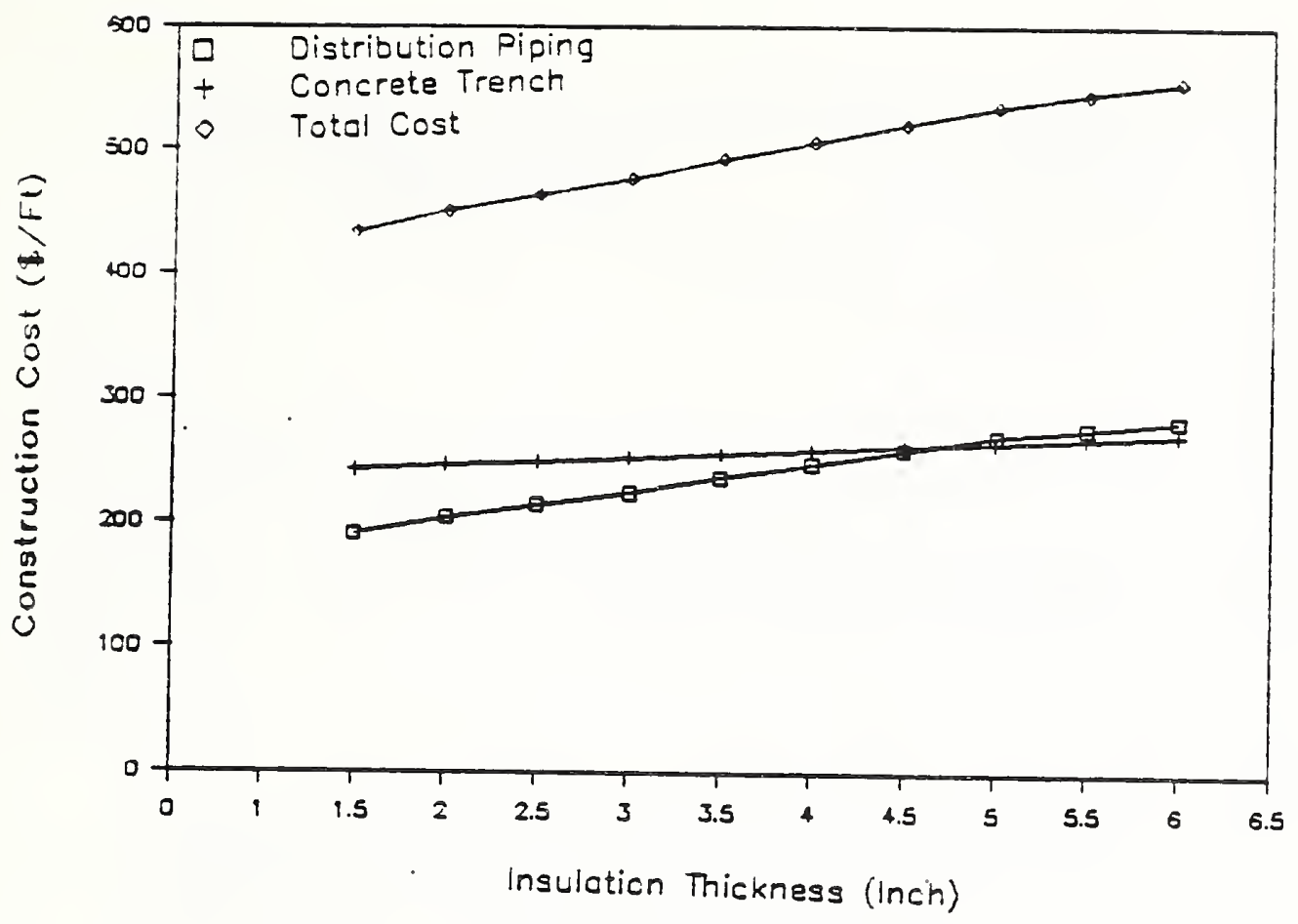

Figure 11. Construction Cost for a Shallow Trench System Containing Two 12-inch Insulated Pipes

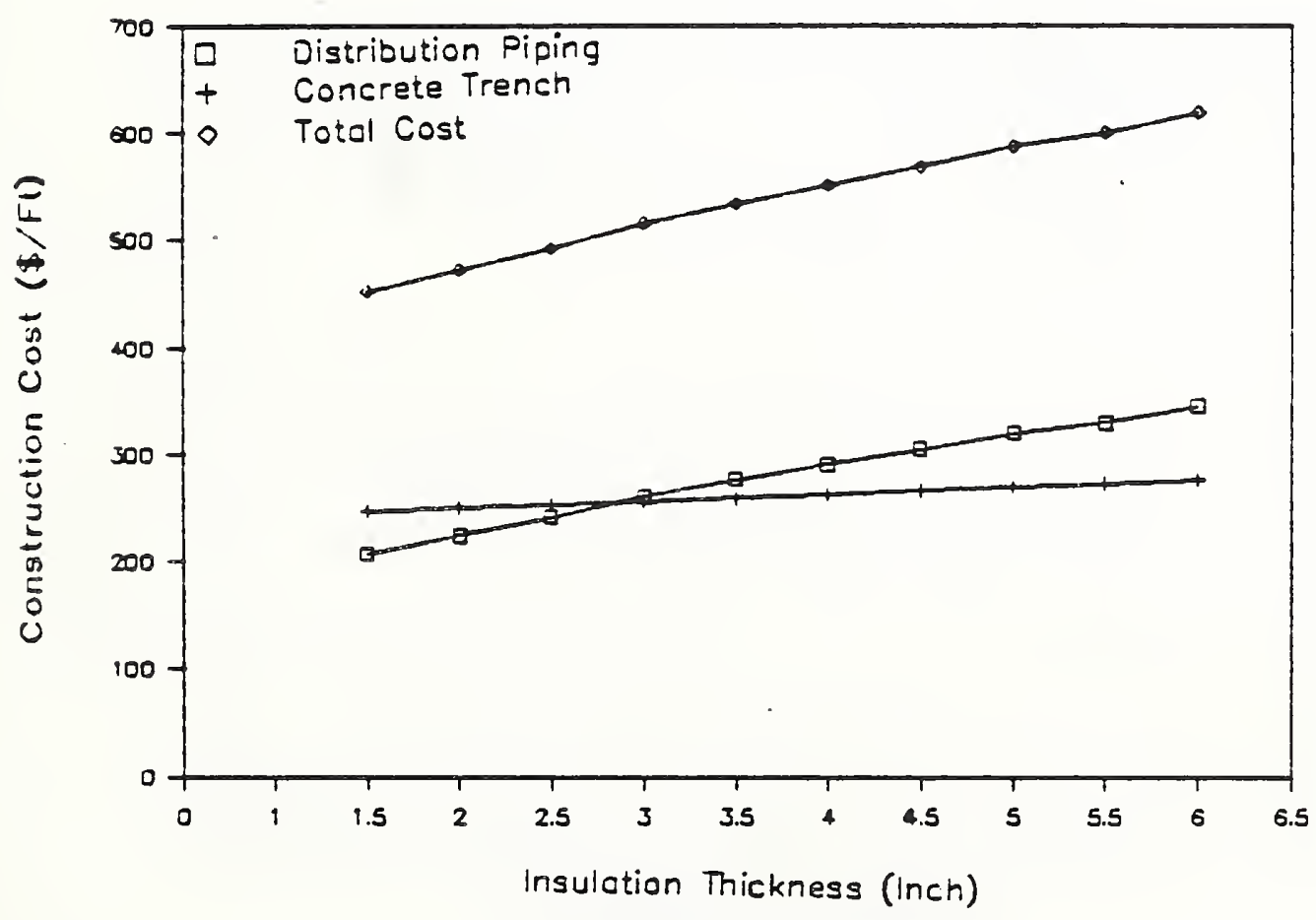

Figure 12. Construction Cost for a Shallow Trench System Containing Two 14-inch Insulated Pipes 




Figure 13. Construction Cost for a Shallow Trench System Containing Two 16-inch Insulated Pipes

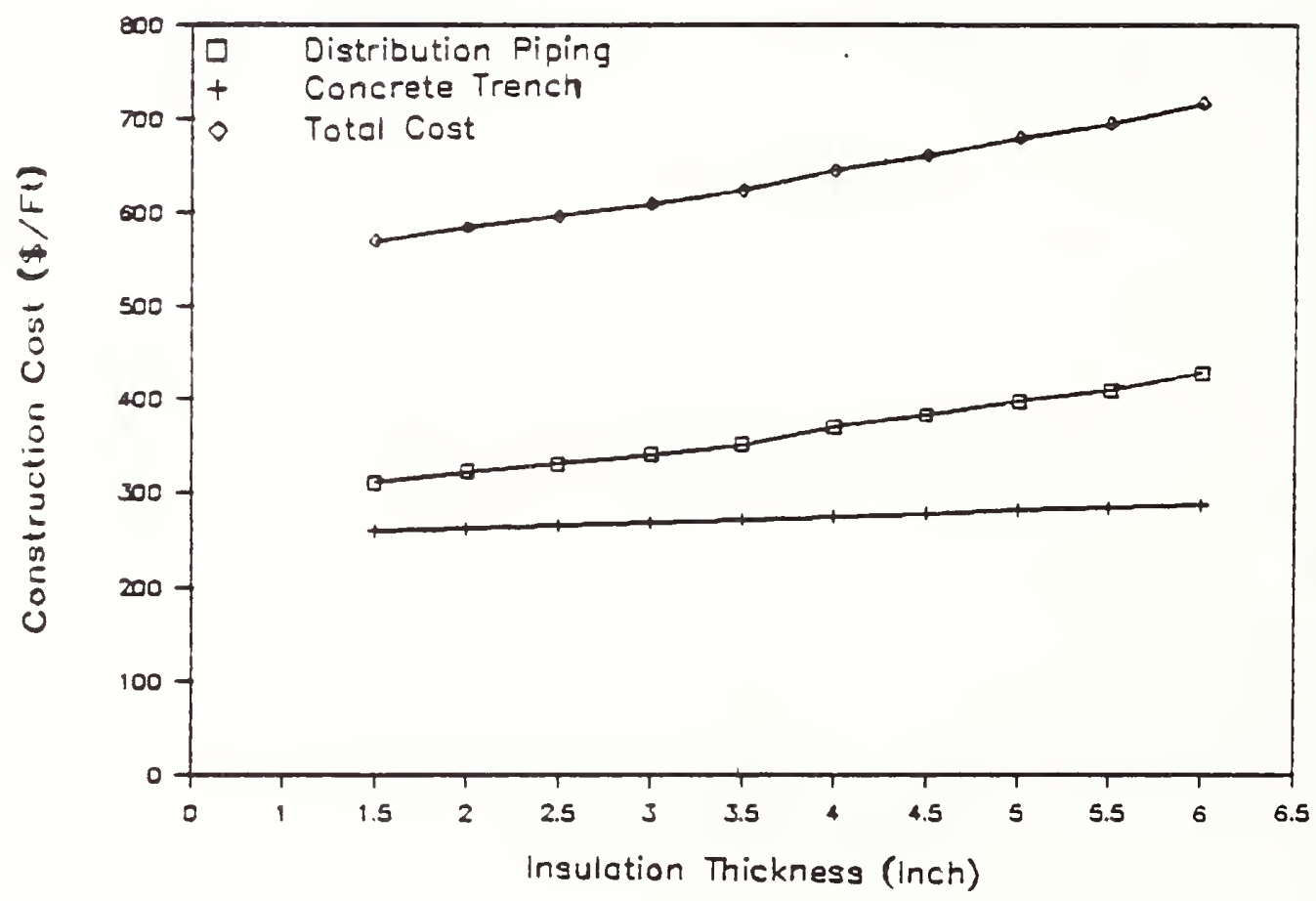

Figure 14. Construction Cost for a Shallow Trench System Containing Two 18-inch Insulated Pipes 


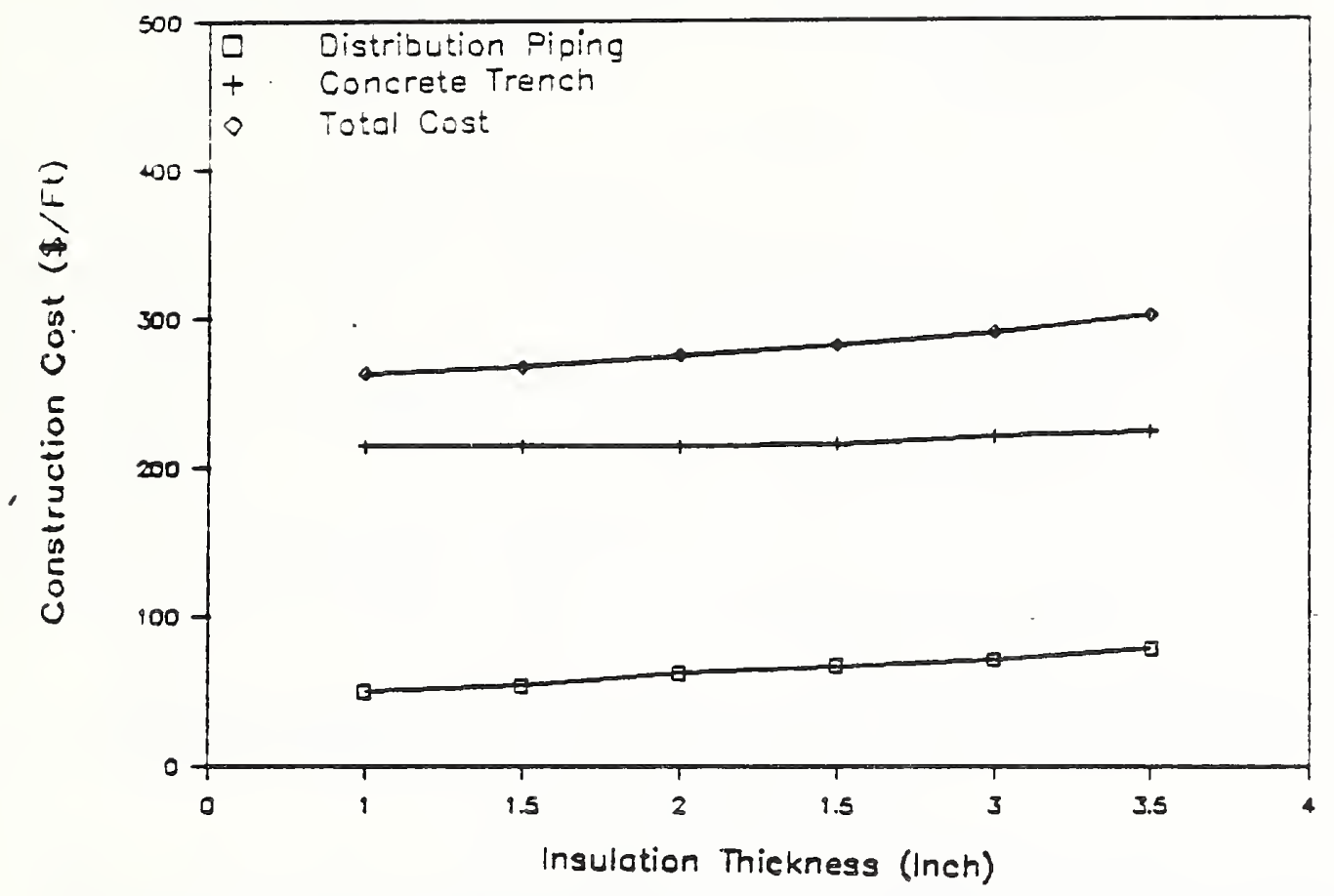

Figure 15. Construction Cost for a Shallow Trench System Containing a 2-inch Supply and a 1 -inch Return Insulated Pipes

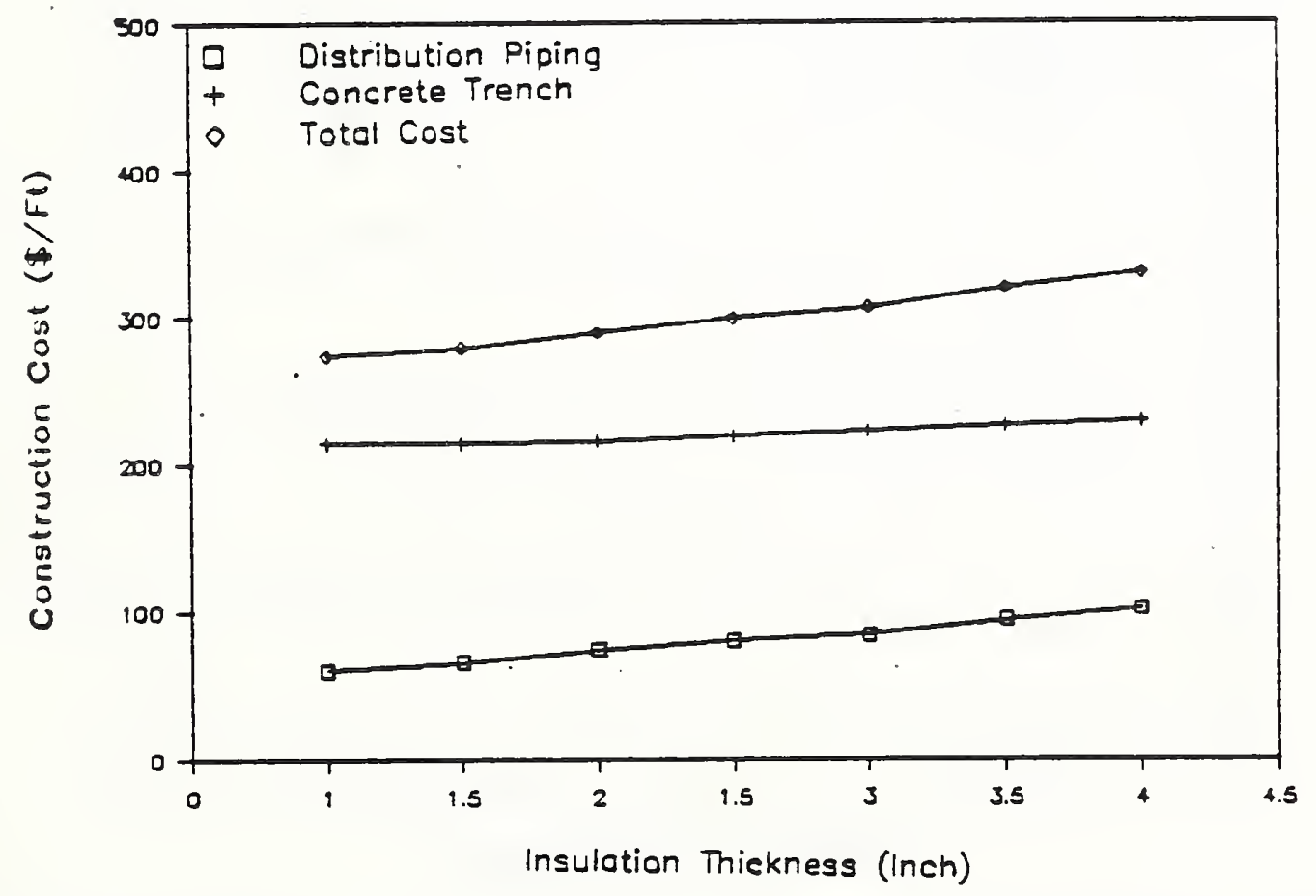

Eigure 16. Construction Cost for A Shallow Trench System Containing a 3-inch Supply and a 1.5-inch Return Insulated Pipes 


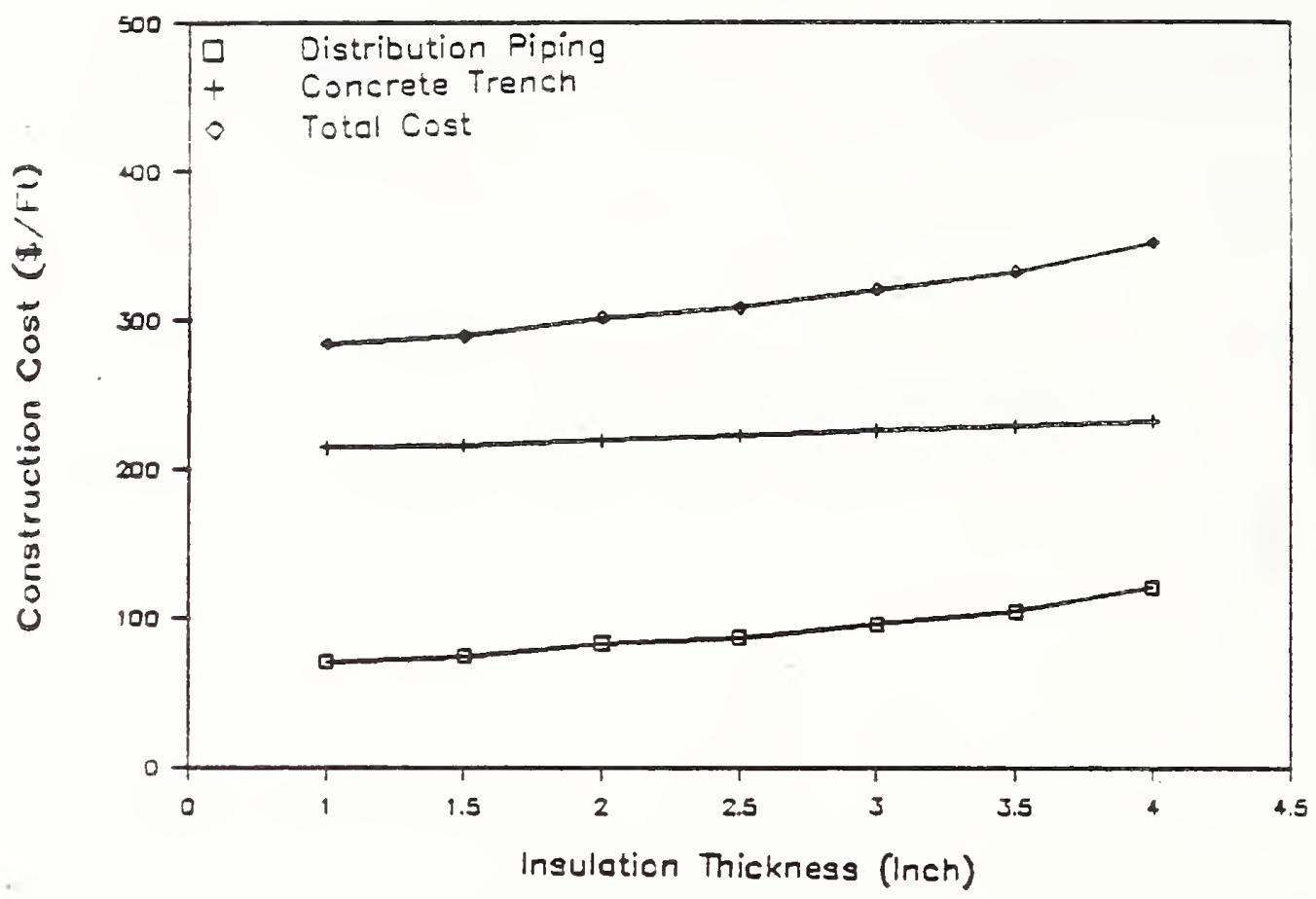

Eigure 17. Construction Cost of a Shallow Trench System Containing a 4-inch Supply and a 2-inch Rețurn Insulated Pipes



Figure 18. Construct Cost for a Shallow Trench System Containing a 5-inch Supply and a 2.5-inch Return Insulated Pipes 


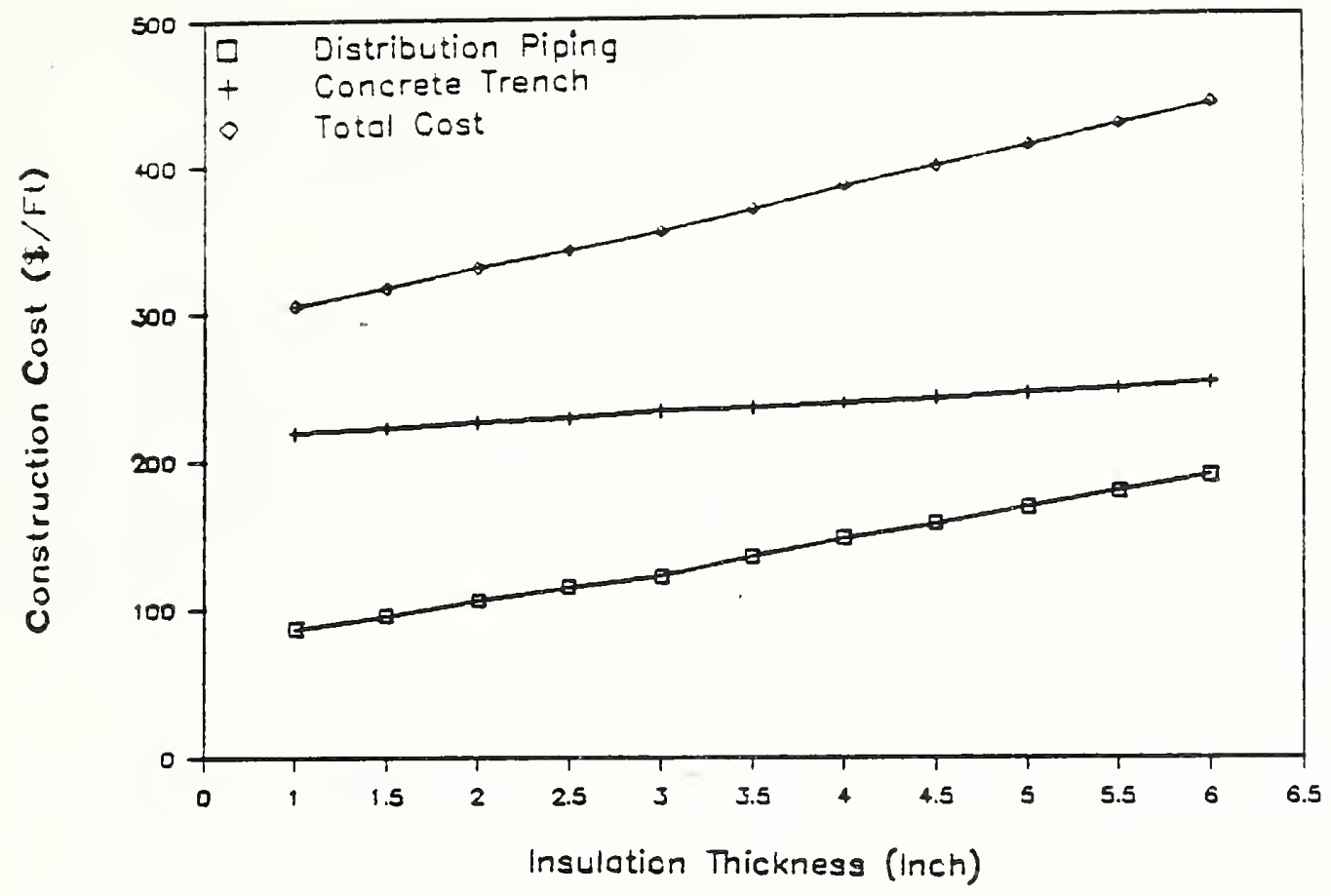

Figure 19. Construction Cost for a Shallow Trench System Containing a 6-inch Supply and a 3-inch Return Insulated Pipes

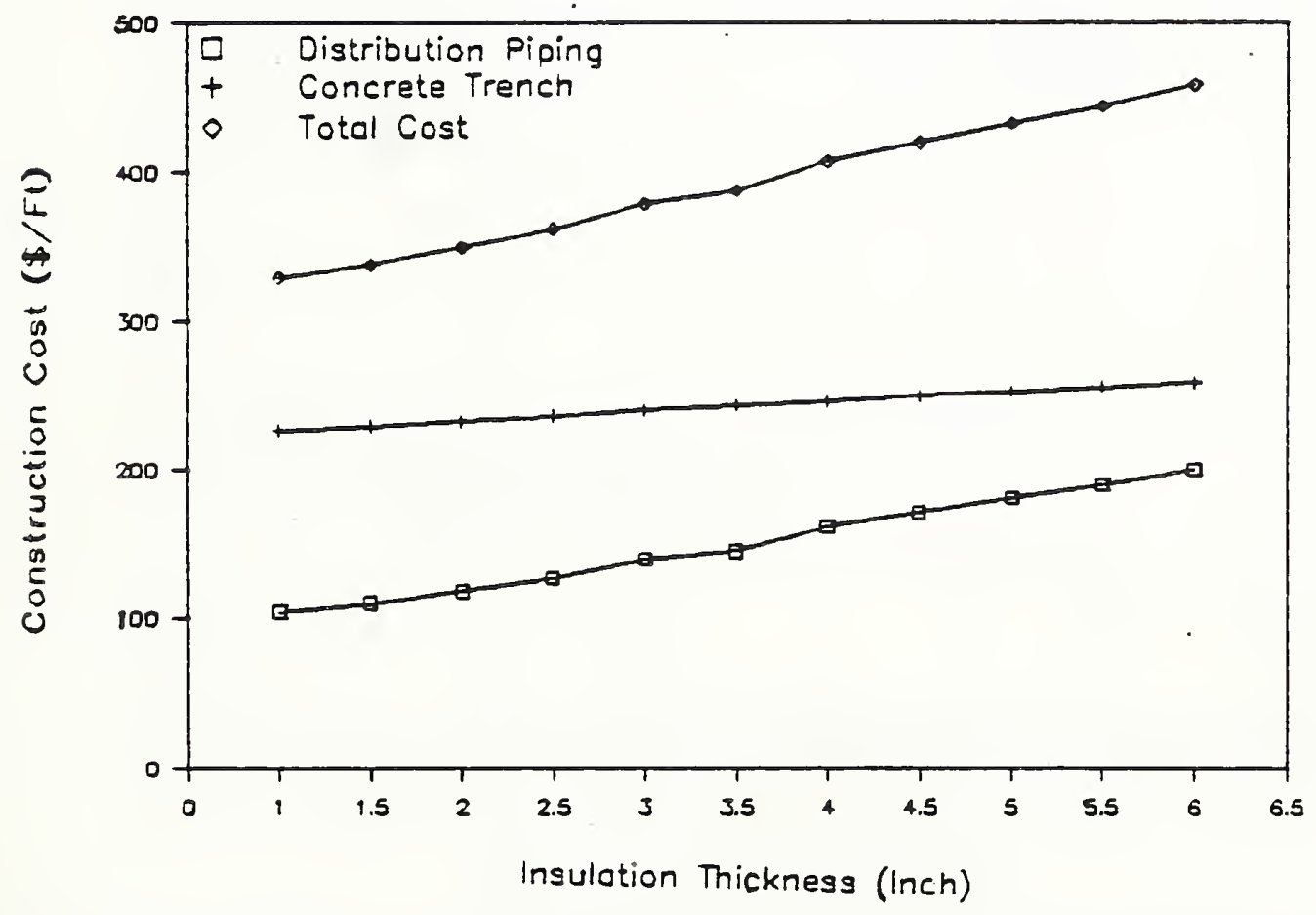

Figure 20. Construction Cost for a Shallow Trench System Containing a 8-inch Supply and a 4-inch Return Insulated Pipes 


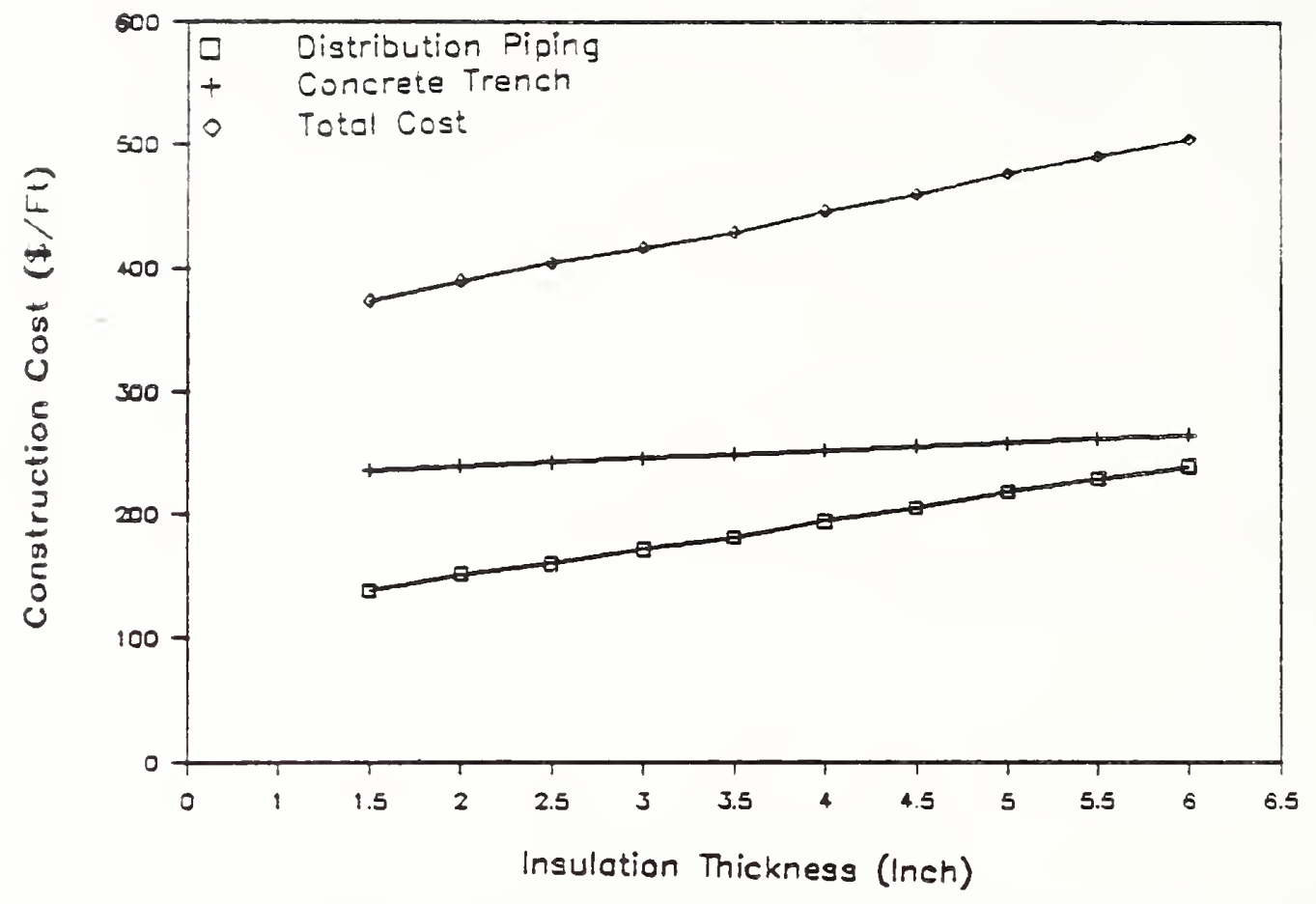

Figure 21. Construction Cost for a Shallow Trench System Containing a 10-inch Supply and a 6-inch Return Insulated Pipes

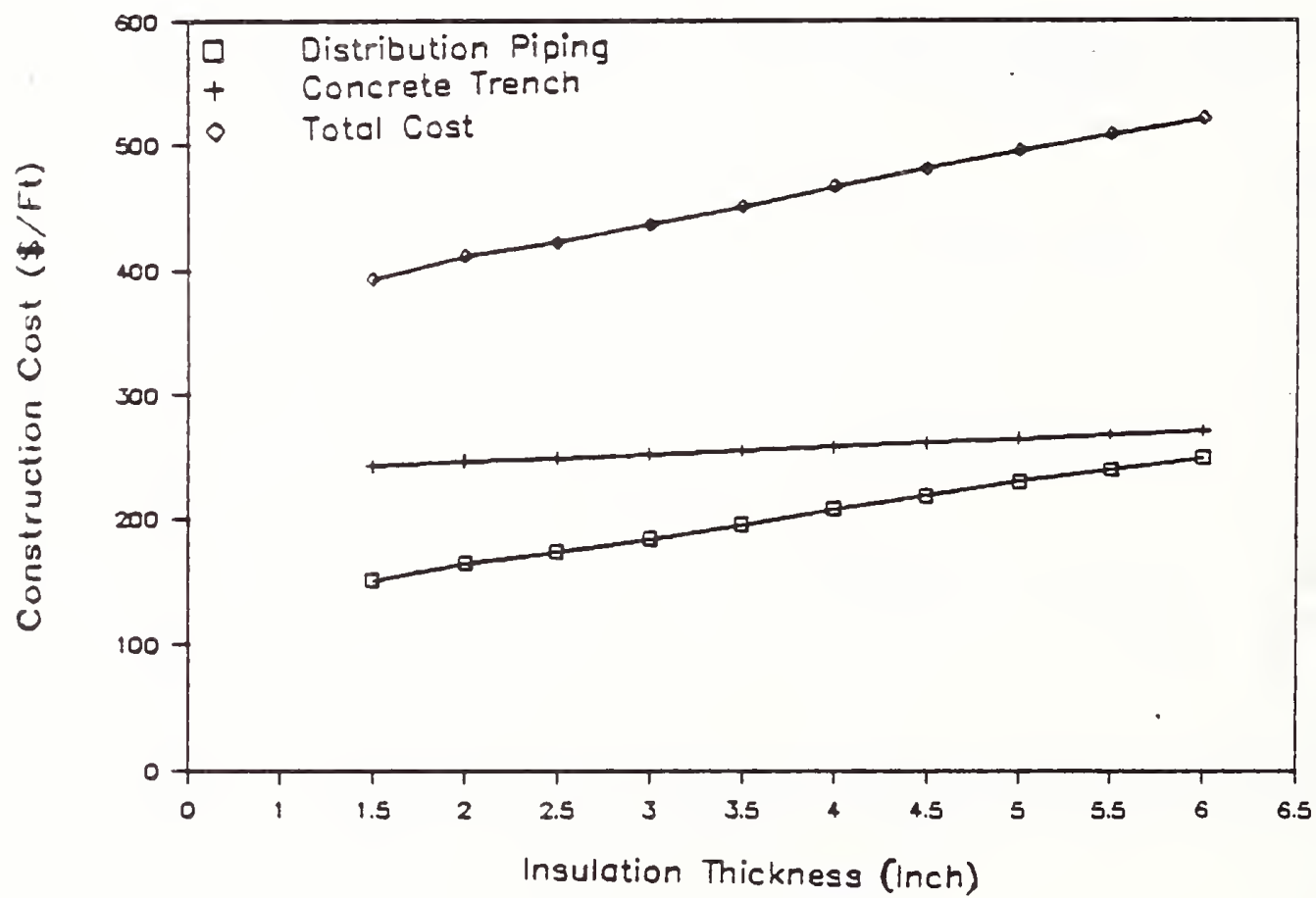

Figure 22. Construction Cost for a Shallow Trench System Containing a 12-inch Supply and a 6-inch Return Insulated Pipes 


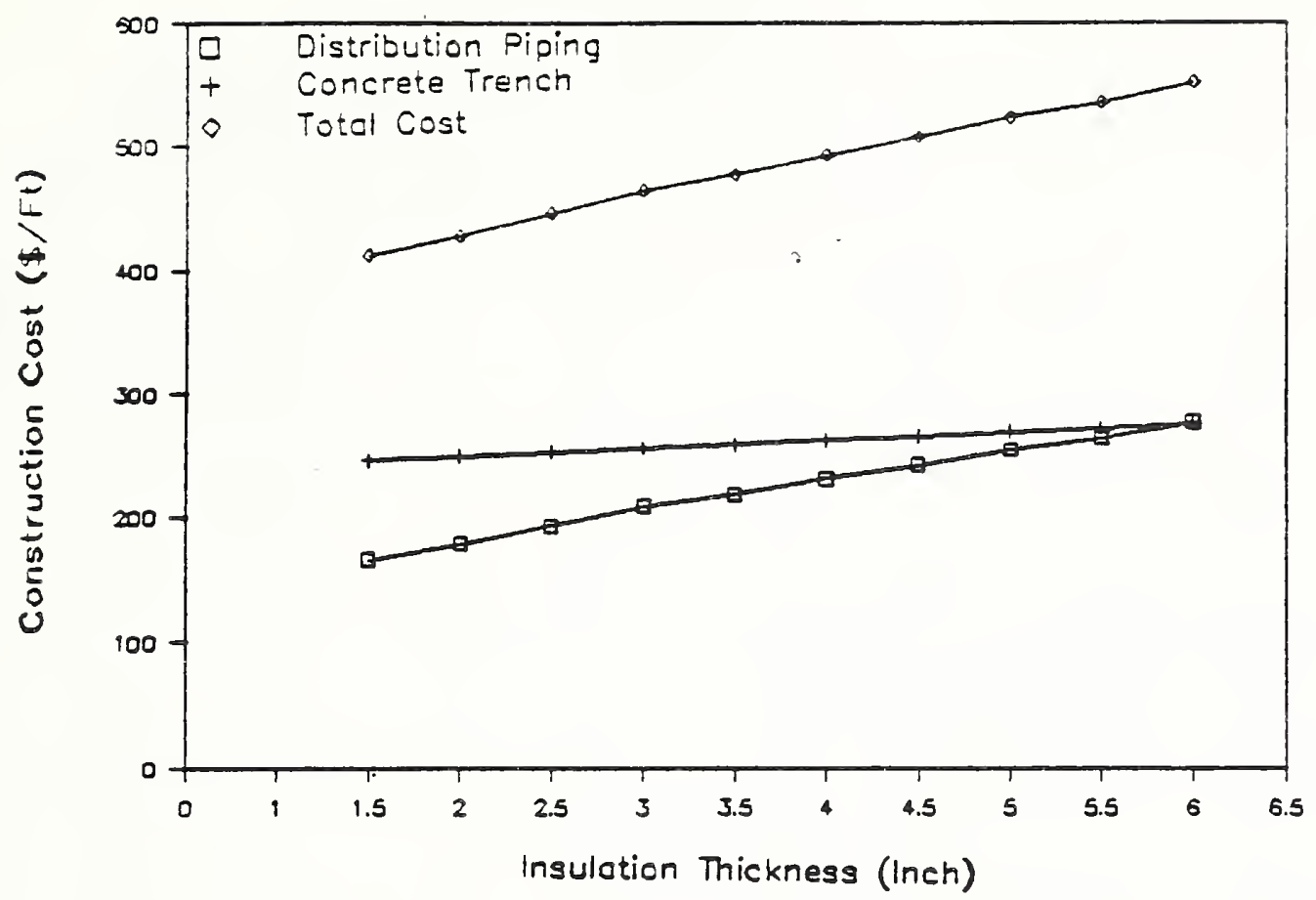

Figure 23. Construction Cost for a Shallow Trench System Containing a 14-inch Supply and a 8-inch Return Insulated Pipes

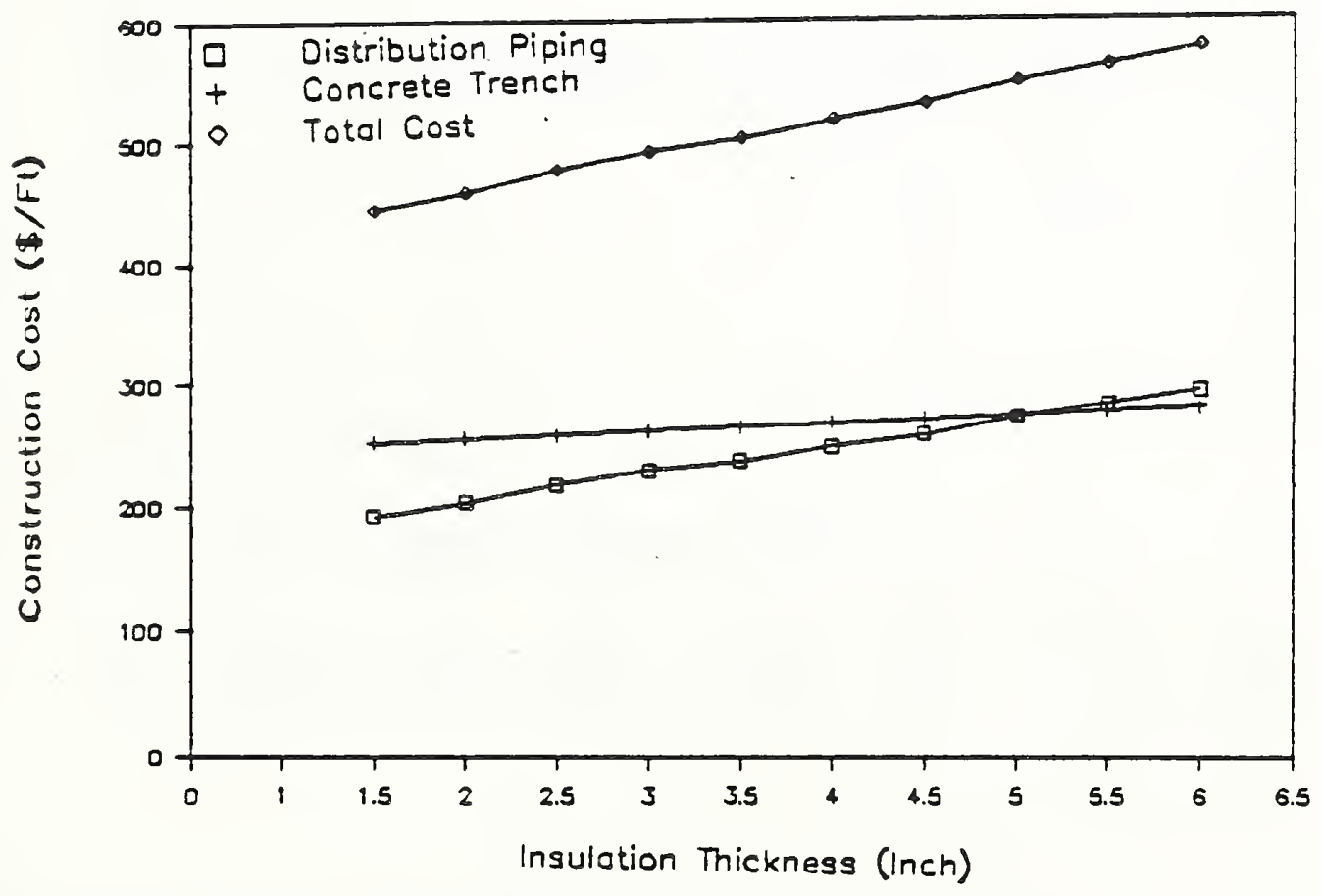

Figure 24. Construction Cost for a Shallow Trench System Containing a 16-inch Supplv and a 8-inch Return Insulated Pipes 




Figure 25. Construction Cost for a Shallow Trench System Containing a 18-inch Supply and a 10-inch Return Insulated Pipes 
TABLE A-1.a

Maximum Allowable Heat Losses (Btu/h. ft) from the Supply Pipe ( $S$ ) and the Return Pipe (R) for Energy Cost of $\$ 3 / M$ Btu

\begin{tabular}{|c|c|c|c|c|c|c|c|c|c|}
\hline \multirow{2}{*}{$\begin{array}{l}\text { Pipe } \\
\text { Size } \\
\text { (inch) }\end{array}$} & \multirow[b]{2}{*}{ Pipe } & \multicolumn{8}{|c|}{ Process Fluid Temperature (F) } \\
\hline & & $\underline{150}$ & $\underline{200}$ & $\underline{250}$ & 300 & $\underline{350}$ & 400 & 450 & $\underline{500}$ \\
\hline \multirow[t]{2}{*}{1} & $\mathrm{~S}$ & 22.2 & 26.2 & 35.3 & 45.1 & 55.6 & 66.6 & 77.8 & 89.5 \\
\hline & $\mathrm{R}$ & 22.2 & 17.3 & 17.1 & 16.8 & 16.5 & 16.2 & 15.9 & 15.6 \\
\hline \multirow[t]{2}{*}{2} & $\mathrm{~s}$ & 25.0 & 37.3 & 50.5 & 54.0 & 66.6 & 70.0 & 81.8 & 94.0 \\
\hline & $\mathbf{R}$ & 25.0 & 24.6 & 24.1 & 20.0 & 19.6 & 17.2 & 16.9 & 16.6 \\
\hline \multirow[t]{2}{*}{3} & S & 32.3 & 48.4 & 54.1 & 69.2 & 85.3 & 88.6 & 92.6 & 106.5 \\
\hline & $\mathrm{R}$ & 32.3 & 31.6 & 25.8 & 25.3 & 24.7 & 21.4 & 19.0 & 18.7 \\
\hline \multirow[t]{2}{*}{4} & $\mathrm{~S}$ & 38.5 & 57.8 & 64.2 & 70.7 & 87.3 & 104.6 & 108.6 & 124.9 \\
\hline & $\mathrm{R}$ & 38.5 & 37.4 & 30.4 & 26.0 & 25.4 & 24.8 & 22.0 & 21.5 \\
\hline \multirow[t]{2}{*}{5} & S & 44.8 & 67.5 & 74.6 & 81.8 & 101.0 & 121.0 & 125.0 & 144.0 \\
\hline & $\mathrm{R}$ & 44.8 & 43.5 & 35.0 & 29.8 & 29.0 & 28.3 & 25.0 & 24.4 \\
\hline \multirow[t]{2}{*}{6} & $S$ & 51.0 & 77.0 & 84.9 & 92.7 & 114.4 & 137.2 & 141.1 & 162.6 \\
\hline & $R$ & 51.0 & 49.3 & 39.5 & 33.4 & 32.5 & 31.6 & 27.8 & 27.0 \\
\hline \multirow[t]{2}{*}{8} & S & 62.1 & 76.4 & 103.6 & 112.8 & 139.2 & 146.2 & 153.1 & 176.4 \\
\hline & $\mathrm{R}$ & 62.1 & 49.2 & 47.7 & 40.0 & 38.8 & 33.8 & 30.2 & 29.4 \\
\hline \multirow[t]{2}{*}{10} & $S$ & 73.6 & 90.6 & 104.2 & 133.6 & 144.0 & 172.6 & 180.1 & 207.7 \\
\hline & $\mathrm{R}$ & 73.6 & 58.0 & 48.3 & 46.7 & 40.4 & 39.2 & 34.8 & 33.7 \\
\hline \multirow[t]{2}{*}{12} & $S$ & 84.0 & 103.6 & 119.1 & 152.8 & 164.3 & 197.1 & 204.8 & 236.3 \\
\hline & $\mathrm{R}$ & 84.0 & 66.0 & 54.8 & 52.8 & 45.6 & 44.0 & 38.8 & 37.4 \\
\hline \multirow[t]{2}{*}{14} & $S$ & 90.3 & 111.7 & 128.6 & 165.0 & 176.5 & 211.7 & 219.7 & 253.7 \\
\hline & $\mathrm{R}$ & 90.3 & 70.8 & 58.8 & 56.6 & 48.3 & 46.5 & 40.9 & 39.3 \\
\hline \multirow[t]{2}{*}{16} & $S$ & 100.9 & 123.9 & 142.0 & 158.4 & 195.5 & 208.2 & 243.3 & 253.6 \\
\hline & $\mathrm{R}$ & 100.9 & 95.5 & 64.1 & 54.6 & 52.5 & 45.9 & 44.2 & 39.4 \\
\hline \multirow[t]{2}{*}{18} & $S$ & 108.8 & 135.3 & 155.5 & 173.5 & 214.2 & 228.0 & 266.6 & 277.8 \\
\hline & $\mathrm{R}$ & 108.8 & $84: 5$ & 69.4 & 59.0 & 56.5 & 49.3 & 47.2 & 42.1 \\
\hline
\end{tabular}

Note: Energy Cost $=\$ 3.00 / \mathrm{MBtu}$

Return Pipe Temperature $=150 \mathrm{~F}$ 
TABLE $A-1 . b$

Economic Insulation Thickness (inch) for Hot Water Supply and Return Pipes for Energy Cost of $\$ 3 / \mathrm{MBtu}$

\begin{tabular}{|c|c|c|c|c|c|c|c|c|}
\hline \multirow{2}{*}{$\begin{array}{c}\text { Pipe } \\
\text { Size } \\
\text { (inch) } \\
\end{array}$} & \multicolumn{8}{|c|}{ Process Fluid Temperature (F) } \\
\hline & 150 & $\underline{200}$ & $\underline{250}$ & $\underline{300}$ & $\underline{350}$ & $\underline{400}$ & 450 & $\underline{500}$ \\
\hline 1 & 1.0 & 1.5 & 1.5 & 1.5 & 1.5 & 1.5 & 1.5 & 1.5 \\
\hline 2 & 1.5 & 1.5 & 1.5 & 2.0 & 2.0 & 2.5 & 2.5 & 2.5 \\
\hline 3 & 1.5 & 1.5 & 2.0 & 2.0 & 2.0 & 2.5 & 3.0 & 3.0 \\
\hline 4 & 1.5 & 1.5 & 2.0 & 2.5 & 2.5 & 2.5 & 3.0 & 3.0 \\
\hline 5 & 1.5 & 1.5 & 2.0 & 2.5 & 2.5 & 2.5 & 3.0 & 3.0 \\
\hline 6 & 1.5 & 1.5 & 2.0 & 2.5 & 2.5 & 2.5 & 3.0 & 3.0 \\
\hline 8 & 1.5 & 2.0 & 2.0 & 2.5 & 2.5 & 3.0 & 3.5 & 3.5 \\
\hline 10 & 1.5 & 2.0 & 2.5 & 2.5 & 3.0 & 3.0 & 3.5 & 3.5 \\
\hline 12 & 1.5 & 2.0 & 2.5 & 2.5 & 3.0 & 3.0 & 3.5 & 3.5 \\
\hline 14 & 1.5 & 2.0 & 2.5 & 2.5 & 3.0 & 3.0 & 3.5 & 3.5 \\
\hline 16 & 1.5 & 2.0 & 2.5 & 3.0 & 3.0 & 3.5 & 3.5 & 4.0 \\
\hline 18 & 1.5 & 2.0 & 2.5 & 3.0 & 3.0 & 3.5 & 3.5 & 4.0 \\
\hline
\end{tabular}

Note: Energy Cost $=\$ 3.00 / \mathrm{MB} t u$

Return Pipe Temperature $=150 \mathrm{~F}$ 
TABLE A-2.a

Maximum Allowable Heat Losses (Btu/h.ft) from the Supply

Pipe (S) and the Return Pipe (R) for Energy Cost of $\$ 4 / M$ Btu

\begin{tabular}{|c|c|c|c|c|c|c|c|c|c|}
\hline \multirow{3}{*}{$\begin{array}{l}\text { Pipe } \\
\text { Size } \\
\text { (inch) } \\
\end{array}$} & \multirow[b]{2}{*}{ Pipe } & \multicolumn{8}{|c|}{ Process Fluid Temperature (F) } \\
\hline & & & & & & & & & \\
\hline & Type & $\underline{150}$ & $\underline{200}$ & $\underline{250}$ & $\underline{300}$ & $\underline{350}$ & 400 & 450 & $\underline{500}$ \\
\hline \multirow[t]{2}{*}{1} & $S$ & 17.6 & 26.2 & 35.3 & 45.1 & 55.6 & 57.0 & 66.7 & 76.6 \\
\hline & $\mathrm{R}$ & 17.6 & 17.3 & 17.1 & 16.8 & 16.5 & 14.1 & 13.9 & 13.7 \\
\hline \multirow[t]{2}{*}{2} & $S$ & 25.0 & 37.3 & 42.2 & 54.0 & 66.6 & 70.0 & 81.8 & 94.0 \\
\hline & $\mathrm{R}$ & 25.0 & 24.6 & 20.4 & 20.0 & 19.6 & 17.2 & 16.9 & 16.6 \\
\hline \multirow[t]{2}{*}{3} & $S$ & 32.3 & 40.0 & 54.1 & 69.2 & 85.3 & 88.6 & 92.6 & 106.5 \\
\hline & $R$ & 32.3 & 26.3 & 25.8 & 25.3 & 24.7 & 21.4 & 19.0 & 18.7 \\
\hline \multirow[t]{2}{*}{4} & $S$ & 38.5 & 47.4 & 55.3 & 70.7 & 87.3 & 104.6 & 108.6 & 124.9 \\
\hline & $\mathrm{R}$ & 38.5 & 31.1 & 26.5 & 26.0 & 25.4 & 24.8 & 22.0 & 21.5 \\
\hline \multirow[t]{2}{*}{5} & $S$ & 44.8 & 55.1 & 64.0 & 81.8 & 101.0 & 121.0 & 125.0 & 144.0 \\
\hline & $\mathrm{R}$ & 44.8 & 35.9 & 30.4 & 29.8 & 29.0 & 28.3 & 25.0 & 24.4 \\
\hline \multirow[t]{2}{*}{6} & S & 51.0 & 62.6 & 72.4 & 92.7 & 114.4 & 137.2 & 141.1 & 146.4 \\
\hline & $\mathrm{R}$ & 51.0 & 40.7 & 34.3 & 33.4 & 32.5 & .31 .6 & 27.8 & 25.0 \\
\hline \multirow[t]{2}{*}{8} & $S$ & 50.7 & 76.4 & 88.0 & 112.8 & 122.0 & 146.2 & 153.1 & 176.4 \\
\hline & $\mathrm{R}$ & 50.7 & 49.2 & 41.2 & 40.0 & 34.8 & 33.8 & 30.2 & 29.4 \\
\hline \multirow[t]{2}{*}{10} & $S$ & 59.9 & 90.6 & 104.2 & 133.6 & 144.0 & 154.1 & 180.1 & 188.5 \\
\hline & R & 59.9 & 58.0 & 48.3 & 46.7 & 40.4 & 35.8 & 34.8 & 31.5 \\
\hline \multirow[t]{2}{*}{12} & $S$ & 68.4 & 103.4 & 119.1 & 133.2 & 146.2 & 175.3 & 185.3 & 213.7 \\
\hline & $\mathrm{R}$ & 68.4 & 66.0 & 54.8 & 47.1 & 41.4 & 40.0 & 35.8 & 34.7 \\
\hline \multirow[t]{2}{*}{14} & $S$ & 73.6 & 111.7 & 128.6 & 143.0 & 156.8 & 188.0 & 198.6 & 229.2 \\
\hline & $R$ & 73.6 & 70.8 & 58.8 & 50.1 & 43.8 & 42.4 & 37.8 & 36.5 \\
\hline \multirow[t]{2}{*}{16} & $S$ & 81.4 & 104.5 & 123.4 & 158.4 & 173.6 & 188.0 & 219.7 & 231.9 \\
\hline & $\mathrm{R}$ & 81.4 & 66.6 & 56.6 & 54.6 & 47.7 & 42.4 & 40.9 & 37.0 \\
\hline \multirow[t]{2}{*}{18} & $S$ & 88.6 & 114.2 & 135.1 & 154.0 & 190.1 & 205.7 & 240.5 & 253.7 \\
\hline & R & 88.6 & 72.4 & 61.4 & 53.4 & 51.4 & 45.6 & 43.9 & 39.6 \\
\hline
\end{tabular}

Note: Energy Cost $=\$ 4.00 / \mathrm{MBtu}$

Return Pipe Temperature $=150 \mathrm{~F}$ 
TABLE $A-2 \cdot b$

Economic Insulation Thickness (inch) for Hot Viater Supply and Return Pipes for Energy Cost of $\$ 4 / \mathrm{MBtu}$

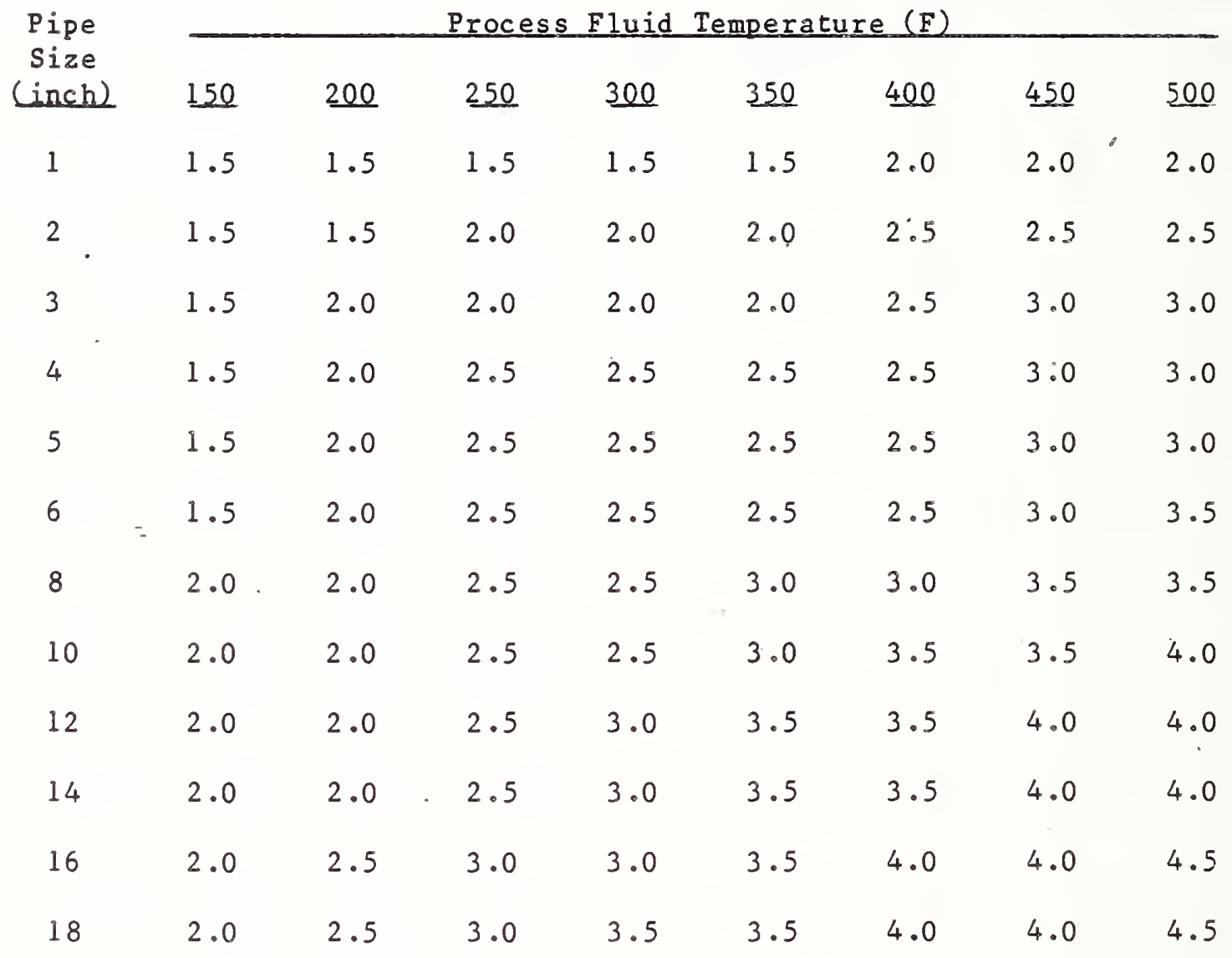

Note: Energy Cost $=\$ 4.00 / \mathrm{MBti}$

Return Pipe Temperature $=150 \mathrm{~F}$ 
TABLE A-3.a

Maximum Allowable Heat Losses (Btu/h.ft) from the Supply

Pipe (S) and the Return Pipe (R) for Energy Cost of $\$ 5 / M$ Btu

\begin{tabular}{|c|c|c|c|c|c|c|c|c|c|}
\hline \multirow{3}{*}{$\begin{array}{l}\text { Pipe } \\
\text { Size } \\
\text { (inch) } \\
\end{array}$} & \multirow[b]{2}{*}{ Pipe } & \multicolumn{8}{|c|}{ Process Fluid Temperature (F) } \\
\hline & & & & & & & & & \\
\hline & Type & $\underline{150}$ & $\underline{200}$ & $\underline{250}$ & $\underline{300}$ & $\underline{350}$ & $\underline{400}$ & $\underline{450}$ & $\underline{500}$ \\
\hline \multirow[t]{2}{*}{1} & $\mathrm{~S}$ & 17.6 & 26.2 & 35.3 & 38.6 & 47.6 & 57.0 & 66.7 & 68.4 \\
\hline & $\mathrm{R}$ & 17.6 & 17.3 & 17.1 & 14.5 & 14.3 & 14.1 & 13.9 & 12.4 \\
\hline \multirow[t]{2}{*}{2} & $S$ & 25.0 & 37.3 & 42.2 & 47.4 & 58.4 & 70.0 & 81.8 & 84.9 \\
\hline & $\mathrm{R}$ & 25.0 & 24.6 & 20.4 & 17.7 & 17.5 & 17.2 & 16.9 & 15.2 \\
\hline \multirow[t]{2}{*}{3} & $S$ & 26.8 & 40.0 & 54.1 & 60.0 & 74.0 & 79.3 & 92.6 & 106.5 \\
\hline & $\mathrm{R}$ & 26.8 & 26.3 & 25.8 & 22.2 & 21.8 & 19.4 & 19.0 & 18.7 \\
\hline \multirow[t]{2}{*}{4} & $\mathrm{~S}$ & 31.7 & 47.4 & 55.3 & 70.7 & 87.3 & 92.9 & 108.6 & 124.9 \\
\hline & $\mathrm{R}$ & 31.7 & 31.1 & 26.5 & 26.0 & 25.4 & 22.5 & 22.0 & 21.5 \\
\hline \multirow[t]{2}{*}{5} & S & 36.8 & 55.1 & 64.0 & 81.8 & 101.0 & 107.0 & 125.0 & 144.0 \\
\hline & $\mathrm{R}$ & 36.8 & 35.9 & 30.4 & 29.8 & 29.0 & 25.6 & 25.0 & 24.4 \\
\hline \multirow{3}{*}{6} & & & & & & & & & \\
\hline & $S$ & 41.7 & 62.6 & 72.4 & 92.7 & 114.4 & 120.8 & 127.1 & 146.4 \\
\hline & $\mathrm{R}$ & 41.7 & 40.7 & 34.3 & 33.4 & 32.5 & 28.5 & 25.6 & 25.0 \\
\hline \multirow[t]{2}{*}{8} & $S$ & 50.7 & 76.4 & 77.2 & 98.8 & 122.0 & 131.0 & 139.5 & 160.7 \\
\hline & $\mathrm{R}$ & 50.7 & 49.2 & 36.6 & 35.7 & 34.8 & 30.9 & 28.0 & 27.4 \\
\hline \multirow[t]{2}{*}{10} & S & 59.9 & 76.8 & 91.1 & 116.7 & 128.6 & 154.1 & 163.6 & 173.1 \\
\hline & $\mathrm{R}$ & 59.9 & 49.7 & 42.8 & 41.7 & 36.8 & 35.8 & 32.3 & 29.4 \\
\hline \multirow[t]{2}{*}{12} & $S$ & 68.4 & 87.8 & 103.9 & 118.5 & 146.2 & 158.6 & 185.3 & 195.8 \\
\hline & $\mathrm{R}$ & 68.4 & 56.6 & 48.5 & 42.5 & 41.3 & 36.8 & 35.8 & 32.4 \\
\hline \multirow[t]{2}{*}{14} & $S$ & 73.6 & 94.7 & 111.5 & 127.1 & 156.8 & 170.0 & 198.6 & 209.8 \\
\hline & $\mathrm{R}$ & 73.6 & 60.8 & 51.7 & 45.2 & 43.8 & 39.0 & 37.8 & 34.2 \\
\hline \multirow[t]{2}{*}{16} & $S$ & 81.4 & 90.9 & 109.7 & 140.6 & 156.8 & 172.0 & 201.0 & 231.9 \\
\hline & $\mathrm{R}$ & 81.4 & 58.5 & 50.9 & 49.3 & 43.8 & 39.4 & 38.2 & 37.0 \\
\hline \multirow[t]{2}{*}{18} & $S$ & 88.6. & 99.4 & 120.0 & 154.0 & 171.5 & 188.1 & 219.8 & 234.2 \\
\hline & $\mathrm{R}$ & 88.6 & 63.6 & 55.3 & 53.4 & 47.3 & 42.5 & 41.1 & 37.4 \\
\hline
\end{tabular}

Note: Energy Cost $=\$ 5.00 / \mathrm{MBtu}$

Return Pipe Temperature $=150 \mathrm{~F}$ 
TABLE $A-3 \cdot b$

Economic Insulation Thickness (inch) for Hot Water Supply and Return Pipes for Energy Cost of $\$ 5 / M B t u$

\begin{tabular}{ccccccccc}
$\begin{array}{c}\text { Pipe } \\
\text { Size } \\
\text { (inch) }\end{array}$ & 150 & $\underline{7}$ Process Fluid & Temperature (E) \\
\cline { 2 - 8 } 1 & 1.5 & 1.5 & 1.5 & 2.0 & 2.0 & 2.0 & 2.0 & 2.5 \\
2 & 1.5 & 1.5 & 2.0 & 2.5 & 2.5 & 2.5 & 2.5 & 3.0 \\
3 & 2.0 & 2.0 & 2.0 & 2.5 & 2.5 & 3.0 & 3.0 & 3.0 \\
4 & 2.0 & 2.0 & 2.5 & 2.5 & 2.5 & 3.0 & 3.0 & 3.0 \\
5 & 2.0 & 2.0 & 2.5 & 2.5 & 2.5 & 3.0 & 3.0 & 3.0 \\
6 & 2.0 & 2.0 & 2.5 & 2.5 & 2.5 & 3.0 & 3.5 & 3.5 \\
8 & 2.0 & 2.0 & 3.0 & 3.0 & 3.0 & 3.5 & 4.0 & 4.0 \\
10 & 2.0 & 2.5 & 3.0 & 3.0 & 3.5 & 3.5 & 4.0 & 4.5 \\
12 & 2.0 & 2.5 & 3.0 & 3.5 & 3.5 & 4.0 & 4.0 & 4.5 \\
14 & 2.0 & 2.5 & 3.0 & 3.5 & 3.5 & 4.0 & 4.0 & 4.5 \\
16 & 2.0 & 3.0 & 3.5 & 3.5 & 4.0 & 4.5 & 4.5 & 4.5 \\
18 & 2.0 & 3.0 & 3.5 & 3.5 & 4.0 & 4.5 & 4.5 & 5.0
\end{tabular}

Note: Energy Cost $=\$ 5.00 / \mathrm{MBtu}$

Return Pipe Temperature $=150 \mathrm{~F}$ 
TABLE A-4.a

Maximum Allowable Heat Losses (Btu/h.ft) from the Supply Pipe (S) and the Return Pipe (R) for Energy Cost of $\$ 6 / M$ Btu

\begin{tabular}{|c|c|c|c|c|c|c|c|c|c|}
\hline \multirow{3}{*}{$\begin{array}{l}\text { Pipe } \\
\text { Size } \\
\text { (inch) } \\
\end{array}$} & \multirow[b]{2}{*}{ Pipe } & \multicolumn{8}{|c|}{ Process Fluid Temperature (F) } \\
\hline & & & & & & & & & \\
\hline & Type & $\underline{150}$ & $\underline{200}$ & $\underline{250}$ & $\underline{300}$ & $\underline{350}$ & $\underline{400}$ & $\underline{450}$ & $\underline{500}$ \\
\hline \multirow[t]{2}{*}{1} & $S$ & 17.6 & 26.2 & 35.3 & 38.6 & 47.6 & 51.0 & 59.6 & 68.4 \\
\hline & $\mathrm{R}$ & 17.6 & 17.3 & 17.1 & 14.5 & 14.3 & 12.7 & 12.6 & 12.4 \\
\hline \multirow[t]{2}{*}{2} & $S$ & 25.0 & 31.3 & 37.1 & 47.4 & 58.4 & 63.2 & 73.9 & 84.9 \\
\hline & $\mathrm{R}$ & 25.0 & 20.7 & 18.0 & 17.7 & 17.5 & 15.7 & 15.5 & 15.2 \\
\hline \multirow[t]{2}{*}{3} & S & 26.8 & 40.0 & 46.9 & 60.0 & 66.2 & 79.3 & 92.6 & 106.5 \\
\hline & $\mathrm{R}$ & 26.8 & 26.3 & 22.6 & 22.2 & 19.7 & 19.4 & 19.0 & 18.7 \\
\hline \multirow[t]{2}{*}{4} & S & 31.7 & 40.9 & 55.3 & 70.7 & 77.5 & 92.9 & 108.6 & 113.4 \\
\hline & $\mathrm{R}$ & 31.7 & 27.0 & 26.5 & 26.0 & 22.9 & 22.5 & 22.0 & 19.9 \\
\hline \multirow[t]{2}{*}{5} & $S$ & 36.8 & 47.3 & 64.0 & 81.8 & 89.3 & 107.0 & 125.0 & 130.0 \\
\hline & $\mathrm{R}$ & 36.8 & 31.1 & 30.4 & 29.8 & 26.1 & 25.6 & 25.0 & 22.5 \\
\hline \multirow[t]{2}{*}{6} & $S$ & 41.7 & 53.5 & 72.4 & 81.7 & 100.8 & 120.8 & 127.1 & 146.4 \\
\hline & $\mathrm{R}$ & 41.7 & 35.1 & 34.3 & 29.9 & 29.2 & 28.5 & 25.6 & 25.0 \\
\hline \multirow[t]{2}{*}{8} & $S$ & 50.7 & 65.0 & 77.2 & 88.6 & 109.3 & 131.0 & 139.5 & 160.7 \\
\hline & $\mathrm{R}$ & 50.7 & 42.3 & 36.6 & 32.4 & 31.7 & 30.9 & 28.0 & 27.4 \\
\hline \multirow[t]{2}{*}{10} & $S$ & 51.1 & 76.8 & 91.1 & $10 \dot{4} .2$ & 128.6 & 140.0 & 150.3 & 173.1 \\
\hline & $\mathrm{R}$ & 51.1 & 49.7 & 42.8 & 37.8 & 36.8 & 33.1 & 30.1 & 29.4 \\
\hline \multirow[t]{2}{*}{12} & $S$ & 58.2 & 76.7 & 92.5 & 118.5 & 132.3 & 158.6 & 169.9 & 181.3 \\
\hline & $R$ & 58.2 & 49.8 & 43.6 & 42.5 & 37.8 & 36.8 & 33.3 & 30.5 \\
\hline \multirow[t]{2}{*}{14} & $S$ & 62.7 & 82.2 & 99.2 & 127.1 & 141.8 & 170.0 & 181.9 & 194.1 \\
\hline & $\mathrm{R}$ & 62.7 & 53.2 & 46.4 & 45.2 & 40.2 & 39.0 & 35.3 & 32.2 \\
\hline \multirow[t]{2}{*}{16} & $S$ & 69.0 & 90.9 & 109.7 & 127.0 & 143.5 & 172.0 & 201.0 & 214.3 \\
\hline & $\mathrm{R}$ & 69.0 & 58.5 & 50.9 & 45.1 & 40.6 & 39.4 & 38.2 & 34.8 \\
\hline \multirow[t]{2}{*}{18} & $S$ & 75.2 & 99.4 & 120.0 & 139.0 & 156.8 & 173.7 & 203.0 & 218.0 \\
\hline & $\mathrm{R}$ & 75.2 & 63.6 & 55.3 & 48.9 & 43.9 & 39.9 & 38.7 & .35 .4 \\
\hline
\end{tabular}


TABLE $A-4 \cdot b$

$$
\begin{gathered}
\text { Economic Insulation Thickness (inch) for Hot Water Suppiy } \\
\text { and Return Pipes for Energy Cost of } \$ 6 / \mathrm{MBtu}
\end{gathered}
$$

\begin{tabular}{|c|c|c|c|c|c|c|c|c|}
\hline \multirow{2}{*}{$\begin{array}{l}\text { Pipe } \\
\text { Size } \\
\text { (inch) } \\
\end{array}$} & \multicolumn{8}{|c|}{ Process Fluid Temperature (F) } \\
\hline & $\underline{150}$ & $\underline{200}$ & $\underline{250}$ & $\underline{300}$ & $\underline{350}$ & 400 & $\underline{450}$ & $\underline{500}$ \\
\hline 1 & 1.5 & 1.5 & 1.5 & 2.0 & 2.0 & 2.5 & 2.5 & 2.5 \\
\hline 2 & 1.5 & 2.0 & 2.5 & 2.5 & 2.5 & 3.0 & 3.0 & 3.0 \\
\hline 3 & 2.0 & 2.0 & 2.5 & 2.5 & 3.0 & 3.0 & 3.0 & 3.0 \\
\hline 4 & 2.0 & 2.5 & 2.5 & 2.5 & 3.0 & 3.0 & 3.0 & 3.5 \\
\hline 5 & 2.0 & 2.5 & 2.5 & 2.5 & 3.0 & 3.0 & 3.0 & 3.5 \\
\hline 6 & 2.0 & 2.5 & 2.5 & 3.0 & 3.0 & 3.0 & 3.5 & 3.5 \\
\hline 8 & 2.0 & 2.5 & 3.0 & 3.5 & 3.5 & 3.5 & 4.0 & 4.0 \\
\hline 10 & -2.5 & 2.5 & 3.0 & 3.5 & 3.5 & 4.0 & 4.5 & 4.5 \\
\hline 12 & 2.5 & 3.0 & 3.5 & 3.5 & 4.0 & 4.0 & 4.5 & 5.0 \\
\hline 14 & 2.5 & 3.0 & 3.5 & 3.5 & 4.0 & 4.0 & 4.5 & 5.0 \\
\hline 16 & 2.5 & 3.0 & 3.5 & 4.0 & 4.5 & 4.5 & 4.5 & 5.0 \\
\hline 18 & 2.5 & 3.0 & 3.5 & 4.0 & 4.5 & 5.0 & 5.0 & 5.5 \\
\hline
\end{tabular}

Note: Energy Cost $=\$ 6.00 / \mathrm{MBtu}$

Return Pipe Temperature $=150 \mathrm{~F}$ 
TABLE A-5.a

Maximum Allowable Heat Losses (Btu/h.ft) from the Supply

Pipe (S) and the Return Pipe (R) for Energy Cost of $\$ 7 / M$ Btu

\begin{tabular}{|c|c|c|c|c|c|c|c|c|c|}
\hline \multirow{3}{*}{$\begin{array}{c}\text { Pipe } \\
\text { Size } \\
\text { (inch) }\end{array}$} & \multirow[b]{2}{*}{ Pipe } & \multicolumn{8}{|c|}{ Process Fluid Temperature (F) } \\
\hline & & & & & & & & & \\
\hline & Type & $\underline{150}$ & $\underline{200}$ & $\underline{250}$ & $\underline{300}$ & $\underline{350}$ & $\underline{400}$ & $\underline{450}$ & $\underline{500}$ \\
\hline \multirow[t]{2}{*}{1} & $S$ & 17.6 & 26.2 & 30.2 & 38.6 & 47.6 & 51.0 & 59.6 & 62.7 \\
\hline & $\mathrm{R}$ & 17.6 & 17.3 & 14.7 & 14.5 & 14.3 & 12.7 & 12.6 & 11.5 \\
\hline \multirow[t]{2}{*}{2} & $\mathrm{~s}$ & 21.0 & 31.3 & 37.1 & 47.4 & 58.4 & 63.2 & 73.9 & 84.9 \\
\hline & $\mathbf{R}$ & 21.0 & 20.7 & 18.0 & 17.7 & 17.5 & 15.7 & 15.5 & 15.2 \\
\hline \multirow[t]{2}{*}{3} & $S$ & 26.8 & 40.0 & 46.9 & 53.6 & $66 . \dot{2}$ & 79.3 & 92.6 & 106.5 \\
\hline & $\mathbf{R}$ & 26.8 & 26.3 & 22.6 & 20.1 & 19.7 & 19.4 & 19.0 & 18.7 \\
\hline \multirow[t]{2}{*}{4} & $S$ & 27.4 & 40.9 & 55.3 & 62.9 & 77.5 & 92.9 & 98.6 & 113.4 \\
\hline & $\mathrm{R}$ & 27.4 & 27.0 & 26.5 & 23.3 & 22.9 & 22.5 & 20.3 & 19.9 \\
\hline \multirow[t]{2}{*}{5} & $S$ & 31.7 & 47.3 & 64.0 & 72.4 & 89.3 & 107.0 & 113.0 & 130.0 \\
\hline & R & 31.7 & 31.1 & 30.4 & 26.7 & 26.1 & 25.6 & 23.0 & 22.5 \\
\hline \multirow[t]{2}{*}{6} & $S$ & 35.8 & 53.5 & 72.4 & 81.7 & 100.8 & 108.8 & 116.4 & 133.9 \\
\hline & $\mathrm{R}$ & 35.8 & 35.1 & 34.3 & 29.9 & .29 .2 & 26.1 & 23.8 & 23.3 \\
\hline \multirow[t]{2}{*}{8} & $\mathrm{~S}$ & 43.3 & 65.0 & 77.2 & 88.6 & 109.3 & 119.4 & 128.8 & 148.2 \\
\hline & $\mathrm{R}$ & 43.3 & 42.3 & 36.6 & 32.4 & 31.7 & 28.6 & 26.2 & 25.7 \\
\hline \multirow[t]{2}{*}{10} & $\mathrm{~S}$ & 44.9 & 67.2 & 81.4 & 104.2 & 116.8 & 128.6 & 150.3 & 160.6 \\
\hline & $\mathrm{R}$ & 44.9 & 43.9 & 38.6 & 37.8 & 33.9 & 30.8 & 30.1 & 27.6 \\
\hline \multirow[t]{2}{*}{12} & $S$ & 51.1 & 68.3 & 83.7 & 107.2 & 132.3 & 145.4 & 157.4 & 169.3 \\
\hline & $R$ & 51.1 & 44.6 & 39.7 & 38.8 & 37.8 & 34.2 & 31.3 & 28.8 \\
\hline \multirow[t]{2}{*}{14} & $S$ & 54.7 & 73.2 & 89.7 & 114.9 & 141.8 & 155.7 & 168.4 & 181.1 \\
\hline & $\mathrm{R}$ & 54.7 & 47.7 & 42.3 & 41.3 & 40.2 & 36.2 & 33.1 & 30.5 \\
\hline \multirow[t]{2}{*}{16} & $S$ & 60.3 & 80.9 & 99.1 & 116.3 & 143.5 & 172.0 & 185.8 & 199.7 \\
\hline & $\mathrm{R}$ & 60.3 & 52.4 & 46.4 & 41.7 & 40.6 & 39.4 & 35.9 & 33.0 \\
\hline \multirow[t]{2}{*}{18} & $S$ & 65.8 & 88.4 & 108.4 & 127.1 & 144.9 & 173.7 & 189.0 & 218.0 \\
\hline & $\mathrm{R}$ & 65.8 & 57.0 & 50.4 & 45.2 & 41.0 & 39.9 & 36.6 & 35.4 \\
\hline
\end{tabular}

Note: Energy Cost $=\$ 7.00 / \mathrm{MBtu}$

Return Pipe Temperature $=150 \mathrm{~F}$ 
TABLE $A-5 . b$

Economic Insulation Thickness (inch) for Hot Water Supply and Return Pipes for Energy Cost of $\$ 7 / \mathrm{MBtu}$

$\begin{array}{lllllllll}\begin{array}{c}\text { Pipe } \\ \begin{array}{c}\text { Size } \\ \text { (inch) }\end{array}\end{array} & \begin{array}{l}150 \\$\cline { 3 - 7 }\end{array} & $\underline{200} & \underline{250} & \underline{300} & \underline{350} & \underline{400} & \underline{450} & \underline{500} \\ 1 & 1.5 & 1.5 & 2.0 & 2.0 & 2.0 & 2.5 & 2.5 & 3.0 \\ 2 & 2.0 & 2.0 & 2.5 & 2.5 & 2.5 & 3.0 & 3.0 & 3.0 \\ 3 & 2.0 & 2.0 & 2.5 & 3.0 & 3.0 & 3.0 & 3.0 & 3.0 \\ 4 & 2.5 & 2.5 & 2.5 & 3.0 & 3.0 & 3.0 & 3.5 & 3.5 \\ 5 & 2.5 & 2.5 & 2.5 & 3.0 & 3.0 & 3.0 & 3.5 & 3.5 \\ 6 & 2.5 & 2.5 & 2.5 & 3.0 & 3.0 & 3.5 & 4.0 & 4.0 \\ 8 & 2.5 & 2.5 & 3.0 & 3.5 & 3.5 & 4.0 & 4.5 & 4.5 \\ 10 & -3.0 & 3.0 & 3.5 & 3.5 & 4.0 & 4.5 & 4.5 & 5.0 \\ 12 & 3.0 & 3.5 & 4.0 & 4.0 & 4.0 & 4.5 & 5.0 & 5.5 \\ 14 & 3.0 & 3.5 & 4.0 & 4.0 & 4.0 & 4.5 & 5.0 & 5.5 \\ 16 & 3.0 & 3.5 & 4.0 & 4.5 & 4.5 & 4.5 & 5.0 & 5.5 \\ 18 & 3.0 & 3.5 & 4.0 & 4.5 & 5.0 & 5.0 & 5.5 & 5.5\end{array}$

Note: Energy Cost $=\$ 7.00 / \mathrm{MBtu}$

Return Pipe Temperature $=150 \mathrm{~F}$ 
TABLE A-6.a

Maximum Allowable Heat Losses (Btu/h.ft) from the Supply

Pipe ( $S$ ) and the Return Pipe (R) for Energy Cost of $\$ 8 / M$ Btu

\begin{tabular}{|c|c|c|c|c|c|c|c|c|c|}
\hline \multirow{3}{*}{$\begin{array}{l}\text { Pipe } \\
\text { Size } \\
\text { (inch) }\end{array}$} & \multirow{2}{*}{ Pipe } & \multicolumn{8}{|c|}{ Process Fluid Temperature (F) } \\
\hline & & 150 & 200 & 250 & 300 & 350 & 400 & 450 & 500 \\
\hline & & 130 & $\underline{200}$ & $\underline{250}$ & 300 & 200 & 400 & 400 & $\underline{300}$ \\
\hline \multirow[t]{2}{*}{1} & S & 17.6 & 22.4 & 30.2 & 34.5 & 42.5 & 51.0 & 59.6 & 62.7 \\
\hline & R & 17.6 & 14.9 & 14.7 & 13.1 & 12.9 & 12.7 & 12.6 & 11.5 \\
\hline \multirow[t]{2}{*}{2} & $S$ & 18.5 & 27.5 & 37.1 & 42.8 & 52.8 & 63.2 & 73.9 & 84.9 \\
\hline & $\mathbf{R}$ & 18.5 & 18.3 & 18.0 & 16.1 & 15.9 & 15.7 & 15.5 & 15.2 \\
\hline \multirow[t]{2}{*}{3} & $S$ & 23.3 & 34.8 & 42.0 & 53.6 & 66.2 & 79.3 & 92.6 & 106.5 \\
\hline & $\mathbf{R}$ & 23.3 & 23.0 & 20.4 & 20.1 & 19.7 & 19.4 & 19.0 & 18.7 \\
\hline \multirow[t]{2}{*}{4} & $\mathrm{~S}$ & 27.4 & 40.9 & 49.2 & 62.9 & 70.4 & 84.4 & 98.6 & 113.4 \\
\hline & $\mathrm{R}$ & 27.4 & 27.0 & 23.7 & 23.3 & 21.0 & 20.7 & 20.3 & 19.9 \\
\hline \multirow[t]{2}{*}{5} & $S$ & 31.7 & 47.3 & 56.6 & 72.4 & 80.7 & 96.7 & 113.0 & 119.4 \\
\hline & R & 31.7 & 31.1 & 27.2 & 26.7 & 23.9 & 23.4 & 23.0 & 21.0 \\
\hline \multirow[t]{2}{*}{6} & S & 35.8 & 53.5 & 63.9 & 73.6 & 90.8 & 108.8 & 116.4 & 133.9 \\
\hline & $\mathbf{R}$ & 35.8 & 35.1 & 30.5 & 27.2 & 26.7 & $26: 1$ & 23.8 & 23.3 \\
\hline \multirow[t]{2}{*}{8} & $\mathrm{~S}$ & 43.3 & 57.1 & 69.3 & 80.8 & 99.6 & 119.4 & 128.8 & 148.2 \\
\hline & R & 43.3 & 37.4 & 33.1 & 29.8 & 29.2 & 28.6 & 26.2 & 25.7 \\
\hline \multirow[t]{2}{*}{10} & $S$ & 44.9 & 67.2 & 81.4 & 94.7 & 107.3 & 128.6 & 150.3 & 160.6 \\
\hline & $\mathrm{R}$ & 44.9 & 43.9 & 38.6 & 34.7 & 31.4 & 30.8 & 30.1 & 27.6 \\
\hline \multirow[t]{2}{*}{12} & $S$ & 51.1 & 68.3 & 83.7 & 107.2 & 121.3 & 134.7 & 157.4 & 169.3 \\
\hline & R & 51.1 & 44.6 & 39.7 & 38.8 & 35.0 & 32.0 & 31.3 & 28.8 \\
\hline \multirow[t]{2}{*}{14} & $S$ & 54.7 & 73.2 & 89.7 & 114.9 & 129.9 & 144.1 & 157.2 & 181.1 \\
\hline & $\mathbf{R}$ & 54.7 & 47.7 & 42.3 & 41.3 & 37.2 & 33.9 & 31.3 & 30.5 \\
\hline \multirow[t]{2}{*}{16} & $S$ & 53.8 & 73.1 & 90.8 & 116.3 & 132.6 & 159.0 & 173.2 & 199.7 \\
\hline & $\mathrm{R}$ & 53.8 & 47.6 & 42.8 & 41.7 & 38.0 & 37.0 & 34.0 & 33.0 \\
\hline \multirow[t]{2}{*}{18} & $S$ & 58.7 & 79.9 & 99.2 & 127.1 & 134.9 & 161.8 & 189.0 & 218.0 \\
\hline & $\mathbf{R}$ & 58.7 & 51.8 & 46.5 & 45.2 & 38.6 & 37.6 & 36.6 & 35.4 \\
\hline
\end{tabular}

Note: Energy Cost $=\$ 8.00 / \mathrm{MBtu}$

Return Pipe Temperature $=150 \mathrm{~F}$ 
TABLE $A-6 \cdot b$ Economic Insulation Thickness (inch) for Hot Water Supply
and Return Pipes for Energy Cost of $\$ 8 /$ MBtu

\begin{tabular}{ccccccccc}
$\begin{array}{c}\text { Pipe } \\
\text { Size } \\
\text { (inch) }\end{array}$ & 150 & $\underline{700}$ & $\underline{250}$ & $\underline{300}$ & $\underline{350}$ & $\underline{400}$ & $\underline{450}$ & $\underline{500}$ \\
\cline { 2 - 8 } 1 & 1.5 & 2.0 & 2.0 & 2.5 & 2.5 & 2.5 & 2.5 & 3.0 \\
2 & 2.5 & 2.5 & 2.5 & 3.0 & 3.0 & 3.0 & 3.0 & 3.0 \\
3 & 2.5 & 2.5 & 3.0 & 3.0 & 3.0 & 3.0 & 3.0 & 3.0 \\
4 & 2.5 & 2.5 & 3.0 & 3.0 & 3.5 & 3.5 & 3.5 & 3.5 \\
5 & 2.5 & 2.5 & 3.0 & 3.0 & 3.5 & 3.5 & 3.5 & 4.0 \\
6 & 2.5 & 2.5 & 3.0 & 3.5 & 3.5 & 3.5 & 4.0 & 4.0 \\
8 & 2.5 & 3.0 & 3.5 & 4.0 & 4.0 & 4.0 & 4.5 & 4.5 \\
10 & 3.0 & 3.0 & 3.5 & 4.0 & 4.5 & 4.5 & 4.5 & 5.0 \\
12 & 3.0 & 3.5 & 4.0 & 4.0 & 4.5 & 5.0 & 5.0 & 5.5 \\
14 & 3.0 & 3.5 & 4.0 & 4.0 & 4.5 & 5.0 & 5.5 & 5.5 \\
16 & 3.5 & 4.0 & 4.5 & 4.5 & 5.0 & 5.0 & 5.5 & 5.5 \\
18 & 3.5 & 4.0 & 4.5 & 4.5 & 5.5 & 5.5 & 5.5 & 5.5
\end{tabular}

Note: Energy Cost $=\$ 8.00 / \mathrm{MBtu}$

Return Pipe Temperature $=150 \mathrm{~F}$ 
TABLE A-7.a

Maximum Allowable Heat Losses (Btu/h.ft) from the Supply

Pipe (S) and the Return Pipe (R) for Energy Cost of $\$ 9 / M$ Btu

\begin{tabular}{|c|c|c|c|c|c|c|c|c|c|}
\hline \multirow{2}{*}{$\begin{array}{l}\text { Pipe } \\
\text { Size } \\
\text { (inch) } \\
\end{array}$} & \multirow[b]{2}{*}{ Pipe } & \multicolumn{8}{|c|}{ Process Fluid Temperature (F) } \\
\hline & & 150 & $\underline{200}$ & $\underline{250}$ & $\underline{300}$ & $\underline{350}$ & 400 & 450 & $\underline{500}$ \\
\hline \multirow[t]{2}{*}{1} & $S$ & 17.6 & 22.4 & 30.2 & 34.5 & 42.5 & 46.7 & 54.6 & 62.7 \\
\hline & $\mathrm{R}$ & 17.6 & 14.9 & 14.7 & 13.1 & 12.9 & 11.8 & 11.6 & 11.5 \\
\hline \multirow{2}{*}{2} & $S$ & 18.5 & 27.5 & 37.1 & 42.8 & 52.8 & 63.2 & 73.9 & 84.9 \\
\hline & $\mathrm{R}$ & 18.5 & 18.3 & 18.0 & 16.1 & 15.9 & 15.7 & 15.5 & 15.2 \\
\hline \multirow[t]{2}{*}{3} & $S$ & 23.3 & 34.8 & 42.0 & 53.6 & 66.2 & 79.3 & 84.6 & 97.2 \\
\hline & $\mathrm{R}$ & 23.3 & 23.0 & 20.4 & 20.1 & 19.7 & 19.4 & 17.6 & 17.3 \\
\hline \multirow[t]{2}{*}{4} & $S$ & 27.4 & 40.9 & 49.2 & 62.9 & 70.4 & 84.4 & 98.6 & 113.4 \\
\hline & $\mathbf{R}$ & 27.4 & 27.0 & 23.7 & 23.3 & 21.0 & 20.7 & 20.3 & 19.9 \\
\hline \multirow[t]{2}{*}{5} & $S$ & 31.7 & 47.3 & 56.6 & 72.4 & 30.7 & 96.7 & 103.8 & 119.4 \\
\hline & $\mathrm{R}$ & 31.7 & 31.1 & 27.2 & 26.7 & 23.9 & 23.4 & 21.4 & 21.0 \\
\hline \multirow[t]{2}{*}{6} & $S$ & 35.8 & 53.5 & 63.9 & 73.6 & 90.8 & 108.8 & 116.4 & 124.1 \\
\hline & $\mathrm{R}$ & 35.8 & 35.1 & 30.5 & 27.2 & 26.7 & 26.1 & 23.8 & 21.9 \\
\hline \multirow[t]{2}{*}{8} & $S$ & 43.3 & 57.1 & 69.3 & 80.8 & 99.6 & 110.2 & 128.8 & 138.1 \\
\hline & $\mathrm{R}$ & 43.3 & 37.4 & 33.1 & 29.8 & 29.2 & 26.7 & 26.2 & 24.3 \\
\hline \multirow[t]{2}{*}{10} & $S$ & 44.9 & 60.2 & 74.0 & 94.7 & 107.3 & 128.6 & 139.5 & 150.3 \\
\hline & $\mathrm{R}$ & 44.9 & 39.5 & 35.4 & 34.7 & 31.4 & 30.8 & 28.2 & 26.1 \\
\hline \multirow[t]{2}{*}{12} & S & 51.1 & 68.3 & 83.7 & 98.3 & 112.4 & 134.7 & 147.1 & 169.3 \\
\hline & $\mathrm{R}$ & 51.1 & 44.6 & 39.7 & 35.9 & 32.7 & 32.0 & 29.5 & 28.8 \\
\hline \multirow[t]{2}{*}{14} & $S$ & 54.7 & 73.2 & 89.7 & 105.3 & 120.2 & 134.5 & 157.2 & 170.2 \\
\hline & $\mathrm{R}$ & 54.7 & 47.7 & 42.3 & 38.2 & 34.8 & 32.0 & 31.3 & 29.0 \\
\hline \multirow[t]{2}{*}{16} & $S$ & 53.8 & 73.1 & 90.8 & 116.3 & 132.6 & 148.2 & 173.2 & 187.4 \\
\hline & $\mathrm{R}$ & 53.8 & 47.6 & 42.8 & 41.7 & 38.0 & 34.8 & 34.0 & 31.4 \\
\hline \multirow[t]{2}{*}{18} & $\begin{array}{l}S \\
R\end{array}$ & $\begin{array}{l}58.7 \\
58.7\end{array}$ & $\begin{array}{l}79.9 \\
51.8\end{array}$ & $\begin{array}{l}99.2 \\
46.5\end{array}$ & $\begin{array}{r}117.4 \\
42.2\end{array}$ & $\begin{array}{r}134.9 \\
38.6\end{array}$ & $\begin{array}{r}161.8 \\
37.6\end{array}$ & $\begin{array}{r}189.0 \\
. \quad 36.6\end{array}$ & $\begin{array}{r}218.0 \\
35.4\end{array}$ \\
\hline & & & & & & 38.0 & & 36.6 & 35.4 \\
\hline
\end{tabular}

Note: Energy Cost $=\$ 9.00 / \mathrm{MBtu}$

Return Pipe Temperature $=150 \mathrm{~F}$ 
TABLE $A-7 . b$

Economic Insulation Thickness (inch) for Hot Water Supply and Return Pipes for Energy Cost of $\$ 9 / M B t u$

\begin{tabular}{|c|c|c|c|c|c|c|c|c|}
\hline \multirow{3}{*}{$\begin{array}{l}\text { Pipe } \\
\text { Size } \\
\text { (inch) }\end{array}$} & \multicolumn{8}{|c|}{ Process Fluid Temperature (F) } \\
\hline & & & & & & & & \\
\hline & $\underline{150}$ & $\underline{200}$ & $\underline{250}$ & $\underline{300}$ & $\underline{350}$ & 400 & 450 & $\underline{500}$ \\
\hline 1 & 1.5 & 2.0 & 2.0 & 2.5 & 2.5 & 3.0 & 3.0 & 3.0 \\
\hline 2 & 2.5 & 2.5 & 2.5 & 3.0 & 3.0 & 3.0 & 3.0 & 3.0 \\
\hline 3 & 2.5 & 2.5 & 3.0 & 3.0 & 3.0 & 3.0 & 3.5 & 3.5 \\
\hline 4 & 2.5 & 2.5 & 3.0 & 3.0 & 3.5 & 3.5 & 3.5 & 3.5 \\
\hline 5 & 2.5 & 2.5 & 3.0 & 3.0 & 3.5 & 3.5 & 4.0 & 4.0 \\
\hline 6 & 2.5 & 2.5 & 3.0 & 3.5 & 3.5 & 3.5 & 4.0 & 4.5 \\
\hline 8 & 2.5 & 3.0 & 3.5 & 4.0 & 4.0 & 4.5 & 4.5 & 5.0 \\
\hline 10 & -3.0 & 3.5 & 4.0 & 4.0 & 4.5 & 4.5 & 5.0 & 5.5 \\
\hline 12 & 3.0 & 3.5 & 4.0 & 4.5 & 5.0 & 5.0 & 5.5 & 5.5 \\
\hline 14 & 3.0 & 3.5 & 4.0 & 4.5 & 5.0 & 5.5 & 5.5 & 6.0 \\
\hline 16 & 3.5 & 4.0 & 4.5 & 4.5 & 5.0 & 5.5 & 5.5 & 6.0 \\
\hline 18 & 3.5 & 4.0 & 4.5 & 5.0 & 5.5 & 5.5 & 5.5 & 6.0 \\
\hline
\end{tabular}

Note: Energy Cost $=\$ 9.00 / \mathrm{MBtu}$

Return Pipe Temperature $=150 \mathrm{~F}$ 
TABLE A-8.a

Maximum Allowable Heat Losses (Btu/h.ft) from the Supply Pipe (S) and the Return Pipe (R) for Energy Cost of $\$ 10 / M$ Btu

\begin{tabular}{|c|c|c|c|c|c|c|c|c|c|}
\hline \multirow{3}{*}{$\begin{array}{l}\text { Pipe } \\
\text { Size } \\
\text { (inch) } \\
\end{array}$} & \multirow[b]{2}{*}{ Pipe } & \multicolumn{8}{|c|}{ Process Fluid Temperature (F) } \\
\hline & & & & & & & & & \\
\hline & Type & $\underline{150}$ & $\underline{200}$ & $\underline{250}$ & $\underline{300}$ & $\underline{350}$ & $\underline{400}$ & $\underline{450}$ & $\underline{500}$ \\
\hline \multirow[t]{2}{*}{1} & $\mathrm{~S}$ & 17.6 & 22.4 & 27.0 & 34.5 & 39.0 & 46.7 & 54.6 & 62.7 \\
\hline & $\mathrm{R}$ & 17.6 & 14.9 & 13.2 & 13.1 & 11.9 & 11.8 & 11.6 & 11.5 \\
\hline \multirow[t]{2}{*}{2} & $S$ & 18.5 & 27.5 & 37.1 & 42.8 & 52.8 & 63.2 & 73.9 & 84.9 \\
\hline & $\mathrm{R}$ & 18.5 & 18.3 & 18.0 & 16.1 & 15.9 & 15.7 & 15.5 & 15.2 \\
\hline \multirow[t]{2}{*}{3} & $S$ & 23.3 & 34.8 & 42.0 & 53.6 & 66.2 & 79.3 & 84.6 & 97.2 \\
\hline & $\mathrm{R}$ & 23.3 & 23.0 & 20.4 & 20.1 & 19.7 & 19.4 & 17.6 & 17.3 \\
\hline \multirow[t]{2}{*}{4} & $S$ & 27.4 & 40.9 & 49.2 & 57.1 & 70.4 & 84.4 & 98.6 & 113.4 \\
\hline & $\mathrm{R}$ & 27.4 & 27.0 & 23.7 & 21.4 & 21.0 & 20.7 & 20.3 & 19.9 \\
\hline \multirow[t]{2}{*}{5} & $S$ & 31.7 & 47.3 & 56.6 & 65.4 & 80.7 & 88.8 & 103.8 & 119.4 \\
\hline & $\mathrm{R}$ & 31.7 & 31.1 & 27.2 & 24.3 & 23.9 & 21.8 & 21.4 & 21.0 \\
\hline \multirow[t]{2}{*}{6} & $S$ & 35.8 & 47.2 & 57.6 & 73.6 & 90.8 & 99.6 & 107.9 & 124.1 \\
\hline & $\mathrm{R}$ & 35.8 & 31.1 & 27.7 & 27.2 & .26 .7 & 24.2 & 22.3 & 21.9 \\
\hline \multirow[t]{2}{*}{8} & $S$ & 38.2 & 51.2 & 63.2 & 74.6 & 92.0 & 110.2 & 120.1 & 138.1 \\
\hline & $\mathrm{R}$ & 38.2 & 33.7 & 30.4 & 27.7 & 27.3 & 26.7 & 24.7 & 24.3 \\
\hline \multirow[t]{2}{*}{10} & $S$ & 44.9 & 60.2 & 74.0 & 87.0 & 99.6 & 119.4 & 130.6 & 150.3 \\
\hline & $\mathrm{R}$ & 44.9 & 39.5 & 35.4 & 32.1 & 29.4 & 28.8 & 26.6 & 26.1 \\
\hline \multirow[t]{2}{*}{12} & $S$ & 45.6 & 61.9 & 76.8 & 98.3 & 112.4 & 125.8 & 147.1 & 159.3 \\
\hline & $\mathrm{R}$ & 45.6 & 40.6 & 36.6 & 35.9 & 32.7 & 30.2 & 29.5 & 27.4 \\
\hline \multirow[t]{2}{*}{14} & $S$ & 48.8 & 66.3 & 82.2 & 105.3 & 120.2 & 134.5 & 157.2 & 170.2 \\
\hline & $\mathrm{R}$ & 48.8 & 43.3 & 39.0 & 38.2 & 34.8 & 32.0 & 31.3 & 29.0 \\
\hline \multirow[t]{2}{*}{16} & $S$ & 53.8 & 73.1 & 90.8 & 116.3 & 132.6 & 148.2 & 162.6 & 187.4 \\
\hline & $\mathrm{R}$ & 53.8 & 47.6 & 42.8 & 41.7 & 38.0 & 34.8 & 32.2 & 31.4 \\
\hline \multirow[t]{2}{*}{18} & $S$ & 58.7 & 73.2 & 91.6 & 117.4 & 134.9 & 161.8 & 177.3 & 204.4 \\
\hline & $\mathrm{R}$ & 58.7 & 47.7 & 43.2 & 42.2 & 38.6 & 37.6 & 34.7 & 33.7 \\
\hline
\end{tabular}

Note: Energy Cost $=\$ 10.00 / \mathrm{MBtu}$

Return Pipe Temperature $=150 \mathrm{~F}$ 
TABLE $A-8 \cdot b$

\begin{abstract}
Economic Insulation Thickness (inch) for Hot Water Supply and Return Pipes for Energy Cost of $\$ 10 / \mathrm{MBtu}$
\end{abstract}

\begin{tabular}{|c|c|c|c|c|c|c|c|c|}
\hline \multirow{2}{*}{$\begin{array}{c}\text { Pipe } \\
\text { Size } \\
\text { (inch) }\end{array}$} & \multicolumn{8}{|c|}{ Process Fluid Temperature (F) } \\
\hline & 150 & $\underline{200}$ & $\underline{250}$ & 300 & 350 & 400 & 450 & $\underline{500}$ \\
\hline 1 & 1.5 & 2.0 & 2.5 & 2.5 & 3.0 & 3.0 & 3.0 & 3.0 \\
\hline 2 & 2.5 & 2.5 & 2.5 & 3.0 & 3.0 & 3.0 & 3.0 & 3.0 \\
\hline 3 & 2.5 & 2.5 & 3.0 & 3.0 & 3.0 & 3.0 & 3.5 & 3.5 \\
\hline 4 & 2.5 & 2.5 & 3.0 & 3.5 & 3.5 & 3.5 & 3.5 & 3.5 \\
\hline 5 & 2.5 & 2.5 & 3.0 & 3.5 & 3.5 & 4.0 & 4.0 & 4.0 \\
\hline 6 & 2.5 & 3.0 & 3.5 & 3.5 & 3.5 & 4.0 & 4.5 & 4.5 \\
\hline 8 & 3.0 & 3.5 & 4.0 & 4.5 & 4.5 & 4.5 & 5.0 & 5.0 \\
\hline 10 & 3.0 & 3.5 & 4.0 & 4.5 & 5.0 & 5.0 & 5.5 & 5.5 \\
\hline $1: 2$ & 3.5 & 4.0 & 4.5 & 4.5 & 5.0 & 5.5 & 5.5 & 6.0 \\
\hline 14 & 3.5 & 4.0 & 4.5 & 4.5 & 5.0 & 5.5 & 5.5 & 6.0 \\
\hline 16 & 3.5 & 4.0 & 4.5 & 4.5 & 5.0 & 5.5 & 6.0 & 6.0 \\
\hline 18 & 3.5 & 4.5 & 5.0 & 5.0 & 5.5 & 5.5 & 6.0 & 6.0 \\
\hline
\end{tabular}

Note: Energy Cost $=\$ 10.00 / \mathrm{MBtu}$

Return Pipe Temperature $=150 \mathrm{~F}$ 
TABLE A-9.a

Maximum Allowable Heat Losses (Btu/h.ft) from the Supply Pipe (S) and the Return Pipe (R) for Energy Cost of $\$ 11 / M$ Btu

\begin{tabular}{|c|c|c|c|c|c|c|c|c|c|}
\hline \multirow{3}{*}{$\begin{array}{l}\text { Pipe } \\
\text { Size } \\
\text { (inch) } \\
\end{array}$} & \multirow[b]{2}{*}{ Pipe } & \multicolumn{8}{|c|}{ Process Fluid Temperature (F) } \\
\hline & & & & & & & & & \\
\hline & Type & $\underline{150}$ & $\underline{200}$ & $\underline{250}$ & $\underline{300}$ & $\underline{3.50}$ & $\underline{400}$ & $\underline{450}$ & $\underline{500}$ \\
\hline \multirow[t]{2}{*}{1} & $S$ & 15.1 & 22.4 & 27.0 & 34.5 & 39.0 & 46.7 & 54.6 & 62.7 \\
\hline & $\mathrm{R}$ & 15.1 & 14.9 & 13.2 & 13.1 & 11.9 & 11.8 & 11.6 . & 11.5 \\
\hline \multirow[t]{2}{*}{2} & $S$ & 18.5 & 27.5 & 33.5 & 42.8 & 52.8 & 63.2 & 73.9 & 78.2 \\
\hline & $\mathrm{R}$ & 18.5 & 18.3 & 16.4 & 16.1 & 15.9 & 15.7 & 15.5 & 14.2 \\
\hline \multirow[t]{2}{*}{3} & $S$ & 23.3 & 34.8 & 42.0 & 53.6 & 66.2 & 79.3 & 84.6 & 97.2 \\
\hline & $\mathrm{R}$ & 23.3 & 23.0 & 20.4 & 20.1 & 19.7 & 19.4 & 17.6 & 17.3 \\
\hline \multirow[t]{2}{*}{4} & $S$ & 27.4 & 40.9 & 44.7 & 57.1 & 70.4 & 84.4 & 98.6 & 113.4 \\
\hline & $\mathrm{R}$ & 27.4 & 27.0 & 21.7 & 21.4 & 21.0 & 20.7 & 20.3 & 19.9 \\
\hline \multirow[t]{2}{*}{5} & $S$ & 31.7 & 41.9 & 51.2 & 65.4 & 74.1 & 88.8 & 103.8 & 119.4 \\
\hline & $\mathrm{R}$ & 31.7 & 27.7 & 24.7 & 24.3 & 22.2 & 21.8 & 21.4 & 21.0 \\
\hline \multirow[t]{2}{*}{6} & $S$ & 35.8 & 47.2 & 57.6 & 73.6 & 83.1 & 99.6 & 107.9 & 124.1 \\
\hline & $\mathrm{R}$ & 35.8 & 31.1 & 27.7 & 27.2 & 24.7 & 24.2 & 22.3 & 21.9 \\
\hline \multirow[t]{2}{*}{8} & $S$ & 38.2 & 51.2 & 63.2 & 74.6 & 92.0 & 102.7 & 120.1 & 129.6 \\
\hline & $\mathrm{R}$ & 38.2 & 33.7 & 30.4 & 27.7 & 27.3 & 25.2 & 24.7 & 23.0 \\
\hline \multirow[t]{2}{*}{10} & $S$ & 44.9 & 60.2 & 74.0 & 87.0 & 99.6 & 111.8 & 130.6 & 141.6 \\
\hline & $\mathrm{R}$ & 44.9 & 39.5 & 35.4 & 32.1 & 29.4 & 27.1 & 26.6 & 24.8 \\
\hline \multirow[t]{2}{*}{12} & $S$ & 45.6 & 61.9 & 76.8 & 91.1 & 105.0 & 125.8 & 138.4 & 159.3 \\
\hline & $\mathrm{R}$ & 45.6 & 40.6 & 36.6 & 33.4 & 30.8 & 30.2 & 28.0 & 27.4 \\
\hline \multirow[t]{2}{*}{14} & $S$ & 48.8 & 66.3 & 82.2 & 97.4 & 112.2 & 134.5 & 147.8 & 170.2 \\
\hline & $\mathrm{R}$ & 48.8 & 43.3 & 39.0 & 35.6 & 32.7 & 32.0 & 29.7 & 29.0 \\
\hline \multirow[t]{2}{*}{16} & $S$ & 48.7 & 67.0 & 84.0 & 107.5 & 123.7 & 139.2 & 162.6 & 187.4 \\
\hline & $\mathrm{R}$ & 48.7 & 43.8 & 39.8 & 38.9 & 35.7 & 33.0 & 32.2 & 31.4 \\
\hline \multirow[t]{2}{*}{18} & S & 53.2 & 73.2 & 85.4 & 109.3 & $134.9^{\circ}$ & 151.7 & 177.3 & 204.4 \\
\hline & $\mathrm{R}$ & 53.2 & 47.7 & 40.5 & 39.6 & 38.6 & 35.6 & 34.7 & 33.7 \\
\hline
\end{tabular}

Note: Energy Cost $=\$ 11.00 / \mathrm{MBtu}$

Return Pipe Temperature $=150 \mathrm{~F}$ 
TABLE $A-9 \cdot b$

\section{Economic Insulation Thickness (inch) for Hot Water Supply and Return Pipes for Energy Cost of $\$ 11 /$ MBtu}

$\begin{array}{lllllllll}\begin{array}{c}\text { Pipe } \\ \text { Size } \\ \text { (inch) }\end{array} & 150 & \underline{7} \text { Proces } & \text { Fluid } & \text { Temperature (F) } \\ 1 & 2.0 & 2.0 & 2.5 & 2.5 & 3.0 & 3.0 & 3.0 & 3.0 \\ 2 & 2.5 & 2.5 & 3.0 & 3.0 & 3.0 & 3.0 & 3.0 & 3.5 \\ 3 & 2.5 & 2.5 & 3.0 & 3.0 & 3.0 & 3.0 & 3.5 & 3.5 \\ 4 & 2.5 & 2.5 & 3.5 & 3.5 & 3.5 & 3.5 & 3.5 & 3.5 \\ 5 & 2.5 & 3.0 & 3.5 & 3.5 & 4.0 & 4.0 & 4.0 & 4.0 \\ 6 & 2.5 & 3.0 & 3.5 & 3.5 & 4.0 & 4.0 & 4.5 & 4.5 \\ 8 & 3.0 & 3.5 & 4.0 & 4.5 & 4.5 & 5.0 & 5.0 & 5.5 \\ 10 & -3.0 & 3.5 & 4.0 & 4.5 & 5.0 & 5.5 & 5.5 & 6.0 \\ 12 & 3.5 & 4.0 & 4.5 & 5.0 & 5.5 & 5.5 & 6.0 & 6.0 \\ 14 & 3.5 & 4.0 & 4.5 & 5.0 & 5.5 & 5.5 & 6.0 & 6.0 \\ 16 & 4.0 & 4.5 & 5.0 & 5.0 & 5.5 & 6.0 & 6.0 & 6.0 \\ 18 & 4.0 & 4.5 & 5.5 & 5.5 & 5.5 & 6.0 & 6.0 & 6.0\end{array}$

Note: Energy Cost $=\$ 11.00 / \mathrm{MBtu}$

Return Pipe Temperature $=150 \mathrm{~F}$ 
TABLE A-10.a

Maximum Allowable Heat Losses (Btu/h.ft) from the Supply

Pipe (S) and the Return Pipe (R) for Energy Cost of $\$ 12 / M$ Btu

\begin{tabular}{|c|c|c|c|c|c|c|c|c|c|}
\hline \multirow{2}{*}{$\begin{array}{l}\text { Pipe } \\
\text { Size } \\
\text { (inch) }\end{array}$} & \multirow[b]{2}{*}{ Pipe } & \multicolumn{8}{|c|}{ Process Fluid Temperature (F) } \\
\hline & & $\underline{150}$ & $\underline{200}$ & $\underline{250}$ & $\underline{300}$ & $\underline{350}$ & $\underline{400}$ & $\underline{450}$ & $\underline{500}$ \\
\hline \multirow[t]{2}{*}{1} & $\mathrm{~S}$ & 15.1 & 20.1 & 27.0 & 31.6 & 39.0 & 46.7 & 54.6 & 62.7 \\
\hline & $\mathbf{R}$ & 15.1 & 13.4 & 13.2 & 12.1 & 11.9 & 11.8 & 11.6 & 11.5 \\
\hline \multirow[t]{2}{*}{2} & $S$ & 18.5 & 27.5 & 33.5 & 42.8 & 52.8 & 63.2 & 68.1 & 78.2 \\
\hline & $\mathrm{R}$ & 18.5 & 18.3 & 16.4 & 16.1 & 15.9 & 15.7 & 14.4 & 14.2 \\
\hline \multirow[t]{2}{*}{3} & $S$ & 23.3 & 31.1 & 42.0 & 53.6 & 66.2 & 72.4 & 84.6 & 90.1 \\
\hline & $R$ & 23.3 & 20.7 & 20.4 & 20.1 & 19.7 & 17.4 & 17.6 & 16.2 \\
\hline \multirow[t]{2}{*}{4} & $S$ & 27.4 & 36.4 & 44.7 & 57.1 & 70.4 & 84.4 & 98.6 & 104.5 \\
\hline & $\mathrm{R}$ & 27.4 & 24.1 & 21.7 & 21.4 & 21.0 & 20.7 & 20.3 & 18.6 \\
\hline \multirow[t]{2}{*}{5} & $S$ & 31.7 & 41.9 & 51.2 & 65.4 & 74.1 & 88.8 & 103.8 & 119.4 \\
\hline & $\mathrm{R}$ & 31.7 & 27.7 & 24.7 & 24.3 & 22.2 & 21.8 & 21.4 & 21.0 \\
\hline \multirow[t]{2}{*}{6} & $S$ & 35.8 & 47.2 & 57.6 & 67.4 & 83.1 & 92.3 & 107.9 & 116.0 \\
\hline & R & 35.8 & 31.1 & 27.7 & 25.1 & 24.7 & 22.7 & 22.3 & 20.7 \\
\hline \multirow[t]{2}{*}{8} & $S$ & 38.2 & 51.2 & 63.2 & 74.6 & 92.0 & 102.7 & 120.1 & 129.6 \\
\hline & $\mathrm{R}$ & 38.2 & 33.7 & 30.4 & 27.7 & 27.3 & 25.2 & 24.7 & 23.0 \\
\hline \multirow[t]{2}{*}{10} & $S$ & 40.3 & 54.8 & 68.0 & 80.8 & 99.6 & 111.8 & 130.6 & 141.6 \\
\hline & $\mathrm{R}$ & 40.3 & 36.1 & 32.7 & 29.9 & 29.4 & 27.1 & 26.6 & 24.8 \\
\hline \multirow[t]{2}{*}{12} & $S$ & 41.1 & 56.8 & 71.2 & 91.1 & 105.0 & 118.4 & 138.4 & 159.3 \\
\hline & $\mathrm{R}$ & 41.1 & 37.3 & 34.1 & 33.4 & 30.8 & 28.6 & 28.0 & 27.4 \\
\hline \multirow[t]{2}{*}{14} & $S$ & 44.2 & 60.8 & 76.1 & 97.4 & 112.2 & 126.4 & 147.8 & 170.2 \\
\hline & $\mathrm{R}$ & 44.2 & 39.9 & 36.3 & 35.6 & 32.7 & 30.3 & 29.7 & 29.0 \\
\hline \multirow[t]{2}{*}{16} & $S$ & 48.7 & 67.0 & 84.0 & 100.2 & 123.7 & 139.2 & 162.6 & 187.4 \\
\hline & R & 48.7 & 43.8 & 39.8 & 36.5 & 35.7 & 33.0 & 32.2 & 31.4 \\
\hline \multirow[t]{2}{*}{18} & $S$ & 53.2 & 67.7 & 85.4 & 109.3 & 134.9 & 151.7 & 177.3 & 204.4 \\
\hline & R & 53.2 & 44.2 & 40.5 & 39.6 & 38.6 & 35.6 & 34.7 & 33.7 \\
\hline
\end{tabular}

Note: Energy Cost $=\$ 12.00 / \mathrm{MBtu}$

Return Pipe Temperature $=150 \mathrm{~F}$ 
TABLE $\mathrm{A}-10 \cdot \mathrm{b}$

Economic Insulation Thickness (inch) for Hot Water Supply and Return Pipes for Energy Cost of $\$ 12 / \mathrm{MBtu}$

\begin{tabular}{ccccccccc}
$\begin{array}{c}\text { Pipe } \\
\text { Size } \\
\text { (inch) }\end{array}$ & \multicolumn{7}{c}{ Process Fluid Temperature (F) } \\
\cline { 2 - 8 } 1 & 2.0 & 2.5 & 2.5 & 3.0 & 3.0 & 3.0 & 3.0 & 3.0 \\
2 & 2.5 & 2.5 & 3.0 & 3.0 & 3.0 & 3.0 & 3.5 & 3.5 \\
3 & 2.5 & 3.0 & 3.0 & 3.0 & 3.0 & 3.5 & 3.5 & 4.0 \\
4 & 2.5 & 3.0 & 3.5 & 3.5 & 3.5 & 3.5 & 3.5 & 4.0 \\
5 & 2.5 & 3.0 & 3.5 & 3.5 & 4.0 & 4.0 & 4.0 & 4.0 \\
6 & 2.5 & 3.0 & 3.5 & 4.0 & 4.0 & 4.5 & 4.5 & 5.0 \\
8 & 3.0 & 3.5 & 4.0 & 4.5 & 4.5 & 5.0 & 5.0 & 5.5 \\
10 & 3.5 & 4.0 & 4.5 & 5.0 & 5.0 & 5.5 & 5.5 & 6.0 \\
12 & 4.0 & 4.5 & 5.0 & 5.0 & 5.5 & 6.0 & 6.0 & 6.0 \\
14 & 4.0 & 4.5 & 5.0 & 5.0 & 5.5 & 6.0 & 6.0 & 6.0 \\
16 & 4.0 & 4.5 & 5.0 & 5.5 & 5.5 & 6.0 & 6.0 & 6.0 \\
18 & 4.5 & 5.0 & 5.5 & 5.5 & 5.5 & 6.0 & 6.0 & 6.0
\end{tabular}

Note: Energy Cost $=\$ 12.00 / \mathrm{MBtu}$

Return Pipe Temperature $=150 \mathrm{~F}$ 
TABLE A-11.a

Maximum Allowable Heat Losses (Btu/h.ft) from the Supply Pipe (S) and the Return Pipe (R) for Energy Cost of $\$ 13 /$ MBtu

\begin{tabular}{|c|c|c|c|c|c|c|c|c|c|}
\hline \multirow{3}{*}{$\begin{array}{c}\text { Pipe } \\
\text { Size } \\
\text { (inch) }\end{array}$} & \multirow[b]{2}{*}{ Pipe } & \multicolumn{8}{|c|}{ Process Fluid Temperature (F) } \\
\hline & & & & & & & & & \\
\hline & Type & $\underline{150}$ & $\underline{200}$ & $\underline{250}$ & $\underline{300}$ & $\underline{350}$ & $\underline{400}$ & $\underline{450}$ & $\underline{500}$ \\
\hline \multirow[t]{2}{*}{1} & $S$ & 15.1 & 20.1 & 24.8 & 31.6 & 39.0 & 46.7 & 54.6 & 62.7 \\
\hline & $\mathrm{R}$ & 15.1 & 13.4 & 12.2 & 12.1 & 11.9 & .11 .8 & 11.6 & 11.5 \\
\hline \multirow[t]{2}{*}{2} & S & 18.5 & 24.9 & 33.5 & 42.8 & 52.8 & 58.2 & 68.1 & 78.2 \\
\hline & $\mathrm{R}$ & 18.5 & 16.6 & 16.4 & 16.1 & 15.9 & 14.6 & 14.4 & 14.2 \\
\hline \multirow[t]{2}{*}{3} & $S$ & 20.9 & 31.1 & 42.0 & 53.6 & 66.2 & 72.4 & 84.6 & 90.1 \\
\hline & $\mathrm{R}$ & 20.9 & 20.7 & 20.4 & 20.1 & 19.7 & 17.9 & 17.6 & 16.2 \\
\hline \multirow[t]{2}{*}{4} & $S$ & 24.5 & 36.4 & 44.7 & 57.1 & 70.4 & 84.4 & 98.6 & 104.5 \\
\hline & $\mathrm{R}$ & 24.5 & 24.1 & 21.7 & 21.4 & 21.0 & 20.7 & 20.3 & 18.6 \\
\hline \multirow[t]{2}{*}{5} & $S$ & 28.1 & 41.9 & 51.2 & 60.1 & 74.1 & 88.8 & 103.8 & 119.4 \\
\hline & $\mathrm{R}$ & 28.1 & 27.7 & 24.7 & 22.5 & 22.2 & 21.8 & 21.4 & 21.0 \\
\hline \multirow[t]{2}{*}{6} & $S$ & 31.7 & 42.6 & 57.6 & 67.4 & 77.1 & 92.3 & 107.9 & 116.0 \\
\hline & $\mathrm{R}$ & 31.7 & 28.2 & 27.7 & 25.1 & 23.1 & 22.7 & 22.3 & 20.7 \\
\hline \multirow[t]{2}{*}{8} & $S$ & 38.2 & 51.2 & 63.2 & 74.6 & 85.7 & 102.7 & 112.7 & 129.6 \\
\hline & $\mathrm{R}$ & 38.2 & 33.7 & 30.4 & 27.7 & 25.6 & 25.2 & 23.4 & 23.0 \\
\hline \multirow[t]{2}{*}{10} & $S$ & 40.3 & 54.8 & 68.0 & 80.8 & 93.3 & 111.8 & 123.1 & 141.6 \\
\hline & $\mathrm{R}$ & 40.3 & 36.1 & 32.7 & 29.9 & 27.6 & 27.1 & 25.3 & 24.8 \\
\hline \multirow[t]{2}{*}{12} & $S$ & 41.4 & 56.8 & 71.2 & 85.1 & 105.0 & 118.4 & 138.4 & 159.3 \\
\hline & $\mathbf{R}$ & 41.4 & 37.3 & 34.1 & 31.4 & 30.8 & 28.6 & 28.0 & 27.4 \\
\hline \multirow[t]{2}{*}{14} & $S$ & 44.2 & 60.8 & 76.1 & 91.0 & 105.5 & 126.4 & 147.8 & 170.2 \\
\hline & R & 44.2 & 39.9 & 36.3 & 33.4 & 30.9 & 30.3 & 29.7 & 29.0 \\
\hline \multirow[t]{2}{*}{16} & $S$ & 44.7 & 62.0 & 78.3 & 100.2 & 116.1 & 139.2 & 162.6 & 187.4 \\
\hline & R & 44.7 & 40.7 & 37.3 & 36.5 & 33.8 & 33.0 & 32.2 & 31.4 \\
\hline \multirow[t]{2}{*}{18} & $S$ & 48.8 & 67.7 & 85.4 & 109.3 & 126.6 & 151.7 & 177.3 & 204.4 \\
\hline & $\mathrm{R}$ & 48.8 & 44.2 & 40.5 & 39.6 & 36.5 & 35.6 & 34.7 & 33.7 \\
\hline
\end{tabular}


TABLE A-11.b

Economic Insulation Thickness (inch) for Hot Water Supply and Return Pipes for Energy Cost of $\$ 13 / \mathrm{MBtu}$

\begin{tabular}{|c|c|c|c|c|c|c|c|c|}
\hline Pipe & & & Proce & Eluid & npera & (E) & & \\
\hline (inch) & $\underline{150}$ & $\underline{200}$ & $\underline{250}$ & $\underline{300}$ & 350 & 400 & 450 & 500 \\
\hline 1 & 2.0 & 2.5 & 3.0 & 3.0 & 3.0 & 3.0 & 3.0 & 3.0 \\
\hline 2 & 2.5 & 3.0 & 3.0 & 3.0 & 3.0 & 3.5 & 3.5 & 3.5 \\
\hline 3 & 3.0 & 3.0 & 3.0 & 3.0 & 3.0 & 3.5 & 3.5 & 4.0 \\
\hline 4 & 3.0 & 3.0 & 3.5 & 3.5 & 3.5 & 3.5 & 3.5 & 4.0 \\
\hline 5 & 3.0 & 3.0 & 3.5 & 4.0 & 4.0 & 4.0 & 4.0 & 4.0 \\
\hline 6 & 3.0 & 3.5 & 3.5 & 4.0 & 4.5 & 4.5 & 4.5 & 5.0 \\
\hline 8 & 3.0 & 3.5 & 4.0 & 4.5 & 5.0 & 5.0 & 5.5 & 5.5 \\
\hline 10 & 3.5 & 4.0 & 4.5 & 5.0 & 5.5 & 5.5 & 6.0 & 6.0 \\
\hline 12 & 4.0 & 4.5 & .5 .0 & 5.5 & 5.5 & 6.0 & 6.0 & 6.0 \\
\hline 14 & 4.0 & 4.5 & 5.0 & 5.5 & 6.0 & 6.0 & 6.0 & 6.0 \\
\hline 16 & 4.5 & 5.0 & 5.5 & 5.5 & 6.0 & 6.0 & 6.0 & 6.0 \\
\hline 18 & 4.5 & 5.0 & 5.5 & 5.5 & 6.0 & 6.0 & 6.0 & 6.0 \\
\hline
\end{tabular}

Note: Energy Cost $=\$ 13.00 / \mathrm{MBtu}$

Return Pipe Temperature $=150 \mathrm{~F}$ 
TABLE A-12.a

Maximum Allowable Heat Losses (Btu/h.ft) from the Supply

Pipe ( $S$ ) and the Return Pipe (R) for Energy Cost of $\$ 14 / \mathrm{MBtu}$

\begin{tabular}{|c|c|c|c|c|c|c|c|c|c|}
\hline \multirow{3}{*}{$\begin{array}{l}\text { Pipe } \\
\text { Size } \\
\text { (inch) } \\
\end{array}$} & \multirow[b]{2}{*}{ Pipe } & \multicolumn{8}{|c|}{ Process Fluid Temperature (F) } \\
\hline & & & & & & & & & \\
\hline & Type & $\underline{150}$ & $\underline{200}$ & $\underline{250}$ & $\underline{300}$ & $\underline{350}$ & 400 & $\underline{450}$ & $\underline{500}$ \\
\hline \multirow[t]{2}{*}{1} & $S$ & 15.1 & 20.1 & 24.8 & 31.6 & 39.0 & 46.7 & 54.6 & 62.7 \\
\hline & R & 15.1 & 13.4 & 12.2 & 12.1 & 11.9 & 11.8 & 11.6 & 11.5 \\
\hline \multirow[t]{2}{*}{2} & S & 18.5 & 24.9 & 33.5 & 42.8 & 52.8 & 58.2 & 68.1 & 78.2 \\
\hline & $\mathrm{R}$ & 18.5 & 16.6 & 16.4 & 16.1 & 15.9 & 14.6 & 14.4 & 14.2 \\
\hline \multirow[t]{2}{*}{3} & $S$ & 20.9 & 31.1 & 42.0 & 53.6 & 60.4 & 67.1 & 78.4 & 90.1 \\
\hline & $\mathrm{R}$ & 20.9 & 20.7 & 20.4 & 20.1 & 18.2 & 16.7 & 16.5 & 16.2 \\
\hline \multirow[t]{2}{*}{4} & $S$ & 24.5 & 36.4 & 44.7 & 57.1 & 70.4 & 77.8 & 90.9 & 104.5 \\
\hline & $\mathrm{R}$ & 24.5 & 24.1 & 21.7 & 21.4 & 21.0 & 19.2 & 18.9 & 18.6 \\
\hline \multirow[t]{2}{*}{5} & $S$ & 28.1 & 41.9 & 51.2 & 60.1 & 74.1 & 88.8 & 103.8 & 119.4 \\
\hline & $\mathrm{R}$ & 28.1 & 27.7 & 24.7 & 22.5 & 22.2 & 21.8 & 21.4 & 21.0 \\
\hline \multirow[t]{2}{*}{6} & $S$ & 31.7 & 42.6 & 52.7 & 62.5 & 77.1 & 92.3 & 107.9 & 116.0 \\
\hline & $\mathrm{R}$ & 31.7 & 28.2 & 25.5 & 23.4 & 23.1 & 22.7 & 22.3 & 20.7 \\
\hline \multirow[t]{2}{*}{8} & S & 34.3 & 46.8 & 58.3 & $69^{\circ} .5$ & 85.7 & 96.4 & 112.7 & 122.4 \\
\hline & $\mathrm{R}$ & 34.3 & 30.9 & 28.2 & 26.0 & 25.6 & 23.8 & 23.4 & 21.8 \\
\hline \multirow[t]{2}{*}{10} & $S$ & 36.7 & 50.4 & 63.2 & 75.6 & 93.3 & 105.3 & 123.1 & 141.6 \\
\hline & $\mathrm{R}$ & 36.7 & 33.2 & 30.4 & 28.1 & 27.6 & 25.7 & 25.3 & 24.8 \\
\hline \multirow[t]{2}{*}{12} & $S$ & 38.0 & 52.7 & 66.5 & 85.1 & 98.8 & 118.4 & 138.4 & 159.3 \\
\hline & $\mathrm{R}$ & 38.0 & 34.7 & 32.0 & 31.4 & 29.1 & 28.6 & 28.0 & 27.4 \\
\hline \multirow[t]{2}{*}{14} & $S$ & 40.6 & 56.3 & 71.1 & 91.0 & 105.5 & 126.4 & 147.8 & 170.2 \\
\hline & $\mathrm{R}$ & 40.6 & 37.0 & 34.1 & 33.4 & 30.9 & 30.3 & 29.7 & 29.0 \\
\hline \multirow[t]{2}{*}{16} & $S$ & 44.7 & 62.0 & 78.3 & 94.1 & 116.1 & 139.2 & 162.6 & 187.4 \\
\hline & $\mathrm{R}$ & 44.7 & 40.7 & 37.3 & 34.5 & 33.8 & 33.0 & 32.2 & 31.4 \\
\hline \multirow[t]{2}{*}{18} & $\begin{array}{l}S \\
R\end{array}$ & 48.8 & 67.7 & 85.4 & 102.6 & 126.6 & 151.7 & 177.3 & 204.4 \\
\hline & R & 48.8 & 44.2 & 40.5 & 37.3 & 36.5 & 35.6 & 34.7 & 33.7 \\
\hline
\end{tabular}

Note: Energy Cost $=\$ 14.00 / \mathrm{MBtu}$

Return Pipe Temperature $=150 \mathrm{~F}$ 
TABLE $A-12 \cdot b$

Economic Insulation Thickness (inch) for Hot Water Supply
and Return Pipes for Energy Cost of $\$ 14 / \mathrm{MB}$ tu

\begin{tabular}{|c|c|c|c|c|c|c|c|c|}
\hline Pipe & & & roc & Elui & nper & ( & & \\
\hline $\begin{array}{c}\text { Size } \\
\text { (inch) } \\
\end{array}$ & 150 & $\underline{200}$ & $\underline{250}$ & 300 & 350 & 400 & 450 & $\underline{500}$ \\
\hline 1 & 2.0 & 2.5 & 3.0 & 3.0 & 3.0 & 3.0 & 3.0 & 3.0 \\
\hline 2 & 2.5 & 3.0 & 3.0 & 3.0 & 3.0 & 3.5 & 3.5 & 3.5 \\
\hline 3 & 3.0 & 3.0 & 3.0 & 3.0 & 3.5 & 4.0 & 4.0 & 4.0 \\
\hline 4 & 3.0 & 3.0 & 3.5 & 3.5 & 3.5 & 4.0 & 4.0 & 4.0 \\
\hline 5 & 3.0 & 3.0 & 3.5 & 4.0 & 4.0 & 4.0 & 4.0 & 4.0 \\
\hline 6 & 3.0 & 3.5 & 4.0 & 4.5 & 4.5 & 4.5 & 4.5 & 5.0 \\
\hline 8 & 3.5 & 4.0 & 4.5 & 5.0 & 5.0 & 5.5 & 5.5 & 6.0 \\
\hline 10 & -4.0 & 4.5 & 5.0 & 5.5 & 5.5 & 6.0 & 6.0 & 6.0 \\
\hline 12 & 4.5 & 5.0 & 5.5 & 5.5 & 6.0 & 6.0 & 6.0 & 6.0 \\
\hline 14 & 4.5 & 5.0 & 5.5 & 5.5 & 6.0 & 6.0 & 6.0 & 6.0 \\
\hline 16 & 4.5 & 5.0 & 5.5 & 6.0 & 6.0 & 6.0 & 6.0 & 6.0 \\
\hline 18 & 4.5 & $5.0^{\circ}$ & 5.5 & 6.0 & 6.0 & 6.0 & 6.0 & 6.0 \\
\hline
\end{tabular}

Note: Energy Cost $=\$ 14.00 / \mathrm{MBtu}$

Return Pipe Temperature $=150 \mathrm{~F}$ 
TABLE A-13.a

Maximum Allowable Heat Losses (Btu/h.ft) from the Supply Pipe (S) and the Return Pipe (R) for Energy Cost of $\$ 15 /$ MBtu

\begin{tabular}{|c|c|c|c|c|c|c|c|c|c|}
\hline \multirow{2}{*}{$\begin{array}{c}\text { Pipe } \\
\text { Size } \\
\text { (inch) } \\
\end{array}$} & \multirow[b]{2}{*}{ Pipe } & \multicolumn{8}{|c|}{ Process Fluid Temperature (F) } \\
\hline & & $\underline{150}$ & $\underline{200}$ & $\underline{250}$ & $\underline{300}$ & $\underline{350}$ & $\underline{400}$ & 450 & $\underline{500}$ \\
\hline \multirow[t]{2}{*}{1} & s & 13.5 & 20.1 & 24.8 & 31.6 & 39.0 & 46.7 & 54.6 & 62.7 \\
\hline & $\mathrm{R}$ & 13.5 & 13.4 & 12.2 & 12.1 & 11.9 & 11.8 & 11.6 & 11.5 \\
\hline \multirow[t]{2}{*}{2} & $S$ & 18.5 & 24.9 & 33.5 & 42.8 & 48.6 & 58.2 & 68.1 & 78.2 \\
\hline & $\mathrm{R}$ & 18.5 & 16.6 & 16.4 & 16.1 & 14.8 & .14 .6 & 14.4 & 14.2 \\
\hline \multirow[t]{2}{*}{3} & S & 20.9 & 31.1 & 42.0 & 53.6 & 60.4 & 67.1 & 78.4 & 90.1 \\
\hline & R & 20.9 & 20.7 & 20.4 & 20.1 & 18.2 & 16.7 & 16.5 & 16.2 \\
\hline \multirow[t]{2}{*}{4} & $S$ & 24.5 & 36.4 & 44.7 & 57.1 & 70.4 & 77.8 & 90.9 & 104.5 \\
\hline & $\mathrm{R}$ & 24.5 & 24.1 & 21.7 & 21.4 & 21.0 & 19.2 & 18.9 & 18.6 \\
\hline \multirow[t]{2}{*}{5} & S & 28.1 & 41.9 & 51.2 & 60.1 & 74.1 & 88.8 & 103.8 & 119.4 \\
\hline & $\mathrm{R}$ & 28.1 & 27.7 & 24.7 & 22.5 & 22.2 & 21.8 & 21.4 & 21.0 \\
\hline \multirow[t]{2}{*}{6} & $S$ & 31.7 & 42.6 & 52.7 & 62.5 & 77.1 & 92.3 & 107.9 & 116.0 \\
\hline & $\mathrm{R}$ & 31.7 & 28.2 & 25.5 & 23.4 & 23.1 & 22.7 & 22.3 & 20.7 \\
\hline \multirow[t]{2}{*}{8} & $S$ & 34.3 & 46.8 & 58.3 & 69.5 & 85.7 & 96.4 & 112.7 & 122.4 \\
\hline & $\mathrm{R}$ & 34.3 & 30.9 & 28.2 & 26.0 & 25.6 & 23.8 & 23.4 & 21.8 \\
\hline \multirow[t]{2}{*}{10} & S & 36.7 & 50.4 & 63.2 & 75.6 & 93.3 & 105.3 & 123.1 & 141.6 \\
\hline & $\mathrm{R}$ & 36.7 & 33.2 & 30.4 & 28.1 & 27.6 & 25.7 & 25.3 & 24.8 \\
\hline \multirow[t]{2}{*}{12} & s & 38.0 & 52.7 & 66.5 & 85.1 & 98.8 & 118.4 & 138.4 & 159.3 \\
\hline & $\mathrm{R}$ & 38.0 & 34.7 & 32.0 & 31.4 & 29.1 & 28.6 & 28.0 & 27.4 \\
\hline \multirow[t]{2}{*}{14} & $S$ & 40.6 & 56.3 & 71.1 & 91.0 & 105.5 & 126.4 & 147.8 & 170.2 \\
\hline & $\mathrm{R}$ & 40.6 & 37.0 & 34.1 & 33.4 & 30.9 & 30.3 & 29.7 & 29.0 \\
\hline \multirow[t]{2}{*}{16} & $S$ & 44.7 & 62.0 & 78.3 & 94.1 & 116.1 & 139.2 & 162.6 & 187.4 \\
\hline & $R$ & 44.7 & 40.7 & 37.3 & 34.5 & 33.8 & 33.0 & 32.2 & 31.4 \\
\hline \multirow[t]{2}{*}{18} & $S$ & 48.8 & 67.7 & 85.4 & 102.6 & 126.6 & 151.7 & 177.3 & 204.4 \\
\hline & $\mathrm{R}$ & 48.8 & 44.2 & 40.5 & 37.3 & 36.5 & 35.6 & 34.7 & 33.7 \\
\hline
\end{tabular}

Note: Energy Cost $=\$ 15.00 / \mathrm{MBtu}$

Return Pipe Temperature $=150 \mathrm{~F}$ 
TABLE $A-13 . b$

Economic Insulation Thickness (inch) for Hot Water Supply and Return Pipes for Energy Cost of $\$ 15 / \mathrm{MBtu}$

$\begin{array}{ccccccccc}\begin{array}{c}\text { Pipe } \\ \begin{array}{c}\text { Size } \\ \text { (inch) }\end{array}\end{array} & \begin{array}{l}150 \\$\cline { 3 - 6 }\end{array} & $\underline{200} & \underline{250} & \underline{300} & \underline{350} & \underline{400} & \underline{450} & \underline{500} \\ 1 & 2.5 & 2.5 & 3.0 & 3.0 & 3.0 & 3.0 & 3.0 & 3.0 \\ 2 & 2.5 & 3.0 & 3.0 & 3.0 & 3.5 & 3.5 & 3.5 & 3.5 \\ 3 & 3.0 & 3.0 & 3.0 & 3.0 & 3.5 & 4.0 & 4.0 & 4.0 \\ 4 & 3.0 & 3.0 & 3.5 & 3.5 & 3.5 & 4.0 & 4.0 & 4.0 \\ 5 & 3.0 & 3.0 & 3.5 & 4.0 & 4.0 & 4.0 & 4.0 & 4.0 \\ 6 & 3.0 & 3.5 & 4.0 & 4.5 & 4.5 & 4.5 & 4.5 & 5.0 \\ 8 & 3.5 & 4.0 & 4.5 & 5.0 & 5.0 & 5.5 & 5.5 & 6.0 \\ 10 & 4.0 & 4.5 & 5.0 & 5.5 & 5.5 & 6.0 & 6.0 & 6.0 \\ 12 & 4.5 & 5.0 & 5.5 & 5.5 & 6.0 & 6.0 & 6.0 & 6.0 \\ 14 & 4.5 & 5.0 & 5.5 & 5.5 & 6.0 & 6.0 & 6.0 & 6.0 \\ 16 & 4.5 & 5.0 & 5.5 & 6.0 & 6.0 & 6.0 & 6.0 & 6.0 \\ 18 & 4.5 & 5.0 & 5.5 & 6.0 & 6.0 & 6.0 & 6.0 & 6.0\end{array}$

Note: Energy Cost $=\$ 15.00 / \mathrm{MBtu}$

Return Pipe Temperature $=150 \mathrm{~F}$ 
TABLE B-1.a

Maximum Allowable Heat Losses (Btu/h・ft) from the Supply

Pipe (S) and the Return Pipe (R) for Energy Cost of $\$ 3 / M B t u$

\begin{tabular}{|c|c|c|c|c|c|c|c|c|c|}
\hline \multirow{2}{*}{$\begin{array}{c}\text { Pipe } \\
\text { Size } \\
\text { (inch) }\end{array}$} & \multirow[b]{2}{*}{ Pipe } & \multicolumn{8}{|c|}{ Process Fluid Temperature (F) } \\
\hline & & $\underline{150}$ & $\underline{200}$ & $\underline{250}$ & 300 & 350 & $\underline{400}$ & $\underline{450}$ & $\underline{500}$ \\
\hline 2 & S & 25.3 & 37.6 & 50.7 & 31.6 & 64.8 & 95.7 & 93.4 & 107.4 \\
\hline 1 & R & 17.4 & 17.1 & 16.7 & 16.4 & 16.0 & 15.6 & 13.4 & 13.1 \\
\hline 3 & $S$ & 32.8 & 48.8 & 65.9 & 84.3 & 85.6 & 102.5 & 119.8 & 137.9 \\
\hline 1.5 & $\mathrm{R}$ & 21.5 & 21.0 & 20.4 & 19.8 & 16.7 & 16.3 & 15.9 & 15.4 \\
\hline 4 & $S$ & 39.1 & 58.4 & 79.0 & 101.0 & 101.7 & 104.8 & 122.4 & 141.0 \\
\hline 2 & $\mathrm{R}$ & 24.5 & 23.9 & 23.1 & 22.4 & 18.7 & 16.4 & 16.0 & 15.6 \\
\hline 5 & S- & 45.7 & 68.3 & 92.4 & 96.1 & 118.5 & 121.4 & 141.8 & 163.3 \\
\hline 2.5 & R & 27.6 & 26.8 & 25.8 & 21.4 & 20.7 & 18.1 & 17.6 & 17.0 \\
\hline 6 & $S$ & 52.1 & 78.0 & 105.6 & 109.4 & 134.8 & 137.6 & 160.7 & 185.2 \\
\hline 3 & $\mathrm{R}$ & 31.5 & 30.4 & 29.2 & 24.0 & 23.1 & 20.1 & 19.4 & 18.7 \\
\hline 8 & $S$ & 63.7 & 95.8 & 104.6 & 133.9 & 139.8 & 167.5 & 195.6 & 225.7 \\
\hline 4 & $\mathrm{R}$ & 37.3 & 35.7 & 28.9 & 27.8 & 23.8 & 23.0 & 22.1 & 21.1 \\
\hline 10 & $S$ & 75.2 & 113.6 & $124: 1$ & 134.3 & 165.6 & 198.4 & 201.2 & 233.3 \\
\hline 6 & $\mathrm{R}$ & 49.5 & 47.2 & 37.8 & 31.9 & 30.7 & 29.4 & 25.9 & 24.9 \\
\hline 12 & $S$ & 86.7 & 130.8 & 142.6 & 153.9 & 189.8 & 227.4 & 231.1 & 266.8 \\
\hline 6 & $\mathrm{R}$ & 48.9 & 46.3 & 37.0 & 31.2 & 29.8 & 28.4 & 25.1 & 23.9 \\
\hline 14 & $S$ & 92.7 & 113.1 & 153.5 & 165.7 & 204.4 & 245.0 & 248.8 & 254.9 \\
\hline 8 & $\mathrm{R}$ & 60.0 & 47.3 & 45.1 & 37.8 & 36.1 & 34.3 & 30.1 & 26.8 \\
\hline 16 & $S$ & 104.6 & 126.5 & 171.6 & 185.0 & 197.4 & 236.6 & 245.0 & 282.8 \\
\hline 8 & R & 59.6 & 46.6 & 44.2 & 37.0 & 31.8 & 30.3 & 26.8 & 25.5 \\
\hline 18 & S & 113.5 & 138.3 & 157.7 & 202.3 & 216.1 & 259.0 & 268.2 & 309.8 \\
\hline 10 & $\mathrm{R}$ & 70.4 & .54 .7 & 45.1 & 42.7 & 36.6 & 34.7 & 30.6 & 29.0 \\
\hline
\end{tabular}


TABLE B-1.b

Economic Insulation Thickness (inch) for Steam Supply and Return Pipes for Energy Cost of $\$ 3 / \mathrm{MBtu}$

$\begin{array}{ccccccccc}\begin{array}{c}\text { Pipe } \\ \begin{array}{c}\text { Size } \\ \text { (inch) }\end{array}\end{array} & \underline{150} & \underline{200} & \underline{250} & \underline{300} & \underline{350} & \underline{400} & \underline{40} & \underline{500} \\ 2 \& 1 & 1.5 & 1.5 & 1.5 & 1.5 & 1.5 & 1.5 & 2.0 & 2.0 \\ 3 \& 1.5 & 1.5 & 1.5 & 1.5 & 1.5 & 2.0 & 2.0 & 2.0 & 2.0 \\ 4 \& 2 & 1.5 & 1.5 & 1.5 & 1.5 & 2.0 & 2.5 & 2.5 & 2.5 \\ 5 \& 2.5 & 1.5 & 1.5 & 1.5 & 1.5 & 2.0 & 2.0 & 2.5 & 2.5 \\ 6 \& 3 & 1.5 & 1.5 & 1.5 & 2.0 & 2.0 & 2.5 & 2.5 & 2.5 \\ 8 \& 4 & 1.5 & 1.5 & 2.0 & 2.0 & 2.5 & 2.5 & 2.5 & 2.5 \\ -10 \& 6 & 1.5 & 1.5 & 2.0 & 2.5 & 2.5 & 2.5 & 3.0 & 3.0 \\ 12 \& 6 & 1.5 & 1.5 & 2.0 & 2.5 & 2.5 & 2.5 & 3.0 & 3.0 \\ 14 \& 8 & 1.5 & 2.0 & 2.0 & 2.5 & 2.5 & 2.5 & 3.0 & 3.5 \\ 16 \& 8 & 1.5 & 2.0 & 2.0 & 1.5 & 3.0 & 3.0 & 3.5 & 3.5 \\ 18 \& 10 & 1.5 & 2.0 & 2.5 & 2.5 & 3.0 & 3.0 & 3.5 & 3.5\end{array}$

Note: Energy Cost $=\$ 3.00 / \mathrm{MBtu}$

Return Pipe Temperature $=150 \mathrm{~F}$ 
TABLE $B-2 \cdot a$

Maximum Allowable Heat Losses (Btu/h'ft) from the Supply

Pipe (S) and the Return Pipe (R) for Energy Cost of $\$ 4 /$ MBtu

\begin{tabular}{|c|c|c|c|c|c|c|c|c|c|}
\hline \multirow{2}{*}{$\begin{array}{c}\text { Pipe } \\
\text { Size } \\
\text { (inch) }\end{array}$} & \multirow[b]{2}{*}{$\begin{array}{l}\text { Pipe } \\
\text { Type }\end{array}$} & \multicolumn{8}{|c|}{ Process Fluid Temperature (F) } \\
\hline & & $\underline{150}$ & $\underline{200}$ & 250 & 300 & 350 & $\underline{400}$ & 450 & $\underline{500}$ \\
\hline 2 & $S$ & 25.3 & 37.6 & 50.7 & 64.8 & $66 . \dot{7}$ & 79.9 & 81.9 & 94.1 \\
\hline 1 & $\mathrm{R}$ & 17.4 & 17.1 & 16.7 & 16.4 & 14.0 & 13.7 & 12.2 & 12.0 \\
\hline 3 & $S$ & 32.8 & 48.8 & 65.9 & 69.4 & 85.6 & 88.8 & 103.8 & 119.4 \\
\hline 1.5 & $\mathrm{R}$ & 21.5 & 21.0 & 20.4 & 17.1 & 16.7 & 14.8 & 14.4 & 14.1 \\
\hline 4 & $S$ & 39.1 & 58.4 & 79.0 & 82.5 & 87.5 & 104.8 & 122.4 & 141.0 \\
\hline 2 & $\mathbf{R}$ & 24.5 & 23.9 & 23.1 & 19.3 & 16.9 & 16.4 & 16.0 & 15.6 \\
\hline 5 & $S$ & 45.7 & 68.3 & 75.1 & 96.1 & 101.3 & 121.4 & 141.8 & 144.2 \\
\hline 2.5 & $\mathrm{R}$ & 27.6 & 26.8 & 22.1 & 21.4 & 18.6 & 18.1 & 17.6 & 15.8 \\
\hline 6 & $s^{-}=$ & 52.1 & 78.0 & 85.5 & 109.4 & 114.9 & 137.6 & 141.5 & 162.9 \\
\hline 3 & $\mathrm{R}$ & 31.5 & 30.4 & 24.8 & 24.0 & 20.7 & 20.1 & 18.0 & 17.4 \\
\hline 8 & $S$ & 63.7 & 77.3 & 104.6 & 113.4 & 139.8 & 146.7 & 171.3 & 197.4 \\
\hline 4 & $\mathrm{R}$ & 37.3 & 30.0 & 28.9 & 24.7 & 23.8 & 21.1 & 20.4 & 19.7 \\
\hline 10 & $S$ & 75.2 & 91.6 & 104.9 & 134.3 & 144.5 & 173.1 & 180.5 & 208.1 \\
\hline 6 & $\mathbf{R}$ & 49.5 & 39.3 & 33.0 & 31.9 & 27.8 & 26.9 & 24.1 & 23.3 \\
\hline 12 & $S$ & 86.7 & 105.2 & 120.2 & 153.9 & 165.2 & 197.9 & 205.8 & 237.3 \\
\hline 6 & $\mathrm{R}$ & 48.9 & 38.7 & 32.5 & 31.2 & 27.2 & 26.2 & 23.5 & 22.6 \\
\hline 14 & $S$ & 92.7 & 113.1 & 129.4 & 165.7 & 177.7 & 213.0 & 220.9 & 230.1 \\
\hline 8 & $\mathrm{R}$ & 60.0 & 47.3 & 39.4 & 37.8 & 32.8 & 31.5 & 27.9 & 25.1 \\
\hline 16 & $S$ & 104.3 & 126.5 & 144.4 & 160.1 & 175.0 & 209.7 & 245.0 & 254.9 \\
\hline 8 & $R$ & 59.6 & 46.6 & 38.8 & 33.3 & 29.2 & 28.0 & 26.8 & 24.1 \\
\hline 18 & $S$ & 91.5 & 116.3 & 136.7 & 175.2 & 191.5 & 229.5 & 241.7 & 279.0 \\
\hline 10 & R & 57.6 & 47.2 & 40.2 & 38.5 & 33.7 & 32.2 & 28.8 & 27.4 \\
\hline
\end{tabular}


TABLE B-2.b

Economic Insulation Thickness (inch) for Steam Supply and Return Pipes for Energy Cost of $\$ 4 / M B t u$

\begin{tabular}{|c|c|c|c|c|c|c|c|c|}
\hline \multirow{2}{*}{$\begin{array}{l}\text { Pipe } \\
\text { Size } \\
\text { (inch) } \\
\end{array}$} & \multicolumn{8}{|c|}{ Process Fluid Temperature (F) } \\
\hline & $\underline{150}$ & $\underline{200}$ & $\underline{250}$ & 300 & $\underline{350}$ & 400 & 450 & $\underline{500}$ \\
\hline $2 \& 1$ & 1.5 & 1.5 & 1.5 & 1.5 & 2.0 & 2.0 & 2.5 & 2.5 \\
\hline $3 \& 1.5$ & 1.5 & 1.5 & 1.5 & 2.0 & 2.0 & 2.5 & 2.5 & 2.5 \\
\hline $4 \& 2$ & 1.5 & 1.5 & 1.5 & 2.0 & 2.5 & 2.5 & 2.5 & 2.5 \\
\hline $5 \& 2.5$ & 1.5 & 1.5 & 2.0 & 2.0 & 2.5 & 2.5 & 2.5 & 3.0 \\
\hline $6 \& 3$ & 1.5 & 1.5 & 2.0 & 2.0 & 2.5 & 2.5 & 3.0 & 3.0 \\
\hline $8 \& 4$ & 1.5 & 2.0 & 2.0 & 2.5 & 2.5 & 3.0 & 3.0 & 3.0 \\
\hline $10 \& 6$ & 1.5 & 2.0 & 2.5 & 2.5 & 3.0 & 3.0 & 3.5 & 3.5 \\
\hline $12 \& 6$ & -1.5 & 2.0 & 2.5 & 2.5 & 3.0 & 3.0 & 3.5 & 3.5 \\
\hline $14 \& 8$ & 1.5 & 2.0 & 2.5 & 2.5 & 3.0 & 3.0 & 3.5 & 4.0 \\
\hline $16 \& 8$ & 1.5 & 2.0 & 2.5 & 3.0 & 3.5 & 3.5 & 3.5 & 4.0 \\
\hline $18 \& 10$ & 2.0 & 2.5 & 3.0 & 3.0 & 3.5 & 3.5 & 4.0 & 4.0 \\
\hline
\end{tabular}

Note: Energy Cost $=\$ 4.00 / \mathrm{MBtu}$

Return Pipe Temperature $=150 \mathrm{~F}$ 
TABLE B-3.a

Maximum Allowable Heat Losses (Btu/h・ft) from the Supply

Pipe ( $S$ ) and the Return Pipe (R) for Energy Cost of $\$ 5 /$ MBtu

\begin{tabular}{|c|c|c|c|c|c|c|c|c|c|}
\hline \multirow{3}{*}{$\begin{array}{c}\text { Pipe } \\
\text { Size } \\
\text { (inch) }\end{array}$} & \multirow[b]{2}{*}{ Pipe } & \multicolumn{8}{|c|}{ Process Fluid Temperature (F) } \\
\hline & & & & & & & & & \\
\hline & Type & $\underline{150}$ & 200 & $\underline{250}$ & 300 & 350 & $\underline{400}$ & 450 & $\underline{500}$ \\
\hline 2 & S & 25.3 & 37.6 & 50.7 & 54.1 & 58.5 & 70.1 & 81.9 & 94.1 \\
\hline 1 & $\mathbf{R}$ & 17.4 & 17.1 & 16.7 & 14.3 & 12.7 & 12.5 & 12.2 & 12.0 \\
\hline 3 & S & 32.8 & 48.8 & 54.3 & 69.4 & 74.1 & 79.4 & 92.8 & 106.4 \\
\hline 1.5 & $\mathbf{R}$ & 21.5 & 21.0 & 17.5 & 17.1 & 15.1 & 13.6 & 13.4 & 13.1 \\
\hline 4 & $S$ & 39.1 & 58.4 & 64.5 & 71.0 & 87.5 & 93.1 & 108.8 & 125.1 \\
\hline 2 & $\mathrm{R}$ & 24.5 & $23.9^{\circ}$ & 19.8 & 17.2 & 16.9 & 15.2 & 14.8 & 14.5 \\
\hline 5 & $S$ & 45.7 & 55.6 & 75.1 & 82.2 & 101.3 & 107.3 & 125.3 & 144.2 \\
\hline 2.5 & $\mathbf{R}$ & 27.6 & 22.7 & 22.1 & 19.1 & 18.6 & 16.6 & 16.2 & 15.8 \\
\hline 6 & S & 52.1 & 63.2 & 85.5 & 93.2 & 114.9 & 121.1 & 141.5 & 162.9 \\
\hline 3 & $\mathbf{R}$ & 31.5 & 25.6 & 24.8 & 21.4 & 20.7 & 18.5 & 18.0 & 17.4 \\
\hline 8 & $S$ & 51.7 & 77.3 & 88.7 & 113.4 & 122.4 & 146.7 & 153.5 & 176.8 \\
\hline 4 & $\mathbf{R}$ & 31.0 & 30.0 & 25.5 & 24.7 & 21.8 & 21.1 & 19.1 & 18.5 \\
\hline 10 & S & 61.0 & 77.5 & 104.9 & 117.2 & 144.5 & 154.6 & 180.5 & 208.1 \\
\hline 6 & $\mathbf{R}$ & 40.8 & 34.1 & 33.0 & 28.7 & 27.8 & 24.9 & 24.1 & 23.3 \\
\hline 12 & $S$ & 70.1 & 88.9 & 120.2 & 134.0 & 147.1 & 176.2 & 205.8 & 214.7 \\
\hline 6 & $\mathbf{R}$ & 40.4 & 33.7 & 32.5 & 28.2 & 25.1 & 24.3 & 23.5 & 21.3 \\
\hline 14 & $S$ & 75.1 & 95.5 & 129.4 & 144.1 & 157.9 & 189.2 & 199.5 & 210.5 \\
\hline 8 & $\mathbf{R}$ & 49.3 & 40.9 & 39.4 & 34.1 & 30.0 & 29.0 & 26.0 & 23.7 \\
\hline 16 & $S$ & 84.0 & 106.6 & 125.1 & 141.9 & 175.0 & 189.2 & 220.9 & 232.9 \\
\hline 8 & $\mathbf{R}$ & 48.9 & 40.5 & 34.6 & 30.4 & 29.2 & 26.2 & 25.2 & 22.9 \\
\hline 18 & $S$ & 77.2 & 100.9 & 136.7 & 155.3 & 191.5 & 206.9 & 220.8 & 254.8 \\
\hline 10 & $\mathbf{R}$ & 49.2 & 41.8 & 40.2 & 35.1 & 33.7 & 30.0 & 27.2 & 26.1 \\
\hline
\end{tabular}


TABLE $B-3 \cdot b$

Economic Insulation Thickness (inch) for Steam Supply and Return Pipes for Energy Cost of $\$ 5 / \mathrm{MBtu}$

\begin{tabular}{|c|c|c|c|c|c|c|c|c|}
\hline \multirow{2}{*}{$\begin{array}{c}\text { Pipe } \\
\text { Size } \\
\text { (inch) } \\
\end{array}$} & \multicolumn{8}{|c|}{ Process Fluid Temperature (F) } \\
\hline & 150 & $\underline{200}$ & $\underline{250}$ & 300 & 350 & 400 & 450 & 500 \\
\hline $2 \& 1$ & 1.5 & 1.5 & 1.5 & 2.0 & 2.5 & 2.5 & 2.5 & 2.5 \\
\hline $3 \& 1.5$ & 1.5 & 1.5 & 2.0 & 2.0 & 2.5 & 3.0 & 3.0 & 3.0 \\
\hline $4 \& 2$ & 1.5 & 1.5 & 2.0 & 2.5 & 2.5 & 3.0 & 3.0 & 3.0 \\
\hline $5 \& 2.5$ & 1.5 & 2.0 & 2.0 & 2.5 & 2.5 & 3.0 & 3.0 & 3.0 \\
\hline $6 \& 3$ & 1.5 & 2.0 & 2.0 & 2.5 & 2.5 & 3.0 & 3.0 & 3.0 \\
\hline $8 \& 4$ & 2.0 & 2.0 & 2.5 & 2.5 & 3.0 & 3.0 & 3.5 & 3.5 \\
\hline $10 \& 6$ & 2.0 & 2.5 & 2.5 & 3.0 & 3.0 & 3.5 & 3.5 & 3.5 \\
\hline $12 \& 6$ & -2.0 & 2.5 & 2.5 & 3.0 & 3.5 & 3.5 & 3.5 & 4.0 \\
\hline $14 \& 8$ & 2.0 & 2.5 & 2.5 & 3.0 & 3.5 & 3.5 & 4.0 & 4.5 \\
\hline $16 \& 8$ & 2.0 & 2.5 & 3.0 & 3.5 & 3.5 & 4.0 & 4.0 & 4.5 \\
\hline $18 \& 10$ & 2.5 & 3.0 & 3.0 & 3.5 & 3.5 & 4.0 & 4.5 & 4.5 \\
\hline
\end{tabular}

Note: Energy Cost $=\$ 5.00 / \mathrm{MBtu}$

Return Pipe Temperature $=150 \mathrm{~F}$ 
TABLE B-4.a

Maximum Allowable heat Losses (Btu/h/ft) from the Supply

Pipe (S) and the Return Pipe (R) for Energy Cost of $\$ 6 / \mathrm{MBtu}$

\begin{tabular}{|c|c|c|c|c|c|c|c|c|c|}
\hline \multirow{3}{*}{$\begin{array}{c}\text { Pipe } \\
\text { Size } \\
\text { (inch) }\end{array}$} & \multirow[b]{2}{*}{ Pipe } & \multicolumn{8}{|c|}{ Process Fluid Temperature (F) } \\
\hline & & & & & & & & & \\
\hline & Type & $\underline{150}$ & $\underline{200}$ & $\underline{250}$ & $\underline{300}$ & 350 & 400 & $\underline{450}$ & $\underline{500}$ \\
\hline 2 & S & 25.3 & 37.6 & 42.4 & 54.1 & 58.5 & 70.1 & 81.9 & 85.0 \\
\hline 1 & $\mathrm{R}$ & 17.4 & 17.1 & 14.5 & 14.3 & 12.7 & 12.5 & 12.2 & 11.2 \\
\hline 3 & $\mathrm{~S}$ & 32.8 & 40.3 & 54.3 & 60.1 & 74.1 & 79.4 & 92.8 & 106.4 \\
\hline 1.5 & $\mathrm{R}$ & 21.5 & 17.9 & 17.5 & 15.4 & 15.1 & 13.6 & 13.4 & 13.1 \\
\hline 4 & $\mathrm{~S}$ & 39.1 & 47.8 & 55.5 & 71.0 & 87.5 & 93.1 & 108.8 & 125.1 \\
\hline 2 & $\mathrm{R}$ & 24.5 & 20.3 & 17.6 & 17.2 & 16.9 & 15.2 & 14.8 & 14.5 \\
\hline 5 & $S$ & 37.3 & 55.6 & 64.3 & 82.2 & 89.5 & 107.3 & 125.3 & 144.2 \\
\hline 2.5 & $\mathrm{R}$ & 23.2 & 22.7 & 19.6 & 19.1 & 17.0 & 16.6 & 16.2 & 15.8 \\
\hline 6 & $S$ & 42.4 & 63.2 & 72.9 & 93.2 & 101.1 & 121.1 & 141.5 & 146.6 \\
\hline 3 & $\mathrm{R}$ & 26.3 & 25.6 & 21.9 & $21 . .4$ & 19.0 & 18.5 & 18.0 & 16.4 \\
\hline 8 & $S$ & 51.7 & 65.6 & 88.7 & 99.3 & 109.7 & 131.4 & 153.5 & 160.9 \\
\hline 4 & $\mathrm{R}$ & 31.0 & 26.2 & 25.5 & 22.4 & 20.1 & 19.6 & 19.1 & 17.5 \\
\hline 10 & $S$ & 61.0 & 77.5 & 91.6 & 117.2 & 129.0 & 154.6 & 163.9 & 188.8 \\
\hline 6 & $\mathrm{R}$ & 40.8 & 34.1 & 29.6 & 28.7 & 25.6 & 24.9 & 22.6 & 22.0 \\
\hline 12 & $S$ & 70.1 & 88.9 & 104.7 & 119.3 & 147.1 & 159.5 & 186.4 & 196.6 \\
\hline 6 & R & 40.4 & 33.7 & 29.2 & 25.9 & 25.1 & 22.8 & 22.1 & 20.1 \\
\hline 14 & $S$ & 75.1 & 95.5 & 112.6 & 128.0 & 157.9 & 170.8 & 182.7 & 194.7 \\
\hline 8 & $\mathrm{R}$ & 49.3 & 40.9 & 35.3 & 31.0 & .30 .0 & 26.9 & 24.5 & 22.5 \\
\hline 16 & $S$ & 84.0 & 106.6 & 110.9 & 141.9 & 157.9 & 172.9 & 202.0 & 215.1 \\
\hline 8 & $\mathrm{R}$ & 48.9 & 40.5 & 31.4 & 30.4 & 27.1 & 24.6 & 23.8 & 21.8 \\
\hline 18 & $S$ & 77.2 & 100.9 & 121.2 & 155.3 & 172.7 & 189.0 & 220.8 & 235.0 \\
\hline 10 & $\mathrm{R}$ & 49.2 & 41.8 & 40.2 & 35.1 & 33.7 & 30.0 & 27.2 & 26.1 \\
\hline
\end{tabular}


TABLE $B-4 . \mathrm{b}$

Economic Insulation Thickness (inch) for Steam Supply and Return Pipes for Energy Cost of $\$ 6 / \mathrm{MBtu}$

$\begin{array}{ccccccccc}\begin{array}{c}\text { Pipe } \\ \begin{array}{c}\text { Size } \\ \text { (inch) }\end{array}\end{array} & \begin{array}{l}150 \\$\cline { 2 - 7 }\end{array} & $\underline{200} & \underline{250} & \underline{300} & \underline{350} & \underline{400} & \underline{450} & \underline{500} \\ 2 \& 1 & 1.5 & 1.5 & 2.0 & 2.0 & 2.5 & 2.5 & 2.5 & 3.0 \\ 3 \& 1.5 & 1.5 & 2.0 & 2.0 & 2.5 & 2.5 & 3.0 & 3.0 & 3.0 \\ 4 \& 2 & 1.5 & 2.0 & 2.5 & 2.5 & 2.5 & 3.0 & 3.0 & 3.0 \\ 5 \& 2.5 & 2.0 & 2.0 & 2.5 & 2.5 & 3.0 & 3.0 & 3.0 & 3.0 \\ 6 \& 3 & 2.0 & 2.0 & 2.5 & 2.5 & 3.0 & 3.0 & 3.0 & 3.5 \\ 8 \& 4 & 2.0 & 2.5 & 2.5 & 3.0 & 3.5 & 3.5 & 3.5 & 4.0 \\ 10 \& 6 & 2.0 & 2.5 & 3.0 & 3.0 & 3.5 & 3.5 & 4.0 & 4.0 \\ 12 \& 6 & 2.0 & 2.5 & 3.0 & 3.5 & 3.5 & 4.0 & 4.0 & 4.5 \\ 14 \& 8 & 2.0 & 2.5 & 3.0 & 3.5 & 3.5 & 4.0 & 4.5 & 5.0 \\ 16 \& 8 & 2.0 & 2.5 & 3.5 & 3.5 & 4.0 & 4.5 & 4.5 & 5.0 \\ 18 \& 10 & 2.5 & 3.0 & 3.5 & 3.5 & 4.0 & 4.5 & 4.5 & 5.0\end{array}$

Note: Energy Cost $=\$ 6.00 / \mathrm{MBtu}$

Return Pipe Temperature $=150 \mathrm{~F}$ 
TABLE B-5.a

Maximum Al lowable Heat Losses (Btu/h.ft) from the Supply

Pipe (S) and the Return Pipe (R) for Energy Cost of $\$ 7 / M B t u$

\begin{tabular}{|c|c|c|c|c|c|c|c|c|c|}
\hline \multirow{2}{*}{$\begin{array}{l}\text { Pipe } \\
\text { Size } \\
\text { (inch) }\end{array}$} & \multirow[b]{2}{*}{$\begin{array}{l}\text { Pipe } \\
\text { Type }\end{array}$} & \multicolumn{8}{|c|}{ Process Fluid Temperature (F) } \\
\hline & & 150 & $\underline{200}$ & $\underline{250}$ & 300 & 350 & 400 & $\underline{450}$ & 500 \\
\hline 2 & S & 25.3 & 31.5 & 42.4 & 47.4 & 58.5 & 70.1 & 74.0 & 85.0 \\
\hline 1 & $\mathbf{R}$ & 17.4 & 14.8 & $14 \cdot 5$ & 12.9 & 12.7 & 12.5 & 11.4 & 11.2 \\
\hline 3 & S & 32.8 & 40.3 & 47.1 & 60.1 & 66.3 & 79.4 & 92.8 & 106.6 \\
\hline 1.5 & R & 21.5 & 17.9 & 15.7 & 15.4 & $13 \cdot 9$ & 13.6 & 13.4 & 13.1 \\
\hline 4 & $S$ & 39.1 & 47.8 & 55.5 & 71.0 & 77.7 & 93.1 & 108.8 & 113.5 \\
\hline 2 & R & 24.5 & 20.3 & 17.6 & 17.2 & 15.5 & 15.2 & 14.8 & 13.6 \\
\hline 5 & $S$ & 37.3 & 47.6 & 64.3 & 72.6 & 89.5 & 107.3 & 125.3 & 130.2 \\
\hline 2.5 & $\mathrm{R}$ & 23.2 & 20.0 & 19.6 & 17.4 & 17.0 & 16.6 & 16.2 & 14.9 \\
\hline 6 & $S$ & 42.4 & 54.0 & 72.9 & 82.0 & 101.1 & 121.1 & 127.4 & 146.6 \\
\hline 3 & $\mathrm{R}$ & 26.3 & 22.5 & 21.9 & 19.4 & 19.0 & 18.5 & 16.8 & 16.4 \\
\hline 8 & $\mathrm{~S}$ & 44.0 & 65.6 & 88.7 & 99.3 & 109.7 & 131.4 & 139.9 & 160.9 \\
\hline 4 & $\mathrm{R}$ & 26.9 & 26.2 & 25.5 & 22.4 & 20.1 & 19.6 & 18.0 & 17.5 \\
\hline 10 & $S$ & 51.8 & 67.7 & 91.6 & 104.6 & 129.0 & 140.3 & 163.9 & 173.6 \\
\hline 6 & $\mathrm{R}$ & 35.1 & 30.4 & 29.6 & 26.3 & 25.6 & 23.2 & 22.6 & 20.8 \\
\hline 12 & $S$ & 59.4 & 77.5 & 104.7 & 119.3 & 133.2 & 146.1 & 170.7 & 182.0 \\
\hline 6 & $\mathrm{R}$ & 34.8 & 30.1 & 29.2 & 25.9 & 23.4 & 21.4 & 20.8 & 19.1 \\
\hline 14 & $S$ & 63.7 & 83.2 & 100.0 & 128.0 & 142.6 & 156.4 & 169.0 & 181.6 \\
\hline 8 & $\mathrm{R}$ & 42.4 & 36.4 & 32.0 & 31.0 & 27.8 & 25.2 & 23.1 & 21.4 \\
\hline 16 & $S$ & 71.1 & 92.4 & 110.9 & 128.0 & 144.3 & 172.9 & 186.6 & 200.3 \\
\hline 8 & $\mathrm{R}$ & 42.0 & 35.9 & 31.4 & 28.1 & 25.4 & 24.6 & 22.6 & 20.9 \\
\hline 18 & $S$ & 67.2 & 89.5 & 121.2 & 140.0 & 157.7 & 189.0 & 203.8 & 218.7 \\
\hline 10 & $\mathrm{R}$ & 43.3 & 37.8 & 36.5 & 32.4 & 29.2 & 28.2 & 25.8 & 23.8 \\
\hline
\end{tabular}


TABLE $B-5 . b$

Economic Insulation Thickness (inch) for Steam Supply and Return Pipes for Energy Cost of $\$ 7 / M B t u$

\begin{tabular}{|c|c|c|c|c|c|c|c|c|}
\hline \multirow{2}{*}{$\begin{array}{l}\text { Pipe } \\
\text { Size } \\
\text { (inch) }\end{array}$} & \multicolumn{8}{|c|}{ Process Fluid Temperature (F) } \\
\hline & $\underline{150}$ & $\underline{200}$ & $\underline{250}$ & $\underline{300}$ & $\underline{350}$ & $\underline{400}$ & $\underline{450}$ & $\underline{500}$ \\
\hline $2 \& 1$ & 1.5 & 2.0 & 2.0 & 2.5 & 2.5 & 2.5 & 3.0 & 3.0 \\
\hline $3 \& 1.5$ & 1.5 & 2.0 & 2.5 & 2.5 & 3.0 & 3.0 & 3.0 & 3.0 \\
\hline $4 \& 2$ & 1.5 & 2.0 & 2.5 & 2.5 & 3.0 & 3.0 & 3.0 & 3.5 \\
\hline $5 \& 2.5$ & 2.0 & 2.5 & 2.5 & 3.0 & 3.0 & 3.0 & 3.0 & 3.5 \\
\hline $6 \& 3$ & 2.0 & 2.5 & 2.5 & 3.0 & 3.0 & 3.0 & 3.5 & 3.5 \\
\hline $8 \& 4$ & 2.5 & 2.5 & 2.5 & 3.0 & 3.5 & 3.5 & 4.0 & 4.0 \\
\hline $10 \& 6$ & 2.5 & 3.0 & 3.0 & 3.5 & 3.5 & 4.0 & 4.0 & 4.5 \\
\hline $12 \& 6$ & 2.5 & 3.0 & 3.0 & 3.5 & 4.0 & 4.5 & 4.5 & 5.0 \\
\hline $14 \& 8$ & 2.5 & 3.0 & 3.5 & 3.5 & 4.0 & 4.5 & 5.0 & 5.5 \\
\hline $16 \& 8$ & 2.5 & 3.0 & 3.5 & 4.0 & 4.5 & 4.5 & 5.0 & 5.5 \\
\hline $18 \& 10$ & 3.0 & 3.5 & 3.5 & 4.0 & 4.5 & 4.5 & 5.0 & 5.5 \\
\hline
\end{tabular}

Note: Energy Cost $=\$ 7.00 / \mathrm{MBtu}$

Return Pipe Temperature $=150 \mathrm{~F}$ 
TABLE $B-6 \cdot a$

Maximum Allowable Heat Losses (Btu/h'ft) from the Supply

Pipe (S) and the Return Pipe (R) for Energy Cost of $\$ 8 / \mathrm{MB} t u$

\begin{tabular}{|c|c|c|c|c|c|c|c|c|c|}
\hline \multirow{3}{*}{$\begin{array}{c}\text { Pipe } \\
\text { Size } \\
\text { (inch) }\end{array}$} & \multirow[b]{2}{*}{ Pipe } & \multicolumn{8}{|c|}{ Process Fluid Temperature (F) } \\
\hline & & & & & & & & & \\
\hline & Type & $\underline{150}$ & $\underline{200}$ & 250 & 300 & 350 & $\underline{400}$ & $\underline{450}$ & $\underline{500}$ \\
\hline 2 & $S$ & 25.3 & 31.5 & 37.2 & 47.4 & 58.5 & 63.3 & 74.0 & 85.0 \\
\hline 1 & $\mathrm{R}$ & 17.4 & 14.8 & 13.1 & $12 \cdot 9$ & 12.7 & 11.6 & 11.4 & 11.2 \\
\hline 3 & S & 27.1 & 40.3 & 47.1 & 60.1 & 66.3 & 79.4 & 92.8 & 106.6 \\
\hline 1.5 & $\mathbf{R}$ & 18.2 & 17.9 & 15.7 & 15.4 & 13.9 & 13.6 & 13.4 & 13.1 \\
\hline 4 & $S$ & 32.1 & 41.2 & 55.5 & 71.0 & 77.7 & 93.1 & 98.7 & 113.5 \\
\hline 2 & $\mathrm{R}$ & 20.7 & 18.0 & 17.6 & 17.2 & 15.5 & 15.2 & 13.9 & 13.6 \\
\hline 5 & $S$ & 37.3 & 47.6 & 64.3 & 72.6 & 89.5 & 107.3 & 113.2 & 130.2 \\
\hline 2.5 & $\mathrm{R}$ & 23.2 & 20.0 & 19.6 & 17.4 & 17.0 & 16.6 & 15.2 & 14.9 \\
\hline 6 & S & 42.4 & 54.0 & 64.2 & 82.0 & 101.1 & 109.0 & 127.4 & 134.1 \\
\hline 3 & $\mathrm{R}$. & 26.3 & 22.5 & 19.9 & 19.4 & 19.0 & 17.2 & 16.8 & 15.5 \\
\hline 8 & $S$ & 44.0 & 65.6 & 77.7 & 89.0 & 109.7 & 119.7 & 139.9 & 148.4 \\
\hline 4 & $\mathrm{R}$ & 26.9 & 26.2 & 23.0 & 20.6 & 20.1 & 18.4 & 18.0 & 16.7 \\
\hline 10 & $S$ & 51.8 & 67.7 & 81.8 & 104.6 & 117.1 & 140.3 & 150.8 & 173.6 \\
\hline 6 & $\mathrm{R}$ & 35.1 & 30.4 & 27.0 & 26.3 & 23.8 & 23.2 & 21.4 & 20.8 \\
\hline 12 & $S$ & 59.4 & 77.5 & 93.3 & 108.0 & 122.0 & 146.1 & 158.1 & 182.0 \\
\hline 6 & $\mathrm{R}$ & 34.8 & 30.1 & 26.7 & 24.1 & 21.9 & 21.4 & 19.7 & 19.1 \\
\hline 14 & $S$ & 63.7 & 83.2 & 100.0 & 115.6 & 130.5 & 156.4 & 169.0 & 181.6 \\
\hline 8 & $\mathrm{R}$ & 42.4 & 36.4 & 32.0 & 28.6 & 25.9 & 25.2 & 23.1 & 21.4 \\
\hline 16 & $S$ & 61.7 & 82.0 & 110.1 & 117.1 & 144.3 & 172.9 & 186.6 & 200.3 \\
\hline 8 & $\mathrm{R}$ & 37.0 & 32.4 & 28.9 & 26.2 & 25.4 & 24.6 & 22.6 & 20.9 \\
\hline 18 & $S$ & 67.2 & 89.5 & 109.4 & 127.9 & 157.7 & 174.5 & 189.7 & 218.7 \\
\hline 10 & $\mathrm{R}$ & 43.3 & 37.8 & 33.5 & 30.2 & 29.2 & 26.7 & 24.6 & 23.8 \\
\hline
\end{tabular}

Note: Energy Cost $=\$ 8.00 / \mathrm{MEti}$

Return Pipe Temperature $=150 \mathrm{~F}$ 
TABLE $B-6 . b$

Economic Insulation Thickness (inch) for Steam Supply and Return Pipes for Energy Cost of $\$ 8 / \mathrm{MBtu}$

\begin{tabular}{|c|c|c|c|c|c|c|c|c|}
\hline \multirow{2}{*}{$\begin{array}{c}\text { Pipe } \\
\text { Size } \\
\text { (inch) }\end{array}$} & \multicolumn{8}{|c|}{ Process Fluid Temperature (F) } \\
\hline & 150 & $\underline{200}$ & $\underline{250}$ & $\underline{300}$ & 350 & 400 & 450 & $\underline{500}$ \\
\hline $2 \& 1$ & 1.5 & 2.0 & 2.5 & 2.5 & 2.5 & 3.0 & 3.0 & 3.0 \\
\hline $3 \& 1.5$ & 2.0 & 2.0 & 2.5 & 2.5 & 3.0 & 3.0 & 3.0 & 3.0 \\
\hline $4 \& 2$ & 2.0 & 2.5 & 2.5 & 2.5 & 3.0 & 3.0 & 3.5 & 3.5 \\
\hline $5 \& 2.5$ & 2.0 & 2.5 & 2.5 & 3.0 & 3.0 & 3.0 & 3.5 & 3.5 \\
\hline $6 \& 3$ & 2.0 & 2.5 & 3.0 & 3.0 & 3.0 & 3.5 & 3.5 & 4.0 \\
\hline $8 \& 4$ & 2.5 & 2.5 & 3.0 & 3.5 & 3.5 & 4.0 & 4.0 & 4.5 \\
\hline $10 \& 6$ & 2.5 & 3.0 & 3.5 & 3.5 & 4.0 & 4.0 & 4.5 & 4.5 \\
\hline $12 \& 6$ & 2.5 & 3.0 & 3.5 & $4.0^{\circ}$ & 4.5 & 4.5 & 5.0 & 5.0 \\
\hline $14 \& 8$ & 2.5 & 3.0 & 3.5 & 4.0 & 4.5 & 4.5 & 5.0 & 5.5 \\
\hline $16 \& 8$ & 3.0 & 3.5 & 4.0 & 4.5 & 4.5 & 4.5 & 5.0 & 5.5 \\
\hline $18 \& 10$ & 3.0 & 3.5 & 4.0 & 4.5 & 4.5 & 5.0 & 5.5 & 5.5 \\
\hline
\end{tabular}

Note: Energy Cost $=\$ 8.00 / \mathrm{MBtu}$

Return Pipe Temperature $=150 \mathrm{~F}$ 
TABLE B-7.a

Maximum Allowable Heat Losses (Btu/h.ft) from the Supply Pipe (S) and the Return Pipe (R) for Energy Cost of $\$ 9 / M B t u$

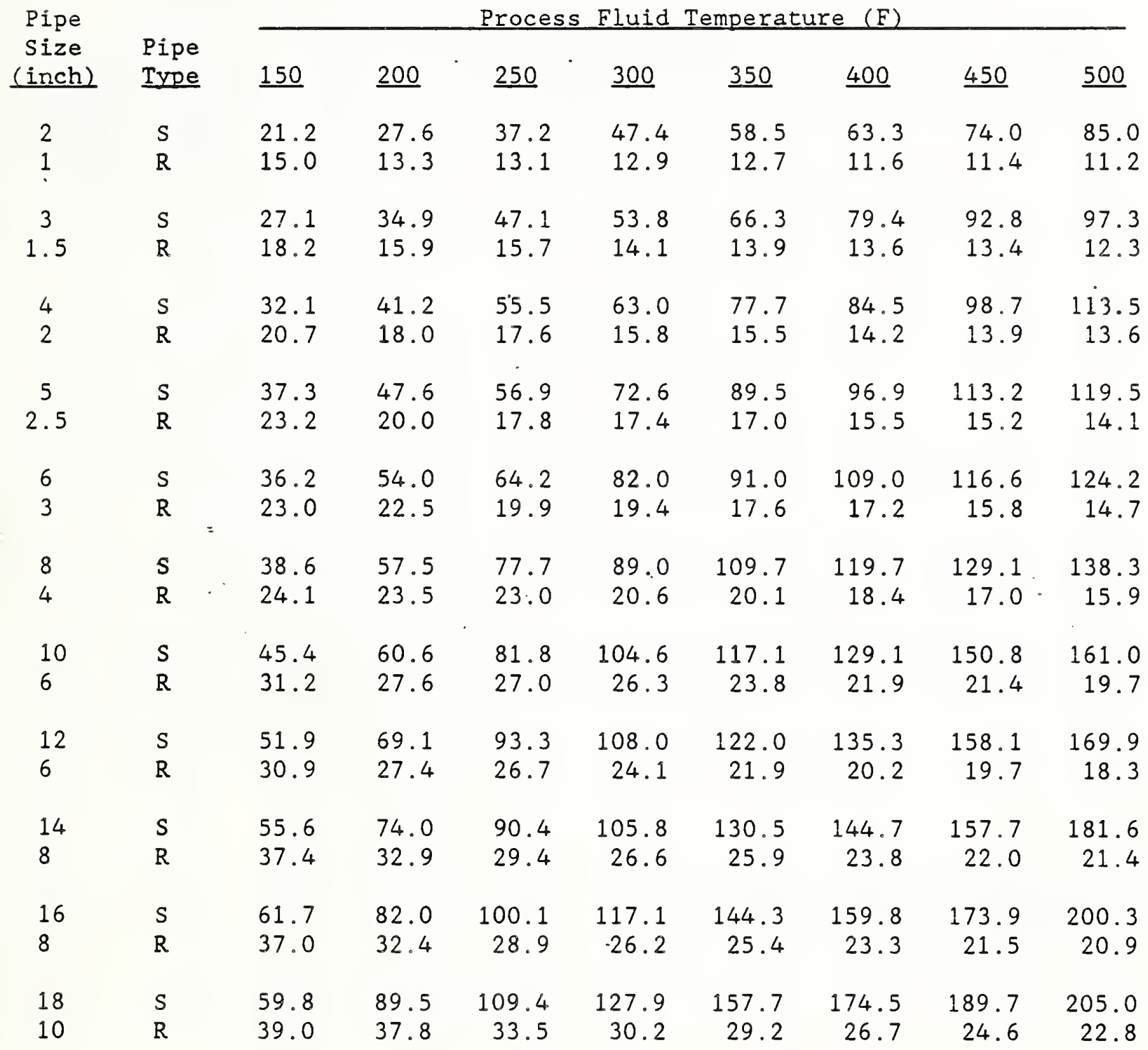

Note: Energy Cost $=\$ 9.00 / \mathrm{MBtu}$

Return Pipe Temperature $=150 \mathrm{~F}$ 
TABLE $B-7 . b$

Economic Insulation Thickness (inch) for Steam Supply and Return Pipes for Energy Cost of $\$ 9 / \mathrm{MBtu}$

\begin{tabular}{|c|c|c|c|c|c|c|c|c|}
\hline \multirow{2}{*}{$\begin{array}{c}\text { Pipe } \\
\text { Size } \\
\text { (inch) } \\
\end{array}$} & \multicolumn{8}{|c|}{ Process Fluid Temperature (F) } \\
\hline & $\underline{150}$ & $\underline{200}$ & $\underline{250}$ & $\underline{300}$ & $\underline{350}$ & $\underline{400}$ & 450 & 500 \\
\hline $2 \& 1$ & 2.0 & 2.5 & 2.5 & 2.5 & 2.5 & 3.0 & 3.0 & 3.0 \\
\hline $3 \& 1.5$ & 2.0 & 2.5 & 2.5 & 3.0 & 3.0 & 3.0 & 3.0 & 3.5 \\
\hline $4 \& 2$ & 2.0 & 2.5 & 2.5 & 3.0 & 3.0 & 3.5 & 3.5 & 3.5 \\
\hline $5 \& 2.5$ & 2.0 & 2.5 & 3.0 & 3.0 & 3.0 & 3.5 & 3.5 & 4.0 \\
\hline $6 \& 3$ & 2.5 & 2.5 & 3.0 & 3.0 & 3.5 & 3.5 & 4.0 & 4.5 \\
\hline $8 \& 4$ & 3.0 & 3.0 & 3.0 & 3.5 & 3.5 & 4.0 & 4.5 & 5.0 \\
\hline $10 \& 6$ & 3.0 & 3.5 & 3.5 & 3.5 & 4.0 & 4.5 & 4.5 & 5.0 \\
\hline $12 \& 6$ & 3.0 & 3.5 & 3.5 & 4.0 & 4.5 & 5.0 & 5.0 & 5.5 \\
\hline $14 \& 8$ & 3.0 & 3.5 & 4.0 & 4.5 & 4.5 & 5.0 & 5.5 & 5.5 \\
\hline $16 \& 8$ & 3.0 & 3.5 & 4.0 & 4.5 & 4.5 & 5.0 & 5.5 & 5.5 \\
\hline $18 \& 10$ & 3.5 & 3.5 & 4.0 & 4.5 & 4.5 & 5.0 & 5.5 & 6.0 \\
\hline
\end{tabular}

Note: Energy Cost $=\$ 9.00 / \mathrm{MBtu}$

Return Pipe Temperature $=150 \mathrm{~F}$ 
TABLE $B-8 . a$

Maximum Allowable Heat Losses (Btu/h $\mathrm{ft}$ ) from the Supply

Pipe (S) and the Return Pipe (R) for Energy Cost of $\$ 10 / M B t u$

\begin{tabular}{|c|c|c|c|c|c|c|c|c|c|}
\hline \multirow{3}{*}{$\begin{array}{l}\text { Pipe } \\
\text { Size } \\
\text { (inch) }\end{array}$} & \multirow[b]{2}{*}{ Pipe } & \multicolumn{8}{|c|}{ Process Fluid Temperature (F) } \\
\hline & & & & & & & & & \\
\hline & Type & $\underline{150}$ & $\underline{200}$ & $\underline{250}$ & 300 & 350 & 400 & 450 & $\underline{500}$ \\
\hline 2 & S & 21.2 & 27.6 & 37.2 & 47.4 & 52.8 & 63.3 & 74.0 & 78.2 \\
\hline 1 & $\mathrm{R}$ & 15.0 & 13.3 & 13.1 & 12.9 & 11.7 & 11.6 & 11.4 & 10.6 \\
\hline 3 & s & 27.1 & 34.9 & 47.1 & 53.8 & 66.3 & 79.4 & 84.7 & 97.3 \\
\hline 1.5 & $\mathbf{R}$ & 18.2 & 15.9 & 15.7 & 14.1 & 13.9 & 13.6 & 12.6 & 12.3 \\
\hline 4 & S & 27.7 & 41.2 & 55.5 & 63.0 & 77.7 & 84.5 & 98.7 & 113.5 \\
\hline 2 & $\mathrm{R}$ & 18.3 & 18.0 & 17.6 & 15.8 & 15.5 & 14.2 & 13.9 & 13.6 \\
\hline 5 & S & 32.0 & 47.6 & 56.9 & 72.6 & 89.5 & 96.9 & 113.2 & 119.5 \\
\hline 2.5 & $\mathrm{R}$ & 20.4 & 20.0 & 17.8 & 17.4 & 17.0 & 15.5 & 15.2 & 14.1 \\
\hline 6 & S & 36.2 & 54.0 & 64.2 & 82.0 & 91.0 & 109.0 & 116.6 & 124.2 \\
\hline 3 & $\mathbf{R}$ & 23.0 & 22.5 & 19.9 & 19.4 & 17.6 & 17.2 & 15.8 & 14.7 \\
\hline 8 & $S$ & 38.6 & 57.5 & 69.6 & 89.0 & 99.9 & 110.5 & 129.1 & 138.3 \\
\hline 4 & $\mathrm{R}$ & 24.1 & 23.5 & 21.1 & 20.6 & 18.8 & 17.4 & 17.0 & 15.9 \\
\hline 10 & $S$ & 45.4 & 60.6 & 81.8 & 95.0 & 107.8 & 129.1 & 140.0 & 150.6 \\
\hline 6 & $R$ & 31.2 & 27.6 & 27.0 & 24.4 & 22.4 & 21.9 & 20.2 & 18.8 \\
\hline 12 & $S$ & 51.9 & 69.1 & 84.5 & 98.9 & 112.9 & 135.3 & 147.6 & 159.7 \\
\hline 6 & $\mathrm{R}$ & 30.9 & 27.4 & 24.7 & 22.5 & 20.7 & 20.2 & 18.8 & 17.5 \\
\hline 14 & $S$ & 55.6 & 74.0 & 90.4 & 105.8 & 120.7 & 135.0 & 157.7 & 170.5 \\
\hline 8 & $\mathrm{R}$ & 37.4 & 32.9 & 29.4 & 26.6 & 24.4 & 22.6 & 22.0 & 20.5 \\
\hline 16 & $S$ & 54.9 & 74.0 & 91.5 & 117.1 & 133.4 & 148.9 & 173.9 & 187.9 \\
\hline 8 & $\mathbf{R}$ & 33.4 & 29.8 & 26.9 & 26.2 & 24.0 & 22.1 & 21.5 & 20.0 \\
\hline 18 & S & 59.8 & 80.8 & 99.9 & 127.9 & 145.6 & 162.4 & 177.9 & 205.0 \\
\hline 10 & $\mathbf{R}$ & 39.0 & 34.6 & 31.2 & 30.2 & 27.2 & $25.4^{\circ}$ & 23.5 & 22.8 \\
\hline
\end{tabular}


TABLE $B-8 \cdot b$

Economic Insulation Thickness (inch) for Steam Supply
and Return Pipes for Energy Cost of $\$ 10 / \mathrm{MBtu}$

\begin{tabular}{|c|c|c|c|c|c|c|c|c|}
\hline \multirow{3}{*}{$\begin{array}{l}\text { Pipe } \\
\text { Size } \\
\text { (inch) }\end{array}$} & \multicolumn{8}{|c|}{ Process Fluid Temperature (F) } \\
\hline & & & & & & & & \\
\hline & $\underline{150}$ & $\underline{200}$ & $\underline{250}$ & $\underline{300}$ & $\underline{350}$ & $\underline{400}$ & $\underline{450}$ & $\underline{500}$ \\
\hline $2 \& 1$ & 2.0 & 2.5 & 2.5 & 2.5 & 3.0 & 3.0 & 3.0 & 3.5 \\
\hline $3 \&, 1.5$ & 2.0 & 2.5 & 2.5 & 3.0 & 3.0 & 3.0 & 3.5 & 3.5 \\
\hline $4 \& 2$ & 2.5 & 2.5 & 2.5 & 3.0 & 3.0 & 3.5 & 3.5 & 3.5 \\
\hline $5 \& 2.5$ & 2.5 & 2.5 & 3.0 & 3.0 & 3.0 & 3.5 & 3.5 & 4.0 \\
\hline $6 \& 3$ & 2.5 & 2.5 & 3.0 & 3.0 & 3.5 & 3.5 & 4.0 & 4.5 \\
\hline $8 \& 4$ & 3.0 & 3.0 & 3.5 & 3.5 & 4.0 & 4.5 & 4.5 & 5.0 \\
\hline $10 \& 6$ & 3.0 & 3.5 & 3.5 & 4.0 & 4.5 & 4.5 & 5.0 & 5.5 \\
\hline $12 \& 6$ & 3.0 & 3.5 & 4.0 & 4.5 & 5.0 & 5.0 & 5.5 & 6.0 \\
\hline $14 \& 8$ & 3.0 & 3.5 & .4 .0 & 4.5 & 5.0 & 5.5 & 5.5 & 6.0 \\
\hline $16 \& 8$ & 3.5 & 4.0 & 4.5 & 4.5 & 5.0 & 5.5 & 5.5 & 6.0 \\
\hline $18 \& 10$ & 3.5 & 4.0 & 4.5 & 4.5 & 5.0 & 5.5 & 6.0 & 6.0 \\
\hline
\end{tabular}

Note: Energy Cost $=\$ 10.00 / \mathrm{MBtu}$

Return Pipe Temperature $=150 \mathrm{~F}$ 
TABLE B-9.a

Maximum Allowable Heat Losses $(B t u / h \cdot f t)$ from the Supply

Pipe (S) and the Return Pipe (R) for Energy Cost of $\$ 11 / M B t u$

\begin{tabular}{|c|c|c|c|c|c|c|c|c|c|}
\hline \multirow{3}{*}{$\begin{array}{l}\text { Pipe } \\
\text { Size } \\
\text { (inch) }\end{array}$} & \multirow[b]{2}{*}{ Pipe } & \multicolumn{8}{|c|}{ Process Fluid Temperature (F) } \\
\hline & & 150 & 200 & 250 & 300 & 350 & 400 & 450 & 500 \\
\hline & & 120 & $\underline{200}$ & $\underline{250}$ & 300 & 320 & $\underline{400}$ & 450 & $\underline{500}$ \\
\hline 2 & $S$ & 18.6 & 27.6 & 37.2 & 42.8 & 52.8 & 63.3 & 68.1 & 78.2 \\
\hline 1 & $\mathrm{R}$ & $13 \cdot 4$ & 13.3 & 13.1 & $11 \cdot 9$ & 11.7 & 11.6 & 10.7 & 10.6 \\
\hline 3 & S & 23.5 & 34.9 & 42.1 & 53.8 & 66.3 & 72.5 & 84.7 & 97.3 \\
\hline 1.5 & $\mathbf{R}$ & 18.2 & 15.9 & 14.4 & 14.1 & 13.9 & 12.8 & 12.6 & 12.3 \\
\hline 4 & $S$ & 27.7 & 41.2 & 49.4 & 63.0 & 70.6 & 84.5 & 98.7 & 113.5 \\
\hline 2 & $\mathrm{R}$ & 18.3 & 18.0 & 16.1 & 15.8 & 14.4 & 14.2 & 13.9 & 13.6 \\
\hline 5 & $S$ & 32.0 & 42.2 & 56.9 & 72.6 & 80.9 & 96.9 & 104.0 & 119.5 \\
\hline 2.5 & $\mathrm{R}$ & 20.4 & 18.1 & 17.8 & 17.4 & 15.8 & 15.5 & 14.4 & 14.1 \\
\hline 6 & $S$ & 36.2 & 47.6 & 64.2 & 73.8 & 91.0 & 99.8 & 108.1 & 116.2 \\
\hline 3 & R & 23.0 & 20.3 & 19.9 & 17.9 & 17.6 & 16.2 & 15.0 & 14.1 \\
\hline 8 & $S$ & 38.6 & 57.5 & 69.6 & 89.0 & 99.9 & 110.5 & 120.3 & 129.9 \\
\hline 4 & $\mathrm{R}$ & 24.1 & 23.5 & 21.1 & 20.6 & 18.8 & 17.4 & 16.2 & 15.3 \\
\hline 10 & $S$ & 45.4 & 60.6 & 74.3 & 95.0 & 107.8 & 119.8 & 140.0 & 150.6 \\
\hline 6 & $\mathrm{R}$ & 31.2 & 27.6 & 25.0 & 24.4 & 22.4 & 20.6 & 20.2 & 18.8 \\
\hline 12 & $S$ & 46.4 & 69.1 & 84.5 & 98.9 & 112.9 & 126.3 & 147.6 & 159.7 \\
\hline 6 & R & 28.0 & 27.4 & 24.7 & 22.5 & 20.7 & 19.2 & 18.8 & 17.5 \\
\hline 14 & S & 49.5 & 74.0 & 90.4 & 105.8 & 120.7 & 135.0 & 157.7 & 170.5 \\
\hline 8 & $\mathrm{R}$ & 33.7 & 32.9 & 29.4 & 26.6 & 24.4 & 22.6 & 22.0 & 20.5 \\
\hline 16 & S & 54.9 & 74.0 & 91.5 & 117.1 & 133.4 & 148.9 & 163.2 & 187.9 \\
\hline 8 & $\mathrm{R}$ & 33.4 & 29.8 & 26.9 & 26.2 & 24.0 & 22.1 & 20.6 & 20.0 \\
\hline 18 & $S$ & 59.8 & 80.8 & 99.9 & 118.1 & 135.6 & 162.4 & 177.9 & 205.0 \\
\hline 10 & $\mathrm{R}$ & 39.0 & 34.6 & 31.2 & 28.4 & 26.1 & 25.4 & 23.5 & 22.8 \\
\hline
\end{tabular}


TABLE $B-9 \cdot b$

Economic Insulation Thickness (inch) for Steam Supply and Return Pipes for Energy Cost of $\$ 11 /$ MBtu

\begin{tabular}{|c|c|c|c|c|c|c|c|c|}
\hline \multirow{2}{*}{$\begin{array}{l}\text { Pipe } \\
\text { Size } \\
\text { (inch) } \\
\end{array}$} & \multicolumn{8}{|c|}{ Process Fluid Temperature (F) } \\
\hline & $\underline{150}$ & $\underline{200}$ & $\underline{250}$ & 300 & $\underline{350}$ & 400 & 450 & $\underline{500}$ \\
\hline $2 \& 1$ & 2.5 & 2.5 & 2.5 & 3.0 & 3.0 & 3.0 & 3.5 & 3.5 \\
\hline $3 \& 1.5$ & 2.5 & 2.5 & 3.0 & 3.0 & 3.0 & 3.5 & 3.5 & 3.5 \\
\hline $4 \& 2$ & 2.5 & 2.5 & 3.0 & 3.0 & 3.5 & 3.5 & 3.5 & 3.5 \\
\hline $5 \& 2.5$ & 2.5 & 3.0 & 3.0 & 3.0 & 3.5 & 3.5 & 4.0 & 4.0 \\
\hline $6 \& 3$ & 2.5 & 3.0 & 3.0 & 3.5 & 3.5 & 4.0 & 4.5 & 5.0 \\
\hline $8 \& 4$ & 3.0 & 3.0 & 3.5 & 3.5 & 4.0 & 4.5 & 5.0 & 5.5 \\
\hline $10 \& 6$ & 3.0 & 3.5 & 4.0 & 4.0 & 4.5 & 5.0 & 5.0 & 5.5 \\
\hline $12 \& 6$ & -3.5 & 3.5 & 4.0 & 4.5 & 5.0 & 5.5 & 5.5 & 6.0 \\
\hline $14 \& 8$ & 3.5 & 3.5 & 4.0 & 4.5 & 5.0 & 5.5 & 5.5 & 6.0 \\
\hline $16 \& 8$ & 3.5 & 4.0 & 4.5 & 4.5 & 5.0 & 5.5 & 6.0 & 6.0 \\
\hline $18 \& 10$ & 3.5 & 4.0 & 4.5 & 5.0 & 5.5 & 5.5 & 6.0 & 6.0 \\
\hline
\end{tabular}

Note: Energy Cost $=\$ 11.00 / \mathrm{MBtu}$

Return Pipe Temperature $=150 \mathrm{~F}$ 
TABLE $B-10 . a$

Maximum Allowable Heat Losses (Btu/h•ft) from the Supply Pipe (S) and the Return Pipe (R) for Energy Cost of $\$ 12 / \mathrm{MBtu}$

\begin{tabular}{|c|c|c|c|c|c|c|c|c|c|}
\hline \multirow{3}{*}{$\begin{array}{l}\text { Pipe } \\
\text { Size } \\
\text { (inch) }\end{array}$} & \multirow[b]{2}{*}{ P ipe } & \multicolumn{8}{|c|}{ Process Fluid Temperature (F) } \\
\hline & & & & & & & & & \\
\hline & Type & $\underline{150}$ & $\underline{200}$ & $\underline{250}$ & 300 & 350 & $\underline{400}$ & 450 & $\underline{500}$ \\
\hline 2 & S & 18.6 & 27.6 & 37.2 & 42.8 & 52.8 & 63.3 & 68.1 & 78.2 \\
\hline 1 & $\mathbf{R}$ & 13.4 & 13.3 & 13.1 & 11.9 & 11.7 & 11.6 & 10.7 & $10 \cdot 6$ \\
\hline 3 & S & 23.5 & 34.9 & 42.1 & 53.8 & 66.3 & 72.5 & .84 .7 & 90.1 \\
\hline 1.5 & $\mathbf{R}$ & 16.2 & 15.9 & 14.4 & 14.1 & 13.9 & 12.8 & 12.6 & 11.7 \\
\hline 4 & $S$ & 27.7 & 41.2 & 49.4 & 63.0 & 70.6 & 84.5 & 98.7 & 104.6 \\
\hline 2 & $\mathbf{R}$ & 18.3 & 18.0 & 16.1 & 15.8 & 14.4 & 14.2 & 13.9 & 12.9 \\
\hline 5 & $S$ & 32.0 & 42.2 & 56.9 & 72.6 & 80.9 & 96.9 & 104.0 & 119.5 \\
\hline 2.5 & $\mathbf{R}$ & 20.4 & 18.1 & 17.8 & 17.4 & 15.8 & 15.5 & 14.4 & 14.1 \\
\hline 6 & $s=$ & 36.2 & 47.6 & 64.2 & 73.8 & 91.0 & 99.8 & 108.1 & 116.2 \\
\hline 3 & $\mathrm{R}$ & 23.0 & 20.3 & 19.9 & 17.9 & 17.6 & 16.2 & 15.0 & 14.1 \\
\hline 8 & $S$ & 34.7 & 51.6 & 69.6 & 81.1 & 92.2 & 102.9 & 120.3 & 129.9 \\
\hline 4 & $\mathbf{R}$ & 22.0 & 21.5 & 21.1 & 19.2 & 17.8 & 16.6 & 16.2 & 15.3 \\
\hline 10 & $S$ & 40.6 & 60.6 & 74.3 & 87.4 & 107.8 & 119.8 & 131.0 & 150.6 \\
\hline 6 & $\mathbf{R}$ & 28.2 & 27.6 & 25.0 & 22.8 & 22.4 & 20.6 & 19.2 & 18.8 \\
\hline 12 & $S$ & 46.4 & 62.6 & 77.4 & 91.6 & 112.9 & 126.3 & 138.8 & 159.7 \\
\hline 6 & $\mathbf{R}$ & 28.0 & 25.3 & 23.0 & 21.2 & 20.7 & 19.2 & 18.0 & 17.5 \\
\hline 14 & $S$ & 49.5 & 66.9 & 82.8 & 97.9 & 112.7 & 135.0 & 148.2 & 170.5 \\
\hline 8 & $\mathbf{R}$ & 33.7 & 30.1 & 27.3 & 25.0 & 23.1 & 22.6 & 21.0 & 20.5 \\
\hline 16 & $S$ & 54.9 & 74.0 & 91.5 & 108.1 & 124.3 & 148.9 & 163.2 & 187.9 \\
\hline 8 & $\mathbf{R}$ & 33.4 & 29.8 & 26.9 & 24.6 & .22 .7 & 22.1 & 20.6 & 20.0 \\
\hline 18 & $S$ & 59.8 & 80.8 & 99.9 & 118.1 & 135.6 & 152.3 & 177.9 & 205.0 \\
\hline 10 & $\mathbf{R}$ & 39.0 & 34.6 & 31.2 & 28.4 & 26.1 & 24.2 & 23.5 & 22.8 \\
\hline
\end{tabular}


TABLE B-10.b

Economic Insulation Thickness (inch) for Steam Supply
and Return Pipes for Energy Cost of $\$ 12 /$ MBtu

\begin{tabular}{|c|c|c|c|c|c|c|c|c|}
\hline \multirow{2}{*}{$\begin{array}{c}\text { Pipe } \\
\text { Size } \\
\text { (inch) }\end{array}$} & \multicolumn{8}{|c|}{ Process Fluid Temperature (F) } \\
\hline & $\underline{150}$ & $\underline{200}$ & $\underline{250}$ & $\underline{300}$ & $\underline{350}$ & $\underline{400}$ & $\underline{450}$ & $\underline{500}$ \\
\hline $2 \& 1$ & 2.5 & 2.5 & 2.5 & 3.0 & 3.0 & 3.0 & 3.5 & 3.5 \\
\hline $3 \& 1.5$ & 2.5 & 2.5 & 3.0 & 3.0 & 3.0 & 3.5 & 3.5 & 4.0 \\
\hline $4 \& 2$ & 2.5 & 2.5 & 3.0 & 3.0 & 3.5 & 3.5 & 3.5 & 4.0 \\
\hline $5 \& 2.5$ & 2.5 & 3.0 & 3.0 & 3.0 & 3.5 & 3.5 & 4.0 & 4.0 \\
\hline $6 \& 3$ & 2.5 & 3.0 & 3.0 & 3.5 & 3.5 & 4.0 & 4.5 & 5.0 \\
\hline $8 \& 4$ & 3.5 & 3.5 & 3.5 & 4.0 & 4.5 & 5.0 & 5.0 & 5.5 \\
\hline $10 \& 6$ & 3.5 & 3.5 & 4.0 & 4.5 & 4.5 & 5.0 & 5.5 & 5.5 \\
\hline $12 \& 6$ & 3.5 & 4.0 & 4.5 & 5.0 & 5.0 & 5.5 & 6.0 & 6.0 \\
\hline $14 \& 8$ & 3.5 & 4.0 & 4.5 & 5.0 & 5.5 & 5.5 & 6.0 & 6.0 \\
\hline $16 \& 8$ & 3.5 & 4.0 & 4.5 & 5.0 & 5.5 & 5.5 & 6.0 & 6.0 \\
\hline $18 \& 10$ & 3.5 & 4.0 & 4.5 & 5.0 & 5.5 & 6.0 & 6.0 & 6.0 \\
\hline
\end{tabular}

Note: Energy Cost $=\$ 12.00 / \mathrm{MBtu}$

Return Pipe Temperature $=150 \mathrm{~F}$ 
TABLE B-11.a

Maximum Allowable Heat Losses (Btu/h $\mathrm{ft}$ ) from the Supply

Pipe (S) and the Return Pipe (R) for Energy Cost of $\$ 13 /$ MBtu

\begin{tabular}{|c|c|c|c|c|c|c|c|c|c|}
\hline \multirow{2}{*}{$\begin{array}{l}\text { Pipe } \\
\text { Size } \\
\text { (inch) }\end{array}$} & \multirow[b]{2}{*}{$\begin{array}{l}\text { Pipe } \\
\text { Type }\end{array}$} & \multicolumn{8}{|c|}{ Process Fluid Temperature (F) } \\
\hline & & 150 & $\underline{200}$ & 250 & 300 & 350 & 400 & $\underline{450}$ & 500 \\
\hline 2 & $S$ & 18.6 & 27.6 & 33.6 & 42.8 & 52.8 & 58.3 & 68.1 & 78.2 \\
\hline 1 & $\mathbf{R}$ & 13.4 & $13 \cdot 3$ & 12.1 & 11.9 & 11.7 & 10.9 & 10.7 & 10.6 \\
\hline 3 & S & 23.5 & 31.3 & 42.1 & 53.8 & 60.5 & 72.5 & 84.7 & 90.1 \\
\hline 1.5 & $\mathrm{R}$ & 16.2 & 14.6 & 14.4 & 14.1 & 13.0 & 12.8 & 12.6 & 11.7 \\
\hline 4 & $S$ & 27.7 & 36.6 & 49.4 & 57.2 & 70.6 & 84.5 & 98.7 & 104.6 \\
\hline 2 & $\mathrm{R}$ & 18.3 & 16.3 & 16.1 & 14.7 & 14.4 & 14.2 & 13.9 & 12.9 \\
\hline 5 & $S$ & 32.0 & 42.2 & 56.9 & 65.6 & 80.9 & 96.9 & 104.0 & 119.5 \\
\hline 2.5 & $\mathrm{R}$ & 20.4 & 18.1 & 17.8 & 16.1 & 15.8 & 15.5 & 14.4 & 14.1 \\
\hline 6 & $s^{=}$ & 32.0 & 47.6 & 57.8 & 73.8 & 83.3 & 99.8 & 108.1 & 116.2 \\
\hline 3 & $\mathbf{R}$ & 20.7 & 20.3 & 18.3 & 17.9 & 16.5 & 16.2 & 15.0 & 14.1 \\
\hline 8 & $S$ & 34.7 & 51.6 & 69.6 & 81.1 & $92: 2$ & 102.9 & 113.0 & 129.9 \\
\hline 4 & $\mathbf{R}$ & 22.0 & 21.5 & 21.1 & 19.2 & 17.8 & 16.6 & 15.6 & 15.3 \\
\hline 10 & $S$ & 40.6 & 60.6 & 74.3 & 87.4 & 100.0 & 112.1 & 131.0 & 141.9 \\
\hline 6 & $\mathrm{R}$ & 28.2 & 27.6 & 25.0 & 22.8 & 21.1 & 19.6 & 19.2 & 18.0 \\
\hline 12 & $S$ & 46.4 & 62.6 & 77.4 & 91.6 & 105.5 & 118.8 & 138.8 & 159.7 \\
\hline 6 & $\mathbf{R}$ & 28.0 & 25.3 & 23.0 & 21.2 & 19.6 & 18.4 & 18.0 & 17.5 \\
\hline 14 & $S$ & 49.5 & 66.9 & 82.8 & 97.9 & 112.7 & 126.8 & 148.2 & 170.5 \\
\hline 8 & R & 33.7 & 30.1 & 27.3 & 25.0 & 23.1 & 21.5 & 21.0 & 20.5 \\
\hline 16 & $S$ & 49.6 & 67.7 & 91.5 & 108.1 & 124.3 & 139.7 & 163.2 & 187.9 \\
\hline 8 & $\mathbf{R}$ & 30.5 & 27.6 & 26.9 & 24.6 & 22.7 & 21.2 & 20.6 & 20.0 \\
\hline $\begin{array}{l}18 \\
10\end{array}$ & $\begin{array}{l}\mathrm{S} \\
\mathrm{R}\end{array}$ & $\begin{array}{l}54.0 \\
35.5\end{array}$ & $\begin{array}{l}73.9 \\
32.0\end{array}$ & $\begin{array}{l}92.3 \\
29.2\end{array}$ & $\begin{array}{r}109.9 \\
26.8\end{array}$ & $\begin{array}{r}127.1 \\
24.9\end{array}$ & $\begin{array}{r}152.3 \\
24.2\end{array}$ & $\begin{array}{r}177.9 \\
23.5\end{array}$ & $\begin{array}{r}205.0 \\
22.8\end{array}$ \\
\hline
\end{tabular}


TABLE B-11.b Economic Insulation Thickness (inch) for Steam Supply
and Return Pipes for Energy Cost of $\$ 13 /$ MBtu

\begin{tabular}{|c|c|c|c|c|c|c|c|c|}
\hline \multirow{2}{*}{$\begin{array}{l}\text { Pipe } \\
\text { Size } \\
\text { (inch) } \\
\end{array}$} & \multicolumn{8}{|c|}{ Process Fluid Temperature (F) } \\
\hline & $\underline{150}$ & $\underline{200}$ & 250 & $\underline{300}$ & 350 & 400 & 450 & $\underline{500}$ \\
\hline $2 \& 1$ & 2.5 & 2.5 & 3.0 & 3.0 & 3.0 & 3.5 & 3.5 & 3.5 \\
\hline $3 \& 1.5$ & 2.5 & 3.0 & 3.0 & 3.0 & 3.5 & 3.5 & 3.5 & 4.0 \\
\hline $4 \& 2$ & 2.5 & 3.0 & 3.0 & 3.5 & 3.5 & 3.5 & 3.5 & 4.0 \\
\hline $5 \& 2.5$ & 2.5 & 3.0 & 3.0 & 3.5 & 3.5 & 3.5 & 4.0 & 4.0 \\
\hline $6 \& 3$ & 3.0 & 3.0 & 3.5 & 3.5 & 4.0 & 4.0 & 4.5 & 5.0 \\
\hline $8 \& 4$ & 3.5 & 3.5 & 3.5 & 4.0 & 4.5 & 5.0 & 5.5 & 5.5 \\
\hline $10 \& 6$ & 3.5 & 3.5 & 4.0 & 4.5 & 5.0 & 5.5 & 5.5 & 6.0 \\
\hline $12 \& 6$ & 3.5 & 4.0 & 4.5 & 5.0 & 5.5 & 6.0 & 6.0 & 6.0 \\
\hline $14 \& 8$ & 3.5 & 4.0 & 4.5 & 5.0 & 5.5 & 6.0 & 6.0 & 6.0 \\
\hline $16 \& 8$ & 4.0 & 4.5 & 4.5 & 5.0 & 5.5 & 6.0 & 6.0 & 6.0 \\
\hline $18 \& 10$ & 4.0 & 4.5 & 5.0 & 5.5 & 6.0 & 6.0 & 6.0 & 6.0 \\
\hline
\end{tabular}

Note: Energy Cost $=\$ 13.00 / \mathrm{MBtu}$

Return Pipe Temperature $=150 \mathrm{~F}$ 
TABLE B-12.a

Maximum Allowable Heat Losses (Btu/h'ft) from the Supply

Pipe ( $S$ ) and the Return Pipe (R) for Energy Cost of $\$ 14 / M B t u$

\begin{tabular}{|c|c|c|c|c|c|c|c|c|c|}
\hline \multirow{3}{*}{$\begin{array}{l}\text { Pipe } \\
\text { Size } \\
\text { (inch) }\end{array}$} & \multirow{3}{*}{$\begin{array}{l}\text { Pipe } \\
\text { Type }\end{array}$} & \multicolumn{8}{|c|}{ Process Fluid Temperature (F) } \\
\hline & & 150 & 200 & 250 & 300 & 350 & 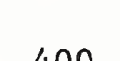 & 50 & 500 \\
\hline & & 120 & $\underline{200}$ & 200 & 200 & 220 & 400 & 430 & 200 \\
\hline 2 & S & 18.6 & 27.6 & 33.6 & 42.8 & 52.8 & 58.3 & 68.1 & 78.2 \\
\hline 1 & $\mathrm{R}$ & 13.4 & 13.3 & 12.1 & 11.9 & 11.7 & 10.9 & $10 \cdot 7$ & 10.6 \\
\hline 3 & S & 21.1 & 31.3 & 42.1 & 53.8 & 60.5 & 72.5 & 78.5 & 90.1 \\
\hline 1.5 & $\mathrm{R}$ & 14.8 & 14.6 & 14.4 & 14.1 & 13.0 & 12.8 & 11.9 & 11.7 \\
\hline 4 & S & 27.7 & 36.6 & 49.4 & 57.2 & 70.6 & 84.5 & 91.0 & 104.6 \\
\hline 2 & $\mathrm{R}$ & 18.3 & 16.3 & 16.1 & 14.7 & 14.4 & 14.2 & .13 .1 & 12.9 \\
\hline 5 & S & 32.0 & 42.2 & 56.9 & 65.6 & 80.9 & 89.0 & 104.0 & 119.5 \\
\hline 2.5 & $\mathrm{R}$ & 20.4 & 18.1 & 17.8 & 16.1 & 15.8 & 14.6 & 14.4 & 14.1 \\
\hline 6 & $s=$ & 32.0 & 47.6 & 57.8 & 73.8 & 83.3 & 92.5 & 101.1 & 109.5 \\
\hline 3 & $\mathrm{R}$ & 20.7 & 20.3 & 18.3 & 17.9 & 16.5 & 15.3 & 14.4 & 13.6 \\
\hline 8 & $S$ & 34.7 & 51.6 & 69.6 & 81.1 & 92.2 & 102.9 & 113.0 & 122.7 \\
\hline 4 & $\mathrm{R}$ & 22.0 & 21.5 & 21.1 & 19.2 & 17.8 & 16.6 & 15.6 & 14.7 \\
\hline 10 & S & 40.6 & 55.1 & 68.4 & 87.4 & 100.0 & 112.1 & 123.4 & 141.9 \\
\hline 6 & $\mathrm{R}$ & 28.2 & 25.5 & 23.3 & 22.8 & 21.1 & 19.6 & 18.3 & 18.0 \\
\hline 12 & $S$ & 46.4 & 62.6 & 77.4 & 91.6 & 105.5 & 118.8 & 138.8 & 159.7 \\
\hline 6 & $\mathrm{R}$ & 28.0 & 25.3 & 23.0 & 21.2 & 19.6 & 18.4 & 18.0 & 17.5 \\
\hline 14 & $S$ & 49.5 & 66.9 & 82.8 & 91.3 & 112.7 & 126.8 & 148.2 & 170.5 \\
\hline 8 & $\mathrm{R}$ & 33.7 & 30.1 & 27.3 & 23.6 & 23.1 & 21.5 & 21.0 & 20.5 \\
\hline 16 & S & 49.6 & 67.7 & 91.5 & 100.8 & 116.6 & 139.7 & 163.2 & 187.9 \\
\hline 8 & $R$ & 30.5 & 27.6 & 26.9 & 23.3 & 21.7 & 21.2 & 20.6 & 20.0 \\
\hline 18 & $S$ & 54.0 & 73.9 & 92.3 & 109.9 & 127.1 & 152.3 & 177.9 & 205.0 \\
\hline 10 & $\mathrm{R}$ & 35.5 & 32.0 & 29.2 & 26.8 & 24.9 & 24.2 & 23.5 & 22.8 \\
\hline
\end{tabular}

Note: Energy Cost $=\$ 14.00 / \mathrm{MBtL}$

Return Pipe Temperature $=150 \mathrm{~F}$ 
TABLE B-12.b

Economic Insulation Thickness (inch) for Steam Supply
and Return Pipes for Energy Cost of $\$ 14 / \mathrm{MBtu}$

\begin{tabular}{|c|c|c|c|c|c|c|c|c|}
\hline \multirow{2}{*}{$\begin{array}{l}\text { Pipe } \\
\text { Size } \\
\text { (inch) }\end{array}$} & \multicolumn{8}{|c|}{ Process Fluid Temperature (F) } \\
\hline & 150 & 200 & 250 & 300 & 350 & 400 & 450 & $\underline{500}$ \\
\hline $2 \& 1$ & 2.5 & 2.5 & 3.0 & 3.0 & 3.0 & 3.5 & 3.5 & 3.5 \\
\hline $3 \& 1.5$ & 3.0 & 3.0 & 3.0 & 3.0 & 3.5 & 3.5 & 4.0 & 4.0 \\
\hline $4 \& 2$ & 2.5 & 3.0 & 3.0 & 3.5 & 3.5 & 3.5 & 4.0 & 4.0 \\
\hline $5 \& 2.5$ & 2.5 & 3.0 & 3.0 & 3.5 & 3.5 & 4.0 & 4.0 & 4.0 \\
\hline $6 \& 3$ & 3.0 & 3.0 & 3.5 & 3.5 & 4.0 & 4.5 & 5.0 & 5.5 \\
\hline $8 \& 4$ & 3.5 & 3.5 & 3.5 & 4.0 & 4.5 & 5.0 & 5.5 & 6.0 \\
\hline $10 \& 6$ & 3.5 & 4.0 & 4.5 & 4.5 & 5.0 & 5.5 & 6.0 & 6.0 \\
\hline $12 \& 6$ & 3.5 & 4.0 & 4.5 & 5.0 & 5.5 & 6.0 & 6.0 & 6.0 \\
\hline $14 \& 8$ & 3.5 & 4.0 & 4.5 & 5.5 & 5.5 & 6.0 & 6.0 & 6.0 \\
\hline $16 \& 8$ & 4.0 & 4.5 & 4.5 & 5.5 & 6.0 & 6.0 & 6.0 & 6.0 \\
\hline $18 \& 10$ & 4.0 & 4.5 & 5.0 & 5.5 & 6.0 & 6.0 & 6.0 & 6.0 \\
\hline
\end{tabular}

Note: Energy Cost $=\$ 14.00 / \mathrm{MBtu}$

Return Pipe Temperature $=150 \mathrm{~F}$ 
TABLE $B-13 \cdot a$

Maximum Allowable Heat Losses ( $B t u / h \cdot f t$ ) from the Supply

Pipe (S), and the Return Pipe (R) for Energy Cost of $\$ 15 / \mathrm{MB}$ tu

\begin{tabular}{|c|c|c|c|c|c|c|c|c|c|}
\hline \multirow{3}{*}{$\begin{array}{c}\text { Pipe } \\
\text { Size } \\
\text { (inch) }\end{array}$} & \multirow{3}{*}{$\begin{array}{l}\text { Pipe } \\
\text { Type }\end{array}$} & \multicolumn{8}{|c|}{ Process Fluid Temperature (F) } \\
\hline & & & & & & & & & \\
\hline & & 150 & $\underline{200}$ & 250 & 300 & 350 & 400 & 450 & $\underline{500}$ \\
\hline 2 & $\mathrm{~s}$ & 18.6 & 27.6 & 33.6 & 42.8 & 52.8 & 58.3 & 68.1 & 78.2 \\
\hline 1 & $\mathbf{R}$ & 13.4 & 13.3 & 12.1 & 11.9 & 11.7 & 10.9 & 10.7 & 10.6 \\
\hline 3 & S & 21.1 & 31.3 & 42.1 & 53.8 & 60.5 & 72.5 & 78.5 & 90.1 \\
\hline 1.5 & $\mathbf{R}$ & 14.8 & 14.6 & 14.4 & 14.1 & 13.0 & 12.8 & 11.9 & 11.7 \\
\hline 4 & $S$ & 27.7 & 36.6 & 49.4 & 57.2 & 70.6 & 84.5 & 91.0 & 104.6 \\
\hline 2 & $\mathrm{R}$ & 18.3 & 16.3 & 16.1 & 14.7 & 14.4 & 14.2 & 13.1 & 12.9 \\
\hline 5 & $S$ & 32.0 & 42.2 & 56.9 & 65.6 & 80.9 & 89.0 & 104.0 & 119.5 \\
\hline 2.5 & $\mathrm{R}$ & 20.4 & 18.1 & 17.8 & 16.1 & 15.8 & 14.6 & 14.4 & 14.1 \\
\hline 6 & $S$ & 32.0 & 47.6 & 57.8 & 67.6 & 77.2 & 86.5 & 95.3 & 103.9 \\
\hline 3 & $\mathrm{R}$ & 20.7 & 20.3 & 18.3 & 16.8 & 15.6 & 14.6 & 13.8 & 13.1 \\
\hline 8 & S & 34.7 & 51.6 & 69.6 & 81.1 & 92.2 & 102.9 & 113.0 & 122.7 \\
\hline 4 & $\mathrm{R}$ & 22.0 & 21.5 & 21.1 & 19.2 & 17.8 & 16.6 & 15.6 & 14.7 \\
\hline 10 & $S$ & 40.6 & 55.1 & 68.4 & 87.4 & 100.0 & 112.1 & 123.4 & 141.9 \\
\hline 6 & $\mathrm{R}$ & 28.2 & 25.5 & 23.3 & 22.8 & 21.1 & 19.6 & 18.3 & 18.0 \\
\hline 12 & $S$ & 46.4 & 62.6 & 77.4 & 91.6 & 105.5 & 118.8 & 138.8 & 159.7 \\
\hline 6 & $\mathbf{R}$ & 28.0 & 25.3 & 23.0 & 21.2 & 19.6 & 18.4 & 18.0 & 17.5 \\
\hline 14 & $S$ & 49.5 & 66.9 & 82.8 & 91.3 & 112.7 & 126.8 & 148.2 & 170.5 \\
\hline 8 & $\mathrm{R}$ & 33.7 & 30.1 & 27.3 & 23.6 & 23.1 & 21.5 & 21.0 & 20.5 \\
\hline 16 & S & 49.6 & 67.7 & 91.5 & 100.8 & 116.6 & 139.7 & 163.2 & 187.9 \\
\hline 8 & $\mathrm{R}$ & 30.5 & 27.6 & 26.9 & 23.3 & 21.7 & 21.2 & 20.6 & 20.0 \\
\hline 18 & $S$ & 54.0 & 73.9 & 92.3 & 109.9 & 127.1 & 152.3 & 177.9 & 205.0 \\
\hline 10 & $\mathbf{R}$ & 35.5 & 32.0 & 29.2 & 26.8 & 24.9 & 24.2 & 23.5 & 22.8 \\
\hline
\end{tabular}


TABLE B-13.b

$$
\begin{gathered}
\text { Economic Insulation Thickness (inch) for Steam Supply } \\
\text { and Return Pipes for Energy Cost of } \$ 15 / \mathrm{MBtu}
\end{gathered}
$$

\begin{tabular}{|c|c|c|c|c|c|c|c|c|}
\hline \multirow{2}{*}{$\begin{array}{l}\text { Pipe } \\
\text { Size } \\
\text { (inch) } \\
\end{array}$} & \multicolumn{8}{|c|}{ Process Fluid Temperature (F) } \\
\hline & $\underline{150}$ & $\underline{200}$ & $\underline{250}$ & $\underline{300}$ & $\underline{350}$ & 400 & $\underline{450}$ & $\underline{500}$ \\
\hline $2 \& 1$ & 2.5 & 2.5 & 3.0 & 3.0 & 3.0 & 3.5 & 3.5 & 3.5 \\
\hline $3 \& 1.5$ & 3.0 & 3.0 & 3.0 & 3.0 & 3.5 & 3.5 & 4.0 & 4.0 \\
\hline $4 \& 2$ & 2.5 & 3.0 & 3.0 & 3.5 & 3.5 & 3.5 & 4.0 & 4.0 \\
\hline $5 \& 2.5$ & 2.5 & 3.0 & 3.0 & 3.5 & 3.5 & 4.0 & 4.0 & 4.0 \\
\hline $6 \& 3$ & 3.0 & 3.0 & 3.5 & 4.0 & 4.5 & 5.0 & 5.5 & 6.0 \\
\hline $8 \& 4$ & 3.5 & 3.5 & 3.5 & 4.0 & 4.5 & 5.0 & 5.5 & 6.0 \\
\hline $10 \& 6$ & 3.5 & 4.0 & 4.5 & 4.5 & 5.0 & 5.5 & 6.0 & 6.0 \\
\hline $12 \& 6$ & -3.5 & 4.0 & 4.5 & 5.0 & 5.5 & 6.0 & 6.0 & 6.0 \\
\hline $14 \& 8$ & 3.5 & 4.0 & 4.5 & 5.5 & 5.5 & 6.0 & 6.0 & 6.0 \\
\hline $16 \& 8$ & 4.0 & 4.5 & 4.5 & 5.5 & 6.0 & 6.0 & 6.0 & 6.0 \\
\hline $18 \& 10$ & 4.0 & 4.5 & 5.0 & 5.5 & 6.0 & 6.0 & 6.0 & 6.0 \\
\hline
\end{tabular}

Note: Energy Cost $=\$ 15.00 / \mathrm{MBtu}$

Return Pipe Temperature $=150 \mathrm{~F}$ 
TABLE $\quad C-1 . a$

Maximum Allowable Heat Losses (Btu/h.ft) from Hot Water Supply

Pipe (S) and Return Pipe (R) for Energy Cost of $\$ 3 / \mathrm{MBtu}$

\begin{tabular}{|c|c|c|c|c|c|c|c|}
\hline \multirow{3}{*}{$\begin{array}{c}\text { Pipe } \\
\text { Size } \\
\text { (inch) } \\
\end{array}$} & \multirow[b]{2}{*}{ Pipe } & \multicolumn{6}{|c|}{ Process Fluid Temperature (F) } \\
\hline & & & & & & & \\
\hline & Type & $\underline{250}$ & $\underline{300}$ & $\underline{350}$ & 400 & 450 & $\underline{500}$ \\
\hline \multirow[t]{2}{*}{1} & S & 34.8 & 44.6 & 55.1 & 66.1 & 77.3 & 89.1 \\
\hline & $\mathbf{R}$ & 34.8 & 34.5 & 34.3 & 34.0 & 33.7 & 33.4 \\
\hline \multirow[t]{2}{*}{2} & $\mathrm{~s}$ & 49.6 & 53.4 & 65.9 & 69.5 & 81.3 & 93.6 \\
\hline & $\mathbf{R}$ & 49.6 & 41.3 & 40.9 & 35.8 & 35.5 & 35.2 \\
\hline \multirow[t]{2}{*}{3} & $S$ & 53.1 & 68.2 & 84.3 & 87.9 & 92.1 & 106.0 \\
\hline & $\mathrm{R}$ & 53.1 & 52.6 & 52.0 & 45.0 & 40.2 & 39.8 \\
\hline \multirow[t]{2}{*}{4} & $S$ & 62.9 & 69.8 & 86.3 & 103.6 & 107.8 & 124.3 \\
\hline & $\mathrm{R}$ & 62.9 & 53.9 & 53.3 & 52.8 & 46.8 & 46.3 \\
\hline \multirow[t]{2}{*}{5} & $S$ & 73.0 & 80.7 & 99.8 & 119.8 & 124.1 & 143.1 \\
\hline & $\mathbf{R}$ & 73.0 & 62.1 & 61.4 & 60.7 & 53.6 & 53.0 \\
\hline \multirow[t]{2}{*}{6} & $S$ & 82.8 & 91.3 & 112.9 & 135.6 & 140.0 & 161.5 \\
\hline & $\mathrm{R}$ & 82.8 & 70.2 & 69.3 & 68.4 & 60.1 & 59.4 \\
\hline \multirow[t]{2}{*}{8} & s & 100.7 & 110.7 & 137.1 & 144.6 & 151.8 & 175.3 \\
\hline & $\mathrm{R}$ & 100.7 & 84.8 & 73.0 & 66.3 & 65.3 & 64.5 \\
\hline \multirow[t]{2}{*}{10} & $S$ & 101.5 & 130.9 & 141.9 & 170.5 & 178.5 & 206.2 \\
\hline & $\mathbf{R}$ & 101.5 & 100.0 & 86.8 & 85.6 & 76.2 & 75.1 \\
\hline \multirow[t]{2}{*}{12} & $S$ & 115.7 & 149.5 & 161.8 & 194.5 & 202.8 & 234.4 \\
\hline & $\mathbf{R}$ & 115.7 & 113.8 & 98.6 & 97.1 & 85.9 & 84.6 \\
\hline \multirow[t]{2}{*}{14} & S & 124.7 & 161.2 & 173.5 & 208.7 & 217.4 & 251.5 \\
\hline & $\mathbf{R}$ & 124.7 & 122.6 & 105.4 & 103.6 & 91.5 & 90.0 \\
\hline \multirow[t]{2}{*}{16} & $S$ & 137.3 & 154.8 & 191.8 & 205.3 & 240.5 & 251.4 \\
\hline & $\mathrm{R}$ & 137.3 & 117.9 & 115.8 & 102.1 & 100.3 & 90.0 \\
\hline \multirow[t]{2}{*}{18} & $S$ & 149.7 & 169.2 & 186.7 & 224.6 & 263.2 & $275.2^{\circ}$ \\
\hline & $\mathbf{R}$ & 149.7 & 128.5 & 113.0 & 110.9 & 108.8 & 97.6 \\
\hline
\end{tabular}

Note: Energy Cost $=\$ 3.00 / \mathrm{MBtu}$

Return Pipe Temperature $=250 \mathrm{~F}$ 
TABLE B-13.b

Economic Insulation Thickness (inch) for Steam Supply and Return Pipes for Energy Cost of $\$ 15 / M B t u$

\begin{tabular}{|c|c|c|c|c|c|c|c|c|}
\hline \multirow{2}{*}{$\begin{array}{l}\text { Pipe } \\
\text { Size } \\
\text { (inch) }\end{array}$} & \multicolumn{8}{|c|}{ Process Fluid Temperature (F) } \\
\hline & 150 & 200 & 250 & 300 & 350 & 400 & 450 & $\underline{500}$ \\
\hline $2 \& 1$ & 2.5 & 2.5 & 3.0 & 3.0 & 3.0 & 3.5 & 3.5 & 3.5 \\
\hline $3 \& 1.5$ & 3.0 & 3.0 & 3.0 & 3.0 & 3.5 & 3.5 & 4.0 & 4.0 \\
\hline $4 \& 2$ & 2.5 & 3.0 & 3.0 & 3.5 & 3.5 & 3.5 & 4.0 & 4.0 \\
\hline $5 \& 2.5$ & 2.5 & 3.0 & 3.0 & 3.5 & 3.5 & 4.0 & 4.0 & 4.0 \\
\hline $6 \& 3$ & 3.0 & 3.0 & 3.5 & 4.0 & 4.5 & 5.0 & 5.5 & 6.0 \\
\hline $8 \& 4$ & 3.5 & 3.5 & 3.5 & 4.0 & 4.5 & 5.0 & 5.5 & 6.0 \\
\hline $10 \& 6$ & 3.5 & 4.0 & 4.5 & 4.5 & 5.0 & 5.5 & 6.0 & 6.0 \\
\hline $12 \& 6$ & -3.5 & 4.0 & 4.5 & 5.0 & 5.5 & 6.0 & 6.0 & 6.0 \\
\hline $14 \& 8$ & 3.5 & 4.0 & 4.5 & 5.5 & 5.5 & 6.0 & 6.0 & 6.0 \\
\hline $16 \& 8$ & 4.0 & 4.5 & 4.5 & 5.5 & 6.0 & 6.0 & 6.0 & 6.0 \\
\hline $18 \& 10$ & 4.0 & 4.5 & 5.0 & 5.5 & 6.0 & 6.0 & 6.0 & 6.0 \\
\hline
\end{tabular}

Note: Energy Cost $=\$ 15.00 / \mathrm{MBtu}$

Return Pipe Temperature $=150 \mathrm{~F}$ 
TABLE $C-1 . a$

Maximum Allowable Heat Losses (Btu/h. ft) from Hot Water Supply

Pipe (S) and Return Pipe (R) for Energy Cost of $\$ 3 /$ MBtu

\begin{tabular}{|c|c|c|c|c|c|c|c|}
\hline \multirow{3}{*}{$\begin{array}{r}\text { Pipe } \\
\text { Size } \\
\text { (inch) } \\
\end{array}$} & \multirow[b]{2}{*}{ Pipe } & \multicolumn{6}{|c|}{ Process Fluid Temperature (F) } \\
\hline & & & & & & & \\
\hline & Type & $\underline{250}$ & $\underline{300}$ & $\underline{350}$ & 400 & $\underline{450}$ & $\underline{500}$ \\
\hline \multirow[t]{2}{*}{1} & $S$ & 34.8 & 44.6 & 55.1 & 66.1 & 77.3 & 89.1 \\
\hline & $\mathrm{R}$ & 34.8 & 34.5 & 34.3 & 34.0 & 33.7 & 33.4 \\
\hline \multirow[t]{2}{*}{2} & S & 49.6 & 53.4 & 65.9 & 69.5 & 81.3 & 93.6 \\
\hline & $\mathrm{R}$ & 49.6 & 41.3 & 40.9 & 35.8 & 35.5 & 35.2 \\
\hline \multirow[t]{2}{*}{3} & $S$ & 53.1 & 68.2 & 84.3 & 87.9 & 92.1 & 106.0 \\
\hline & $\mathrm{R}$ & 53.1 & 52.6 & 52.0 & 45.0 & 40.2 & 39.8 \\
\hline \multirow[t]{2}{*}{4} & $S$ & 62.9 & 69.8 & 86.3 & 103.6 & 107.8 & 124.3 \\
\hline & $\mathrm{R}$ & 62.9 & 53.9 & 53.3 & 52.8 & 46.8 & 46.3 \\
\hline \multirow[t]{2}{*}{5} & $S$ & 73.0 & 80.7 & 99.8 & 119.8 & 124.1 & 143.1 \\
\hline & $\mathrm{R}$ & 73.0 & 62.1 & 61.4 & 60.7 & 53.6 & 53.0 \\
\hline \multirow[t]{2}{*}{6} & S & 82.8 & 91.3 & 112.9 & 135.6 & 140.0 & 161.5 \\
\hline & $\mathrm{R}$ & 82.8 & 70.2 & 69.3 & 68.4 & 60.1 & 59.4 \\
\hline \multirow[t]{2}{*}{8} & S & 100.7 & 110.7 & 137.1 & 144.6 & 151.8 & 175.3 \\
\hline & $\mathrm{R}$ & 100.7 & 84.8 & 73.0 & 66.3 & 65.3 & 64.5 \\
\hline \multirow[t]{2}{*}{10} & $S$ & 101.5 & 130.9 & 141.9 & 170.5 & 178.5 & 206.2 \\
\hline & $\mathrm{R}$ & 101.5 & 100.0 & 86.8 & 85.6 & 76.2 & 75.1 \\
\hline \multirow[t]{2}{*}{12} & $S$ & 115.7 & 149.5 & 161.8 & 194.5 & 202.8 & 234.4 \\
\hline & $\mathrm{R}$ & 115.7 & 113.8 & 98.6 & 97.1 & 85.9 & 84.6 \\
\hline \multirow[t]{2}{*}{14} & $S$ & 124.7 & 161.2 & 173.5 & 208.7 & 217.4 & 251.5 \\
\hline & $\mathrm{R}$ & 124.7 & 122.6 & 105.4 & 103.6 & 91.5 & 90.0 \\
\hline \multirow[t]{2}{*}{16} & $S$ & 137.3 & 154.8 & 191.8 & 205.3 & 240.5 & 251.4 \\
\hline & $\mathrm{R}$ & 137.3 & 117.9 & 115.8 & 102.1 & 100.3 & 90.0 \\
\hline \multirow[t]{2}{*}{18} & $S$ & 149.7 & 169.2 & 186.7 & 224.6 & 263.2 & $275.2^{\circ}$ \\
\hline & $\mathbf{R}$ & 149.7 & 128.5 & 113.0 & 110.9 & 108.8 & 97.6 \\
\hline
\end{tabular}

Note: Energy Cost $=\$ 3.00 / \mathrm{MBtu}$

Return Pipe Temperature $=250 \mathrm{~F}$ 
TABLE $C-1 . b$

Economic Insulation Thickness (inch) for Supply and Return Pipes for Energy Cost of $\$ 3 / \mathrm{MBtu}$

\begin{tabular}{ccccccc}
$\begin{array}{c}\text { Pipe } \\
\text { Size } \\
\text { (inch) }\end{array}$ & 250 & $\underline{6}$ Process Fluid & Temperature $(F)$ & \\
\cline { 2 - 6 } 1 & 1.5 & 1.5 & 1.5 & 1.5 & 1.5 & 1.5 \\
2 & 1.5 & 2.0 & 2.0 & 2.5 & 2.5 & 2.5 \\
3 & 2.0 & 2.0 & 2.0 & 2.5 & 3.0 & 3.0 \\
4 & 2.0 & 2.5 & 2.5 & 2.5 & 3.0 & 3.0 \\
5 & 2.0 & 2.5 & 2.5 & 2.5 & 3.0 & 3.0 \\
6 & 2.0 & 2.5 & 2.5 & 2.5 & 3.0 & 3.0 \\
8 & 2.0 & 2.5 & 2.5 & 3.0 & 3.5 & 3.5 \\
10 & 2.5 & 2.5 & 3.0 & 3.0 & 3.5 & 3.5 \\
12 & 2.5 & 2.5 & 3.0 & 3.0 & 3.5 & 3.5 \\
14 & 2.5 & 2.5 & 3.0 & 3.0 & 3.5 & 3.5 \\
16 & 2.5 & 3.0 & 3.0 & 3.5 & 3.5 & 4.0 \\
18 & 2.5 & 3.0 & 3.0 & 3.5 & 3.5 & 4.0
\end{tabular}

Note: Energy Cost $=\$ 3.00 / \mathrm{MBtu}$

Return Pipe Temperature $=250 \mathrm{~F}$ 
TABLE $C-2 . a$

Maximum Allowable Heat Losses (Btu/h.ft) from Hot Water Supply

Pipe ( $S$ ) and Return Pipe (R) for Energy Cost of $\$ 4 / \mathrm{MBtu}$

\begin{tabular}{|c|c|c|c|c|c|c|c|}
\hline \multirow{3}{*}{$\begin{array}{c}\text { Pipe } \\
\text { Size } \\
\text { (inch) } \\
\end{array}$} & \multirow[b]{2}{*}{ Pipe } & \multicolumn{6}{|c|}{ Process Fluid Temperature (F) } \\
\hline & & & & & & & \\
\hline & Type & $\underline{250}$ & $\underline{300}$ & $\underline{350}$ & 400 & $\underline{450}$ & $\underline{500}$ \\
\hline \multirow[t]{2}{*}{1} & $S$ & 34.8 & 44.6 & 55.1 & 56.7 & 66.3 & 76.3 \\
\hline & $\mathrm{R}$ & 34.8 & 34.5 & 34.3 & 29.3 & 29.1 & 28.8 \\
\hline \multirow[t]{2}{*}{2} & S & 41.6 & 53.4 & 65.9 & 69.5 & 81.3 & 93.6 \\
\hline & $\mathrm{R}$ & 41.6 & 41.3 & 40.9 & 35.8 & 35.5 & 35.2 \\
\hline \multirow[t]{2}{*}{3} & S & 53.1 & 68.2 & 84.3 & 87.9 & 92.1 & 106.0 \\
\hline & $\mathrm{R}$ & 53.1 & 52.6 & 52.0 & 45.0 & 40.2 & 39.8 \\
\hline \multirow[t]{2}{*}{4} & $S$ & 54.4 & 69.8 & 86.3 & 103.6 & 107.8 & 124.3 \\
\hline & $R$ & 54.4 & 53.9 & 53.3 & 52.8 & 46.8 & 46.3 \\
\hline \multirow[t]{2}{*}{5} & $S$ & 62.8 & 80.7 & 99.8 & 119.8 & 124.1 & 143.1 \\
\hline & $\mathbf{R}$ & 62.8 & 62.1 & 61.4 & 60.7 & 53.6 & 53.0 \\
\hline \multirow[t]{2}{*}{6} & $S$ & 71.0 & 91.3 & 112.9 & 135.6 & 140.0 & 161.5 \\
\hline & $\mathrm{R}$ & 71.0 & 70.2 & 69.3 & 68.4 & 60.1 & $59: 4$ \\
\hline \multirow[t]{2}{*}{8} & S & 86.0 & 110.7 & 120.4 & 144.6 & 151.8 & 175.3 \\
\hline & $\mathrm{R}$ & 86.0 & 84.8 & 74.0 & 73.0 & 65.3 & 64.5 \\
\hline \multirow[t]{2}{*}{10} & S & 101.5 & 130.9 & 141.9 & 152.5 & 178.5 & 187.3 \\
\hline & $\mathrm{R}$ & 101.5 & 100.0 & 86.8 & 77.2 & 76.2 & 69.0 \\
\hline \multirow[t]{2}{*}{12} & $S$ & 115.7 & 130.7 & 144.2 & 173.3 & 183.6 & 212.1 \\
\hline & $\mathrm{R}$ & 115.7 & 100.0 & 88.3 & 87.1 & 78.3 & 77.3 \\
\hline \multirow[t]{2}{*}{14} & $S$ & 124.7 & 140.2 & 154.5 & 185.7 & 196.7 & 227.4 \\
\hline & $\mathrm{R}$ & 124.7 & 107.1 & 94.3 & 92.9 & 83.5 & 82.2 \\
\hline \multirow[t]{2}{*}{16} & $S$ & 119.9 & 154.8 & 170.7 & 185.7 & 217.4 & 230.1 \\
\hline & R & 119.9 & 117.9 & 103.8 & 93.0 & 91.6 & 83.2 \\
\hline \multirow[t]{2}{*}{18} & $S$ & 130.8 & 150.7 & 186.7 & 203.0 & 237.8 & 251.6 \\
\hline & $R$ & 130.8 & 114.9 & 113.0 & 101.1 & 99.4 & 90.2 \\
\hline
\end{tabular}

Note: Energy Cost $=\$ 4.00 / \mathrm{MBtu}$

Return Pipe Temperature $=250 \mathrm{~F}$ 
TABLE $\mathrm{C}-2 \cdot \mathrm{b}$

Economic Insulation Thickness (inch) for Supply and Return Pipes for Energy Cost of $\$ 4 / \mathrm{MBtu}$

\begin{tabular}{ccccccc}
$\begin{array}{c}\text { Pipe } \\
\text { Size } \\
\text { (inch) }\end{array}$ & 250 & Process & Fluid & Temperature (F) \\
\cline { 2 - 7 } 1 & 1.5 & 1.5 & 1.5 & 2.0 & 2.0 & 2.0 \\
2 & 2.0 & 2.0 & 2.0 & 2.5 & 2.5 & 2.5 \\
3 & 2.0 & 2.0 & 2.0 & 2.5 & 3.0 & 3.0 \\
4 & 2.5 & 2.5 & 2.5 & 2.5 & 3.0 & 3.0 \\
5 & 2.5 & 2.5 & 2.5 & 2.5 & 3.0 & 3.0 \\
6 & 2.5 & 2.5 & 2.5 & 2.5 & 3.0 & 3.0 \\
8 & 2.5 & 2.5 & 3.0 & 3.0 & 3.5 & 3.5 \\
10 & 2.5 & 2.5 & 3.0 & 3.5 & 3.5 & 4.0 \\
12 & 2.5 & 3.0 & 3.5 & 3.5 & 4.0 & 4.0 \\
14 & 2.5 & 3.0 & 3.5 & 3.5 & 4.0 & 4.0 \\
16 & 3.0 & 3.0 & 3.5 & 4.0 & 4.0 & 4.5 \\
18 & 3.0 & 3.5 & 3.5 & 4.0 & 4.0 & 4.5
\end{tabular}

Note: Energy Cost $=\$ 4.00 / \mathrm{MBtu}$

Return Pipe Temperature $=250 \mathrm{~F}$ 
TABLE $C-3 . a$

Maximum Allowable Heat Losses (Btu/h.ft) from Hot Water Supply

Pipe (S) and Return Pipe (R) for Energy Cost of $\$ 5 / \mathrm{MBtu}$

\begin{tabular}{|c|c|c|c|c|c|c|c|}
\hline \multirow{3}{*}{$\begin{array}{l}\text { Pipe } \\
\text { Size } \\
\text { (inch) }\end{array}$} & \multirow[b]{2}{*}{ Pipe } & \multicolumn{6}{|c|}{ Process Fluid Temperature (F) } \\
\hline & & & & & & & \\
\hline & Type & $\underline{250}$ & $\underline{300}$ & $\underline{350}$ & $\underline{400}$ & 450 & $\underline{500}$ \\
\hline \multirow[t]{2}{*}{1} & $S$ & 34.8 & 38.3 & 47.3 & 56.7 & 66.3 & 68.2 \\
\hline & $\mathrm{R}$ & 34.8 & 29.7 & 29.5 & 29.3 & 29.1 & 25.9 \\
\hline \multirow[t]{2}{*}{2} & $S$ & 41.6 & 46.9 & 57.9 & 69.5 & 81.3 & 84.5 \\
\hline & $\mathrm{R}$ & 41.6 & 36.3 & 36.1 & 35.8 & 35.5 & 32.0 \\
\hline \multirow[t]{2}{*}{3} & $S$ & 53.1 & 59.3 & 73.3 & 78.7 & 92.1 & 106.0 \\
\hline & $\mathrm{R}$ & 53.1 & 45.8 & 45.4 & 40.5 & 40.2 & 39.8 \\
\hline \multirow[t]{2}{*}{4} & $S$ & 54.4 & 69.8 & 86.3 & 92.2 & 107.8 & 124.3 \\
\hline & $\mathrm{R}$ & 54.4 & 53.9 & 53.3 & 47.2 & 46.8 & 46.3 \\
\hline \multirow[t]{2}{*}{5} & S & 62.8 & 80.7 & 99.8 & 106.1 & 124.1 & 143.1 \\
\hline & $\mathrm{R}$ & 62.8 & 62.1 & 61.4 & 54.1 & 53.6 & 53.0 \\
\hline \multirow[t]{2}{*}{6} & $S$ & 71.0 & 91.3 & 112.9 & 119.6 & 126.2 & 145.5 \\
\hline & $\mathrm{R}$ & 71.0 & 70.2 & 69.3 & 60.8 & 54.6 & 54.1 \\
\hline \multirow[t]{2}{*}{8} & $S$ & 75.7 & 97.3 & 120.4 & 129.8 & 138.5 & 159.7 \\
\hline & $\mathrm{R}$ & 75.7 & 74.9 & 74.0 & 66.0 & 59.9 & 59.3 \\
\hline \multirow[t]{2}{*}{10} & $S$ & 89.1 & 114.7 & 127.0 & 152.5 & 162.5 & 172.1 \\
\hline & $\mathrm{R}$ & 89.1 & 88.0 & 78.1 & 77.2 & 69.8 & 63.8 \\
\hline \multirow[t]{2}{*}{12} & S & 101.4 & 116.6 & 144.2 & 156.9 & 183.6 & 194.5 \\
\hline & $\mathrm{R}$ & 101.4 & 89.5 & 88.3 & 79.3 & 78.3 & 71.4 \\
\hline \multirow[t]{2}{*}{14} & $S$ & 108.6 & 124.8 & 154.5 & 168.1 & 196.7 & 208.3 \\
\hline & R & 108.6 & 95.7 & 94.3 & 84.7 & 83.5 & 76.0 \\
\hline \multirow[t]{2}{*}{16} & $S$ & 106.9 & 137.9 & 154.5 & 170.1 & 199.1 & 230.1 \\
\hline & $\mathrm{R}$ & 106.9 & 105.4 & 94.3 & 85.6 & 84.5 & 83.2 \\
\hline \multirow[t]{2}{*}{18} & $S$ & 116.7 & 150.7 & 168.8 & 185.8 & 217.5 & 232.4 \\
\hline & $\mathrm{R}$ & 116.7 & 114.9 & 102.7 & 93.1 & 91.7 & 84.1 \\
\hline
\end{tabular}

Note: Energy Cost $=\$ 5.00 / \mathrm{MBtu}$

Return Pipe Temperature $=250 \mathrm{~F}$ 
TABLE C-3.b

Economic Insulation Thickness (inch) for Supply and Return Pipes for Energy Cost of $\$ 5 / \mathrm{MBtu}$

$\begin{array}{ccccccc}\begin{array}{c}\text { Pipe } \\ \begin{array}{c}\text { Size } \\ \text { (inch) }\end{array}\end{array} & \begin{array}{l}\text { 250 } \\$\cline { 2 - 6 } 1\end{array} & $\underline{300} & \underline{350} & \underline{400} & \underline{450} & \underline{500} \\ 2 & 1.5 & 2.0 & 2.0 & 2.0 & 2.0 & 2.5 \\ 3 & 2.0 & 2.5 & 2.5 & 2.5 & 2.5 & 3.0 \\ 4 & 2.0 & 2.5 & 2.5 & 3.0 & 3.0 & 3.0 \\ 5 & 2.5 & 2.5 & 2.5 & 3.0 & 3.0 & 3.0 \\ 6 & 2.5 & 2.5 & 2.5 & 3.0 & 3.0 & 3.0 \\ 8 & 3.0 & 3.0 & 3.0 & 3.5 & 4.0 & 4.0 \\ 10 & 3.0 & 3.0 & 3.5 & 3.5 & 4.0 & 4.5 \\ 12 & 3.0 & 3.5 & 3.5 & 4.0 & 4.0 & 4.5 \\ 14 & 3.0 & 3.5 & 3.5 & 4.0 & 4.0 & 4.5 \\ 16 & 3.5 & 3.5 & 4.0 & 4.5 & 4.5 & 4.5 \\ 18 & 3.5 & 3.5 & 4.0 & 4.5 & 4.5 & 5.0\end{array}$

Note: Energy Cost $=\$ 5.00 / \mathrm{MBtu}$

Return Pipe Temperature $=250 \mathrm{~F}$ 
TABLE $\quad$ - $-4 . a$

Maximum Allowable Heat Losses (Btu/h.ft) from Hot Water Supply

Pipe (S) and Return Pipe (R) for Energy Cost of $\$ 6 / \mathrm{MBtu}$

\begin{tabular}{|c|c|c|c|c|c|c|c|}
\hline \multirow{3}{*}{$\begin{array}{c}\text { Pipe } \\
\text { Size } \\
\text { (inch) } \\
\end{array}$} & \multirow[b]{2}{*}{ Pipe } & \multicolumn{6}{|c|}{ Process Fluid Temperature (F) } \\
\hline & & & & & & & \\
\hline & Type & $\underline{250}$ & $\underline{300}$ & $\underline{350}$ & $\underline{400}$ & $\underline{450}$ & $\underline{500}$ \\
\hline \multirow[t]{2}{*}{1} & $S$ & 29.9 & 38.3 & 47.3 & 50.7 & 59.3 & 68.2 \\
\hline & $\mathbf{R}$ & 29.9 & 29.7 & 29.5 & 26.3 & 26.1 & 25.9 \\
\hline \multirow[t]{2}{*}{2} & S & 36.6 & 46.9 & 57.9 & 62.8 & 73.5 & 84.5 \\
\hline & $\mathbf{R}$ & 36.6 & 36.3 & 36.1 & 32.5 & 32.3 & 32.0 \\
\hline \multirow[t]{2}{*}{3} & S & 46.2 & 59.3 & 65.6 & 78.7 & 92.1 & 106.0 \\
\hline & $\mathrm{R}$ & 46.2 & 45.8 & 40.8 & 40.5 & 40.2 & 39.8 \\
\hline \multirow[t]{2}{*}{4} & $\mathrm{~S}$ & 54.4 & 69.8 & 76.8 & 92.2 & 107.8 & 112.8 \\
\hline & $\mathrm{R}$ & 54.4 & 53.9 & 47.7 & 47.2 & 46.8 & 42.4 \\
\hline \multirow[t]{2}{*}{5} & $S$ & 62.8 & 71.5 & 88.4 & 106.1 & 124.1 & 129.4 \\
\hline & $\mathrm{R}$ & 62.8 & 55.2 & 54.7 & 54.1 & 53.6 & 48.3 \\
\hline \multirow[t]{2}{*}{6} & $S$ & 71.0 & 80.6 & 99.7 & 119.6 & 126.2 & 145.1 \\
\hline & $\mathrm{R}$ & 71.0 & 62.2 & 61.5 & 60.8 & 54.6 & 54.1 \\
\hline \multirow[t]{2}{*}{8} & $S$ & 75.7 & 87.4 & 108.1 & 129.8 & 138.5 & 159.7 \\
\hline & $\mathrm{R}$ & 75.7 & 67.4 & 66.7 & 66.0 & 59.9 & 59.3 \\
\hline \multirow[t]{2}{*}{10} & $S$ & 89.1 & 102.7 & 127.0 & 138.7 & 149.2 & 172.1 \\
\hline & $\mathrm{R}$ & 89.1 & 79.0 & 78.1 & 70.6 & 64.5 & 63.8 \\
\hline \multirow[t]{2}{*}{12} & $s$ & 90.6 & 116.6 & 130.7 & 156.9 & 168.5 & 180.2 \\
\hline & $\mathrm{R}$ & 90.6 & 89.5 & 80.3 & 79.3 & 72.3 & 66.6 \\
\hline \multirow[t]{2}{*}{14} & $\mathrm{~S}$ & 96.9 & 124.8 & 139.9 & 168.1 & 180.3 & 192.8 \\
\hline & R & 96.9 & 95.7 & 85.8 & 84.7 & 77.0 & 70.9 \\
\hline \multirow[t]{2}{*}{16} & $S$ & 106.9 & 124.8 & 141.6 & 170.1 & 199.1 & 212.7 \\
\hline & $\mathrm{R}$ & 106.9 & 95.7 & 86.8 & 85.6 & 84.5 & 77.6 \\
\hline \multirow[t]{2}{*}{18} & $S$ & 116.7 & 136.4 & 154.6 & 171.8 & 201.1 & 216.5 \\
\hline & $\mathrm{R}$ & 116.7 & 104.3 & 94.5 & 86.5 & 85.4 & 78.9 \\
\hline
\end{tabular}

Note: Energy Cost $=\$ 6.00 / \mathrm{MBtu}$

Return Pipe Temperature $=250 \mathrm{~F}$ 


$$
\text { TABLE } c-4 \cdot b
$$

Economic Insulation Thickness (inch) for Supply and Return Pipes for Energy Cost of $\$ 6 / \mathrm{MBtu}$

\begin{tabular}{|c|c|c|c|c|c|c|}
\hline \multirow{2}{*}{$\begin{array}{c}\text { Pipe } \\
\text { Size } \\
\text { (inch) } \\
\end{array}$} & \multicolumn{6}{|c|}{ Process Fluid Temperature (F) } \\
\hline & $\underline{250}$ & $\underline{300}$ & $\underline{350}$ & 400 & 450 & $\underline{500}$ \\
\hline 1 & 2.0 & 2.0 & 2.0 & 2.5 & 2.5 & 2.5 \\
\hline 2 & 2.5 & 2.5 & 2.5 & 3.0 & 3.0 & 3.0 \\
\hline 3 & 2.5 & 2.5 & 3.0 & 3.0 & 3.0 & 3.0 \\
\hline 4 & 2.5 & 2.5 & 3.0 & 3.0 & 3.0 & 3.5 \\
\hline 5 & 2.5 & 3.0 & 3.0 & 3.0 & 3.0 & 3.5 \\
\hline 6 & 2.5 & 3.0 & 3.0 & 3.0 & 3.5 & 3.5 \\
\hline 8 & 3.0 & 3.5 & 3.5 & 3.5 & 4.0 & 4.0 \\
\hline-10 & 3.0 & 3.5 & 3.5 & 4.0 & 4.5 & 4.5 \\
\hline 12 & 3.5 & 3.5 & 4.0 & 4.0 & 4.5 & 5.0 \\
\hline 14 & 3.5 & 3.5 & 4.0 & 4.0 & 4.5 & 5.0 \\
\hline 16 & 3.5 & 4.0 & 4.5 & 4.5 & 4.5 & 5.0 \\
\hline 18 & 3.5 & 4.0 & 4.5 & 5.0 & 5.0 & 5.5 \\
\hline
\end{tabular}

Note: Energy Cost $=\$ 6.00 / \mathrm{MBtu}$

Return Pipe Temperature $=250 \mathrm{~F}$ 
TABLE $C-5 . a$

Maximum Allowable Heat Losses (Btu/h.ft) from Hot Water Supply Pipe (S) and Return Pipe (R) for Energy Cost of $\$ 7 / \mathrm{MBtu}$

\begin{tabular}{|c|c|c|c|c|c|c|c|}
\hline \multirow{2}{*}{$\begin{array}{c}\text { Pipe } \\
\text { Size } \\
\text { (inch) }\end{array}$} & \multirow[b]{2}{*}{$\begin{array}{l}\text { Pipe } \\
\text { Type }\end{array}$} & \multicolumn{6}{|c|}{ Process Fluid Temperature (E) } \\
\hline & & $\underline{250}$ & $\underline{300}$ & 350 & 400 & 450 & $\underline{500}$ \\
\hline \multirow[t]{2}{*}{1} & 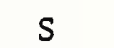 & 29.9 & 38.3 & 47.3 & 50.7 & 59.3 & 68.2 \\
\hline & $\mathbf{R}$ & 29.9 & 29.7 & 29.5 & 26.3 & 26.1 & 25.9 \\
\hline \multirow[t]{2}{*}{2} & S & 36.6 & 46.9 & 57.9 & 62.8 & 73.5 & 84.5 \\
\hline & $\mathrm{R}$ & 36.6 & 36.3 & 36.1 & 32.5 & 32.3 & 32.0 \\
\hline \multirow[t]{2}{*}{3} & $S$ & 46.2 & 53.1 & 65.6 & 78.7 & 92.1 & 106.0 \\
\hline & $\mathbf{R}$ & 46.2 & 41.1 & 40.8 & 40.5 & 40.2 & 39.8 \\
\hline \multirow[t]{2}{*}{4} & S & 54.4 & 62.2 & 76.8 & 92.2 & 98.0 & 112.8 \\
\hline & $\mathrm{R}$ & 54.4 & 48.1 & 47.7 & 47.2 & 42.8 & 42.4 \\
\hline \multirow[t]{2}{*}{5} & S & 62.8 & 71.5 & 88.4 & 106.1 & 112.3 & 129.4 \\
\hline & $\mathrm{R}$ & 62.8 & 55.2 & 54.7 & 54.1 & 48.8 & 48.3 \\
\hline \multirow[t]{2}{*}{6} & $S$ & 71.0 & 80.6 & 99.7 & 107.9 & 115.6 & 133.2 \\
\hline & $\mathrm{R}$ & 71.0 & 62.2 & 61.5 & 55.2 & $50 . .3$ & 49.9 \\
\hline \multirow[t]{2}{*}{8} & $S$ & 75.7 & 87.4 & 108.1 & 118.4 & 127.9 & 147.4 \\
\hline & $\mathrm{R}$ & 75.7 & 67.4 & 66.7 & 66.5 & 55.7 & 55.1 \\
\hline \multirow[t]{2}{*}{10} & $S$ & 79.9 & 102.7 & 115.5 & 127.6 & 149.2 & 159.7 \\
\hline & $\mathrm{R}$ & 79.9 & 79.0 & 71.4 & 65.2 & 64.5 & 59.5 \\
\hline \multirow[t]{2}{*}{12} & $S$ & 82.1 & 105.6 & 130.7 & 144.0 & 156.2 & 168.4 \\
\hline & $\mathrm{R}$ & 82.1 & 81.3 & 80.3 & 73.1 & 67.3 & 62.5 \\
\hline \multirow[t]{2}{*}{14} & S & 87.9 & 113.1 & 139.9 & 154.1 & 167.0 & 180.0 \\
\hline & $\mathrm{R}$ & 87.9 & 86.9 & 85.8 & 78.0 & 71.7 & 66.5 \\
\hline \multirow[t]{2}{*}{16} & $S$ & 96.9 & 114.4 & 141.6 & 170.1 & 184.1 & 198.4 \\
\hline & $\mathbf{R}$ & 96.9 & 87.9 & 86.8 & 85.6 & 78.6 & 72.8 \\
\hline \multirow[t]{2}{*}{18} & $S$ & 105.7 & 124.9 & 143.0 & 171.8 & 187.4 & 216.5 \\
\hline & $\mathrm{R}$ & 105.7 & 95.8 & 87.7 & 86.5 & 80.0 & 78.9 \\
\hline
\end{tabular}


TABLE $C-5 . b$

Economic Insulation Thickness (inch) for Supply
and Return Pipes for Energy Cost of $\$ 7 / \mathrm{MBtu}$

\begin{tabular}{|c|c|c|c|c|c|c|}
\hline \multirow{2}{*}{$\begin{array}{c}\text { Pipe } \\
\text { Size } \\
\text { (inch) }\end{array}$} & \multicolumn{6}{|c|}{ Process Fluid Temperature (F) } \\
\hline & $\underline{250}$ & $\underline{300}$ & $\underline{350}$ & 400 & 450 & $\underline{500}$ \\
\hline 1 & 2.0 & 2.0 & 2.0 & 2.5 & 2.5 & 2.5 \\
\hline 2 & 2.5 & 2.5 & 2.5 & 3.0 & 3.0 & 3.0 \\
\hline 3 & 2.5 & 3.0 & 3.0 & 3.0 & 3.0 & 3.0 \\
\hline 4 & 2.5 & 3.0 & 3.0 & 3.0 & 3.5 & 3.5 \\
\hline 5 & 2.5 & 3.0 & 3.0 & 3.0 & 3.5 & 3.5 \\
\hline 6 & 2.5 & 3.0 & 3.0 & 3.5 & 4.0 & 4.0 \\
\hline 8 & 3.0 & 3.5 & 3.5 & 4.0 & 4.5 & 4.5 \\
\hline-10 & 3.5 & 3.5 & 4.0 & 4.5 & 4.5 & 5.0 \\
\hline 12 & 4.0 & 4.0 & 4.0 & 4.5 & 5.0 & 5.5 \\
\hline 14 & 4.0 & 4.0 & 4.0 & 4.5 & 5.0 & 5.5 \\
\hline 16 & 4.0 & 4.5 & 4.5 & 4.5 & 5.0 & 5.5 \\
\hline 18 & 4.0 & 4.5 & 5.0 & 5.0 & 5.5 & 5.5 \\
\hline
\end{tabular}

Note: Energy Cost $=\$ 7.00 / \mathrm{MBtu}$

Return Pipe Temperature $=250 \mathrm{~F}$ 
TABLE $C-6 . a$

Maximum Allowable Heat Losses (Btu/h.ft) from Hot Water Supply Pipe ( $S$ ) and Return Pipe (R) for Energy Cost of $\$ 8 / \mathrm{MBtu}$

\begin{tabular}{|c|c|c|c|c|c|c|c|}
\hline \multirow{3}{*}{$\begin{array}{c}\text { Pipe } \\
\text { Size } \\
\text { (inch) }\end{array}$} & \multirow[b]{2}{*}{ Pipe } & \multicolumn{6}{|c|}{ Process Fluid Temperature (F) } \\
\hline & & & & & & & \\
\hline & Type & $\underline{250}$ & $\underline{300}$ & $\underline{350}$ & 400 & $\underline{450}$ & $\underline{500}$ \\
\hline \multirow[t]{2}{*}{1} & $S$ & 29.9 & 34.2 & 42.3 & 50.7 & 59.3 & 62.5 \\
\hline & $\mathrm{R}$ & 29.9 & 26.6 & 26.4 & 26.3 & 26.1 & 23.9 \\
\hline \multirow[t]{2}{*}{2} & S & 36.6 & 42.4 & 52.4 & 62.8 & 73.5 & 84.5 \\
\hline & $\mathrm{R}$ & 36.6 & 32.9 & 32.7 & 32.5 & 32.3 & 32.0 \\
\hline \multirow[t]{2}{*}{3} & $\mathrm{~S}$ & 41.4 & 53.1 & 65.6 & 78.7 & 92.1 & 106.0 \\
\hline & $\mathrm{R}$ & 41.4 & 41.1 & 40.8 & 40.5 & 40.2 & 39.8 \\
\hline \multirow[t]{2}{*}{4} & $S$ & 48.5 & 62.2 & 69.8 & 83.8 & 98.0 & 112.8 \\
\hline & $\mathrm{R}$ & 48.5 & 48.1 & 43.5 & 43.1 & 42.8 & 42.4 \\
\hline \multirow[t]{2}{*}{5} & $S$ & 55.7 & 71.5 & 80.0 & 96.0 & 112.3 & 118.8 \\
\hline & $\mathrm{R}$ & 55.7 & 55.2 & 49.7 & 49.2 & 48.8 & 44.7 \\
\hline \multirow[t]{2}{*}{6} & $S$ & 62.8 & 72.7 & 89.9 & 107.9 & 115.6 & 133.2 \\
\hline & R & 62.8 & 56.2 & 55.7 & 55.2 & 50.3 & 49.9 \\
\hline \multirow[t]{2}{*}{8} & S & 68.1 & 79.8 & 98.7 & 118.4 & -127.9 & 147.4 \\
\hline & $\mathrm{R}$ & 68.1 & 61.7 & 61.1 & 60.5 & 55.7 & 55.1 \\
\hline \multirow[t]{2}{*}{10} & $\mathrm{~S}$ & 79.9 & 93.5 & 106.3 & 127.6 & 149.2 & 159.7 \\
\hline & R & 79.9 & 72.1 & 65.8 & 65.2 & 64.5 & 59.5 \\
\hline \multirow[t]{2}{*}{12} & $S$ & 82.1 & 105.6 & 119.9 & 133.5 & 156.2 & 168.4 \\
\hline & $\mathrm{R}$ & 82.1 & 81.3 & 74.0 & 68.0 & 67.3 & 62.5 \\
\hline \multirow[t]{2}{*}{14} & S & 87.9 & 113.1 & 128.3 & 142.8 & 156.0 & 180.0 \\
\hline & $\mathrm{R}$ & 87.9 & 86.9 & 78.9 & 72.6 & 67.3 & 66.5 \\
\hline \multirow[t]{2}{*}{16} & $S$ & 88.9 & 114.4 & 131.0 & 157.4 & 171.8 & 186.2 \\
\hline & $\mathrm{R}$ & 88.9 & 87.9 & 80.6 & 79.6 & 73.7 & 68.7 \\
\hline \multirow[t]{2}{*}{18} & $S$ & 97.0 & 115.6 & 133.3 & 160.1 & 187.4 & 203.0 \\
\hline & $\mathrm{R}$ & 97.0 & 88.8 & 82.0 & 81.0 & 80.0 & 74.5 \\
\hline
\end{tabular}

Note: Energy Cost $=\$ 8.00 / \mathrm{MBtu}$

Return Pipe Temperature $=250 \mathrm{~F}$ 
TABLE $C-6 \cdot b$

Economic Insulation Thickness (inch) for Supply and Return Pipes for Energy Cost of $\$ 8 / \mathrm{MBtu}$

\begin{tabular}{|c|c|c|c|c|c|c|}
\hline Pipe & & Proce & Elui & nper & $(F)$ & \\
\hline $\begin{array}{c}\text { Size } \\
\text { (inch) }\end{array}$ & $\underline{250}$ & $\underline{300}$ & $\underline{350}$ & 400 & 450 & $\underline{500}$ \\
\hline 1 & 2.0 & 2.5 & 2.5 & 2.5 & 2.5 & 3.0 \\
\hline 2 & 2.5 & 3.0 & 3.0 & 3.0 & 3.0 & 3.0 \\
\hline 3 & 3.0 & 3.0 & 3.0 & 3.0 & 3.0 & 3.0 \\
\hline 4 & 3.0 & 3.0 & 3.5 & 3.5 & 3.5 & 3.5 \\
\hline 5 & 3.0 & 3.0 & 3.5 & 3.5 & 3.5 & 4.0 \\
\hline 6 & 3.0 & 3.5 & 3.5 & 3.5 & 4.0 & 4.0 \\
\hline 8 & 3.5 & 4.0 & 4.0 & 4.0 & 4.5 & 4.5 \\
\hline-10 & 3.5 & 4.0 & 4.5 & 4.5 & 4.5 & 5.0 \\
\hline 12 & 4.0 & 4.0 & 4.5 & 5.0 & 5.0 & 5.5 \\
\hline 14 & 4.0 & 4.0 & 4.5 & 5.0 & 5.5 & 5.5 \\
\hline 16 & 4.5 & 4.5 & 5.0 & 5.0 & 5.5 & 6.0 \\
\hline 18 & 4.5 & 5.0 & 5.5 & 5.5 & 5.5 & 6.0 \\
\hline
\end{tabular}

Note: Energy Cost $=\$ 8.00 / \mathrm{MBtu}$

Return Pipe Temperature $=250 \mathrm{~F}$ 
TABLE $C-7 \cdot a$

Maximum Allowable Heat Losses (Btu/h.ft) from Hot Water Supply Pipe (S) and Return Pipe (R) for Energy Cost of $\$ 9 / M B t u$

\begin{tabular}{|c|c|c|c|c|c|c|c|}
\hline \multirow{2}{*}{$\begin{array}{l}\text { Pipe } \\
\text { Size }\end{array}$} & \multirow[b]{2}{*}{ Pipe } & \multicolumn{6}{|c|}{ Process Fluid Temperature (F) } \\
\hline & & & & & & & \\
\hline & Type & $\underline{250}$ & $\underline{300}$ & $\underline{350}$ & 400 & $\underline{450}$ & $\underline{500}$ \\
\hline \multirow[t]{2}{*}{1} & $S$ & 26.8 & 34.2 & 42.3 & 46.5 & 54.4 & 62.5 \\
\hline & $\mathrm{R}$ & 26.8 & 26.6 & 26.4 & 24.2 & 24.0 & 23.9 \\
\hline \multirow[t]{2}{*}{2} & S & 36.6 & 42.4 & 52.4 & 62.8 & 73.5 & 84.5 \\
\hline & $\mathrm{R}$ & 36.6 & 32.9 & 32.7 & 32.5 & 32.3 & 32.0 \\
\hline \multirow[t]{2}{*}{3} & $S$ & 41.4 & 53.1 & 65.6 & 78.7 & 84.1 & 96.8 \\
\hline & $\mathrm{R}$ & 41.4 & 41.1 & 40.8 & 40.5 & 36.9 & 36.6 \\
\hline \multirow[t]{2}{*}{4} & S & 48.5 & 62.2 & 69.8 & 83.8 & 98.0 & 112.8 \\
\hline & $\mathrm{R}$ & 48.5 & 48.1 & 43.5 & 43.1 & 42.8 & 42.4 \\
\hline \multirow[t]{2}{*}{5} & $S$ & 55.7 & 71.5 & 80.0 & 96.0 & 103.2 & 118.8 \\
\hline & $\mathrm{R}$ & 55.7 & 55.2 & 49.7 & 49.2 & 45.1 & 44.7 \\
\hline \multirow[t]{2}{*}{6} & $\mathrm{~S}$ & 62.8 & 72.7 & 89.9 & 107.9 & 115.6 & 123.5 \\
\hline & $\mathrm{R}$ & 62.8 & 56.2 & 55.7 & 55.2 & 50.3 & 46.5 \\
\hline \multirow[t]{2}{*}{8} & $\mathrm{~s}$ & 68.1 & 79.8 & 98.7 & 109.4 & 127.9 & 137.4 \\
\hline & $\mathrm{R}$ & 68.1 & 61.7 & 61.1 & 56.2 & 55.7 & 55.7 \\
\hline \multirow[t]{2}{*}{10} & S & 72.8 & 93.5 & 106.3 & 127.6 & 138.6 & 149.5 \\
\hline & R & 72.8 & 72.1 & 65.8 & 65.2 & 60.1 & 55.9 \\
\hline \multirow[t]{2}{*}{12} & $S$ & 82.1 & 97.0 & 111.2 & 133.5 & 146.0 & 168.4 \\
\hline & $\mathrm{R}$ & 82.1 & 74.7 & 68.7 & 68.0 & 63.2 & 62.5 \\
\hline \multirow[t]{2}{*}{14} & $\mathrm{~S}$ & 87.9 & 103.7 & 118.9 & 133.4 & 156.0 & 169.2 \\
\hline & $\mathrm{R}$ & 87.9 & 79.8 & 73.4 & 68.0 & 67.3 & 62.8 \\
\hline \multirow[t]{2}{*}{16} & S & 88.9 & 114.4 & 131.0 & 146.8 & 171.8 & 186.2 \\
\hline & $\mathrm{R}$ & 88.9 & 87.9 & 80.6 & 74.6 & 73.7 & 68.7 \\
\hline \multirow[t]{2}{*}{18} & $\mathrm{~S}$ & 97.0 & 115.6 & 133.3 & 160.1 & 187.4 & 203.0 \\
\hline & R & 97.0 & 88.8 & 82.0 & 81.0 & 80.0 & 74.5 \\
\hline
\end{tabular}


TABLE $c-7 . b$

Economic Insulation Thickness (inch) for Supply and Return Pipes for Energy Cost of $\$ 9 / \mathrm{MBtu}$

\begin{tabular}{ccccccc}
$\begin{array}{c}\text { Pipe } \\
\text { Size } \\
\text { (inch) }\end{array}$ & $\underline{6}$ Process Fluid & Temperature $(F)$ & \\
\cline { 2 - 7 } 1 & 2.5 & 2.5 & 2.5 & 3.0 & 3.0 & 3.0 \\
2 & 2.5 & 3.0 & 3.0 & 3.0 & 3.0 & 3.0 \\
3 & 3.0 & 3.0 & 3.0 & 3.0 & 3.5 & 3.5 \\
4 & 3.0 & 3.0 & 3.5 & 3.5 & 3.5 & 3.5 \\
5 & 3.0 & 3.0 & 3.5 & 3.5 & 4.0 & 4.0 \\
6 & 3.0 & 3.5 & 3.5 & 3.5 & 4.0 & 4.5 \\
8 & 3.5 & 4.0 & 4.0 & 4.5 & 4.5 & 5.0 \\
-10 & 4.0 & 4.0 & 4.5 & 4.5 & 5.0 & 5.5 \\
12 & 4.0 & 4.5 & 5.0 & 5.0 & 5.5 & 5.5 \\
14 & 4.0 & 4.5 & 5.0 & 5.5 & 5.5 & 6.0 \\
16 & 4.5 & 4.5 & 5.0 & 5.5 & 5.5 & 6.0 \\
18 & 4.5 & 5.0 & 5.5 & 5.5 & 5.5 & 6.0
\end{tabular}

Note: Energy Cost $=\$ 9.00 / \mathrm{MBtu}$

Return Pipe Temperature $=250 \mathrm{~F}$ 
TABLE C-8.a

Maximum Allowable Heat Losses (Btu/h.ft) from Hot Water Supply

Pipe (S) and Return Pipe (R) for Energy Cost of $\$ 10 / \mathrm{MBtu}$

\begin{tabular}{|c|c|c|c|c|c|c|c|}
\hline \multirow{2}{*}{$\begin{array}{l}\text { Pipe } \\
\text { Size } \\
\text { (inch) } \\
\end{array}$} & \multirow[b]{2}{*}{$\begin{array}{l}\text { Pipe } \\
\text { Iype }\end{array}$} & \multicolumn{6}{|c|}{ Process Fluid Temperature (F) } \\
\hline & & $\underline{250}$ & $\underline{300}$ & $\underline{350}$ & $\underline{400}$ & $\underline{450}$ & $\underline{500}$ \\
\hline \multirow[t]{2}{*}{1} & S & 26.8 & 34.2 & 38.8 & 46.5 & 54.4 & 62.5 \\
\hline & $\mathrm{R}$ & 26.8 & 26.6 & 24.3 & 24.2 & 24.0 & 23.9 \\
\hline \multirow[t]{2}{*}{2} & S & 36.6 & 42.4 & 52.4 & 62.8 & 73.5 & 77.9 \\
\hline & $\mathrm{R}$ & 36.6 & 32.9 & 32.7 & 32.5 & 32.3 & 29.7 \\
\hline \multirow[t]{2}{*}{3} & $S$ & 41.4 & 53.1 & 65.6 & 78.7 & 84.1 & 96.8 \\
\hline & $\mathrm{R}$ & 41.4 & 41.1 & 40.8 & 40.5 & 36.9 & 36.6 \\
\hline \multirow[t]{2}{*}{4} & S & 48.5 & 56.5 & 69.8 & 83.8 & 98.0 & 112.8 \\
\hline & $\mathrm{R}$ & 48.5 & 43.8 & 43.5 & 43.1 & 42.8 & 42.4 \\
\hline \multirow[t]{2}{*}{5} & S & 55.7 & 64.7 & 80.0 & 88.2 & 103.2 & 118.8 \\
\hline & $\mathrm{R}$ & 55.7 & 50.1 & 49.7 & 45.4 & 45.1 & 44.7 \\
\hline \multirow[t]{2}{*}{6} & $S$ & 56.7 & 72.7 & 89.9 & 98.9 & 107.3 & 123.5 \\
\hline & $\mathrm{R}$ & 56.7 & 56.2 & 55.7 & 50.8 & 46.9 & 46.5 \\
\hline \multirow[t]{2}{*}{8} & $S$ & 62.2 & 73.8 & 91.2 & 109.4 & 119.3 & 137.4 \\
\hline & R & 62.2 & 57.1 & 56.6 & 56.2 & 52.1 & 51.7 \\
\hline \multirow[t]{2}{*}{10} & S & 72.8 & 86.0 & 98.7 & 118.5 & 129.8 & 149.5 \\
\hline & $\mathrm{R}$ & 72.8 & 66.4 & 61.2 & 60.7 & 56.5 & 55.9 \\
\hline \multirow[t]{2}{*}{12} & $S$ & 75.5 & 97.0 & 111.2 & 124.8 & 146.0 & 158.4 \\
\hline & $\mathrm{R}$ & 75.5 & 74.7 & 68.7 & 63.8 & 63.2 & 59.1 \\
\hline \multirow[t]{2}{*}{14} & $S$ & 80.7 & 103.7 & 118.9 & 133.4 & 156.0 & 169.2 \\
\hline & $\mathrm{R}$ & 80.7 & 79.8 & 73.4 & 68.0 & 67.3 & 62.8 \\
\hline \multirow[t]{2}{*}{16} & $S$ & 88.9 & 114.4 & 131.0 & 146.8 & 161.3 & 186.2 \\
\hline & $\mathrm{R}$ & 88.9 & 87.9 & 80.6 & 74.6 & 69.5 & 68.7 \\
\hline \multirow[t]{2}{*}{18} & $S$ & 89.8 & 115.6 & 133.3 & 150.3 & 175.8 & 203.0 \\
\hline & $\mathrm{R}$ & 89.8 & 88.8 & 82.0 & 76.3 & 75.4 & 74.5 \\
\hline
\end{tabular}

Note: Energy Cost $=\$ 10.00 / \mathrm{MBtu}$

Return Pipe Temperature $=250 \mathrm{~F}$ 
TABLE $\mathrm{C}-8 \cdot \mathrm{b}$

Economic Insulation Thickness (inch) for Supply
and Return Pipes for Energy Cost of $\$ 10 /$ MBtu

\begin{tabular}{ccccccc}
$\begin{array}{c}\text { Pipe } \\
\text { Size } \\
\text { (inch) }\end{array}$ & 250 & 300 & $\underline{350}$ & 400 & 450 & $\underline{500}$ \\
\cline { 2 - 6 } 1 & 2.5 & 2.5 & 3.0 & 3.0 & 3.0 & 3.0 \\
2 & 2.5 & 3.0 & 3.0 & 3.0 & 3.0 & 3.5 \\
3 & 3.0 & 3.0 & 3.0 & 3.0 & 3.5 & 3.5 \\
4 & 3.0 & 3.5 & 3.5 & 3.5 & 3.5 & 3.5 \\
5 & 3.0 & 3.5 & 3.5 & 4.0 & 4.0 & 4.0 \\
6 & 3.5 & 3.5 & 3.5 & 4.0 & 4.5 & 4.5 \\
8 & 4.0 & 4.5 & 4.5 & 4.5 & 5.0 & 5.0 \\
-10 & 4.0 & 4.5 & 5.0 & 5.0 & 5.5 & 5.5 \\
12 & 4.5 & 4.5 & 5.0 & 5.5 & 5.5 & 6.0 \\
14 & 4.5 & 4.5 & 5.0 & 5.5 & 5.5 & 6.0 \\
16 & 4.5 & 4.5 & 5.0 & 5.5 & 6.0 & 6.0 \\
18 & 5.0 & 5.5 & 5.5 & 6.0 & 6.0 & 6.0
\end{tabular}

Note: Energy Cost $=\$ 10.00 / \mathrm{MBtu}$

Return Pipe Temperature $=250 \mathrm{~F}$ 
TABLE $C-9 . \mathrm{a}$

Maximum Allowable Heat Losses (Btu/h.ft) from Hot Water Supply Pipe (S) and Return Pipe (R) for Energy Cost of $\$ 11 /$ MBtu

\begin{tabular}{|c|c|c|c|c|c|c|c|}
\hline \multirow{3}{*}{$\begin{array}{c}\text { Pipe } \\
\text { Size } \\
\text { (inch) }\end{array}$} & \multirow[b]{2}{*}{ Pipe } & \multicolumn{6}{|c|}{ Process Fluid Temperature (F) } \\
\hline & & & & & & & \\
\hline & Type & $\underline{250}$ & $\underline{300}$ & $\underline{350}$ & $\underline{400}$ & $4 \underline{50}$ & $\underline{500}$ \\
\hline \multirow[t]{2}{*}{1} & S & 26.8 & 34.2 & 38.8 & 46.5 & 54.4 & 62.5 \\
\hline & $\mathbf{R}$ & 26.8 & 26.6 & 24.3 & 24.2 & 24.0 & 23.9 \\
\hline \multirow[t]{2}{*}{2} & $S$ & 33.1 & 42.4 & 52.4 & 62.8 & 67.7 & 77.9 \\
\hline & $\mathrm{R}$ & 33.1 & 32.9 & 32.7 & 32.5 & 29.9 & 29.7 \\
\hline \multirow[t]{2}{*}{3} & $S$ & 41.4 & 53.1 & 65.6 & 78.7 & 84.1 & 96.8 \\
\hline & $\mathbf{R}$ & 41.4 & 41.1 & 40.8 & 40.5 & 36.9 & 36.6 \\
\hline \multirow[t]{2}{*}{4} & $S$ & 44.1 & 56.5 & 69.8 & 83.8 & 92.8 & 112.8 \\
\hline & $\mathbf{R}$ & 44.1 & 43.8 & 43.5 & 43.1 & 42.8 & 42.4 \\
\hline \multirow[t]{2}{*}{5} & S & 50.5 & 64.7 & 73.5 & 88.2 & 103.2 & 118.8 \\
\hline & $\mathbf{R}$ & 50.5 & 50.1 & 45.8 & 45.4 & 45.1 & 44.7 \\
\hline \multirow[t]{2}{*}{6} & $S$ & 56.7 & 72.7 & 82.4 & 98.9 & 107.3 & 123.5 \\
\hline & $\mathrm{R}$ & 56.7 & 56.2 & 51.2 & 50.8 & 46.9 & 46.5 \\
\hline \multirow[t]{2}{*}{8} & S & 62.2 & 73.8 & 91.2 & 102.0 & 119.3 & 129.0 \\
\hline & $\mathrm{R}$ & 62.2 & 57.1 & 56.6 & 52.5 & 52.1 & 48.7 \\
\hline \multirow[t]{2}{*}{10} & $S$ & 72.8 & 86.0 & 98.7 & 110.9 & 129.8 & 140.9 \\
\hline & $\mathrm{R}$ & 72.8 & 66.4 & 61.2 & 57.0 & 56.5 & 52.9 \\
\hline \multirow[t]{2}{*}{12} & $S$ & 75.5 & 90.0 & 104.0 & 124.8 & 137.4 & 158.4 \\
\hline & $\mathbf{R}$ & 75.5 & 69.4 & 64.4 & 63.8 & 59.7 & 59.1 \\
\hline \multirow[t]{2}{*}{14} & $S$ & 80.7 & 96.2 & 111.1 & 133.4 & 146.7 & 169.2 \\
\hline & $\mathbf{R}$ & 80.7 & 74.1 & 68.7 & 68.0 & 63.5 & 62.8 \\
\hline \multirow[t]{2}{*}{16} & $S$ & 82.4 & 105.9 & 122.3 & 137.9 & 161.3 & 186.2 \\
\hline & $\mathrm{R}$ & 82.4 & 81.5 & 75.4 & 70.3 & 69.5 & 68.7 \\
\hline \multirow[t]{2}{*}{18} & $S$ & 83.8 & 107.8 & 133.3 & 150.3 & 175.8 & 203.0 \\
\hline & $\mathbf{R}$ & 83.8 & 82.9 & 82.0 & 76.3 & 75.4 & 74.5 \\
\hline
\end{tabular}

Note: Energy Cost $=\$ 11.00 / \mathrm{MBtu}$

Return Pipe Temperature $=250 \mathrm{~F}$ 
TABLE $C-9 . b$

Economic Insulation Thickness (inch) for Supply and Return Pipes for Energy Cost of $\$ 11 / \mathrm{MBtu}$

\begin{tabular}{lllllll}
$\begin{array}{c}\text { Pipe } \\
\text { Size } \\
\text { (inch) }\end{array}$ & \multicolumn{6}{c}{ Process Fluid Temperature (F) } \\
\cline { 2 - 7 } 1 & 250 & $\underline{300}$ & $\underline{350}$ & $\underline{400}$ & $\underline{450}$ & $\underline{500}$ \\
2 & 3.0 & 3.0 & 3.0 & 3.0 & 3.5 & 3.5 \\
3 & 3.0 & 3.0 & 3.0 & 3.0 & 3.5 & 3.5 \\
4 & 3.5 & 3.5 & 3.5 & 3.5 & 3.5 & 3.5 \\
5 & 3.5 & 3.5 & 4.0 & 4.0 & 4.0 & 4.0 \\
6 & 3.5 & 3.5 & 4.0 & 4.0 & 4.5 & 4.5 \\
8 & 4.0 & 4.5 & 4.5 & 5.0 & 5.0 & 5.5 \\
10 & 4.0 & 4.5 & 5.0 & 5.5 & 5.5 & 6.0 \\
12 & 4.5 & 5.0 & 5.5 & 5.5 & 6.0 & 6.0 \\
14 & 4.5 & 5.0 & 5.5 & 5.5 & 6.0 & 6.0 \\
16 & 5.0 & 5.0 & 5.5 & 6.0 & 6.0 & 6.0 \\
18 & 5.5 & 5.5 & 5.5 & 6.0 & 6.0 & 6.0
\end{tabular}

Note: Energy Cost $=\$ 11.00 / \mathrm{MBtu}$

Return Pipe Temperature $=250 \mathrm{~F}$ 
TABLE $C-10 . a$

Maximum Allowable Heat Losses (Btu/h.ft) from Hot Water Supply

Pipe (S) and Return Pipe (R) for Energy Cost of $\$ 12 / \mathrm{MB}$ tu

\begin{tabular}{|c|c|c|c|c|c|c|c|}
\hline \multirow{3}{*}{$\begin{array}{c}\text { Pipe } \\
\text { Size } \\
\text { (inch) }\end{array}$} & \multirow[b]{2}{*}{ Pipe } & \multicolumn{6}{|c|}{ Process Eluid Temperature (E) } \\
\hline & & & & & & & \\
\hline & Type & $\underline{250}$ & $\underline{300}$ & $\underline{350}$ & 400 & $\underline{450}$ & $\underline{500}$ \\
\hline \multirow[t]{2}{*}{1} & S & 24.6 & 31.4 & 38.8 & 46.5 & 54.4 & 62.5 \\
\hline & $\mathrm{R}$ & 24.6 & 24.4 & 24.3 & 24.2 & 24.0 & 23.9 \\
\hline \multirow[t]{2}{*}{2} & S & 33.1 & 42.4 & 52.4 & 57.9 & 67.7 & 77.9 \\
\hline & $\mathrm{R}$ & 33.1 & 32.9 & 32.7 & 30.0 & 29.9 & 29.7 \\
\hline \multirow[t]{2}{*}{3} & s & 41.4 & 53.1 & 65.6 & 71.9 & 84.1 & 89.7 \\
\hline & $\mathrm{R}$ & 41.4 & 41.1 & 40.8 & 37.2 & 36.9 & 34.1 \\
\hline \multirow[t]{2}{*}{4} & S & 44.1 & 56.5 & 69.8 & 83.8 & 98.0 & 104.0 \\
\hline & $\mathrm{R}$ & 44.1 & 43.8 & 43.5 & 43.1 & 42.8 & 39.3 \\
\hline \multirow[t]{2}{*}{5} & $S$ & 50.5 & 64.7 & 73.5 & 88.2 & 103.2 & 118.8 \\
\hline & $\mathrm{R}$ & 50.5 & 50.1 & 45.8 & 45.4 & 45.1 & 44.7 \\
\hline 6 & S & 56.7 & 66.7 & 82.4 & 91.7 & 107.3 & 115.6 \\
\hline . & $\mathrm{R}$ & 56.7 & 51.6 & 51.2 & 47.3 & 46.9 & 43.7 \\
\hline \multirow[t]{2}{*}{8} & $S$ & 62.2 & 73.8 & 91.2 & 102.0 & 119.3 & $\cdot 129.0$ \\
\hline & $\mathrm{R}$ & 62.2 & 57.1 & 56.6 & 52.5 & 52.1 & 48.7 \\
\hline \multirow[t]{2}{*}{10} & $S$ & 67.0 & 79.9 & 98.7 & 110.9 & 129.8 & 140.9 \\
\hline & $\mathrm{R}$ & 67.0 & 61.8 & 61.2 & 57.0 & 56.5 & 52.9 \\
\hline \multirow[t]{2}{*}{12} & S & 70.1 & 90.0 & 104.0 & 117.5 & 137.4 & 158.4 \\
\hline & $\mathrm{R}$ & 70.1 & 69.4 & 64.4 & 60.2 & 59.7 & 59.1 \\
\hline \multirow[t]{2}{*}{14} & $S$ & 74.8 & 96.2 & 111.1 & 125.4 & 146.7 & 169.2 \\
\hline & $\mathrm{R}$ & 74.8 & 74.1 & 68.7 & 64.1 & 63.5 & 62.8 \\
\hline \multirow[t]{2}{*}{16} & $S$ & 82.4 & 98.9 & 122.3 & 137.9 & 161.3 & 186.2 \\
\hline & $\mathrm{R}$ & 82.4 & 76.2 & 75.4 & 70.3 & 69.5 & 68.7 \\
\hline \multirow[t]{2}{*}{18} & $\mathrm{~S}$ & 83.8 & 107.8 & 133.3 & 150.3 & 175.8 & 203.0 \\
\hline & $\mathrm{R}$ & 83.8 & 82.9 & 82.0 & 76.3 & 75.4 & 74.5 \\
\hline
\end{tabular}

Note: Energy Cost $=\$ 12.00 / \mathrm{MBtu}$

Return Pipe Temperature $=250 \mathrm{~F}$ 
TABLE $\mathrm{c}-10 . \mathrm{b}$

$$
\begin{aligned}
& \text { Economic Insulation Thickness (inch) for Supply } \\
& \text { and Return Pipes for Energy Cost of } \$ 12 / \mathrm{MBtu}
\end{aligned}
$$

\begin{tabular}{ccccccc}
$\begin{array}{c}\text { Pipe } \\
\text { Size } \\
\text { (inch) }\end{array}$ & \multicolumn{6}{c}{ Process Fluid Temperature $(F)$} \\
\cline { 2 - 6 } 1 & 3.0 & 3.0 & 3.0 & 3.0 & 3.0 & 3.0 \\
2 & 3.0 & 3.0 & 3.0 & 3.5 & 3.5 & 3.5 \\
3 & 3.0 & 3.0 & 3.0 & 3.5 & 3.5 & 4.0 \\
4 & 3.5 & 3.5 & 3.5 & 3.5 & 3.5 & 4.0 \\
5 & 3.5 & 3.5 & 4.0 & 4.0 & 4.0 & 4.0 \\
6 & 3.5 & 4.0 & 4.0 & 4.5 & 4.5 & 5.0 \\
8 & 4.0 & 4.5 & 4.5 & 5.0 & 5.0 & 5.5 \\
-10 & 4.5 & 5.0 & 5.0 & 5.5 & 5.5 & 6.0 \\
12 & 5.0 & 5.0 & 5.5 & 6.0 & 6.0 & 6.0 \\
14 & 5.0 & 5.0 & 5.5 & 6.0 & 6.0 & 6.0 \\
16 & 5.0 & 5.5 & 5.5 & 6.0 & 6.0 & 6.0 \\
18 & 5.5 & 5.5 & 5.5 & 6.0 & 6.0 & 6.0
\end{tabular}

Note: Energy Cost $=\$ 12.00 / \mathrm{MBtu}$

Return Pipe Temperature $=250 \mathrm{~F}$ 
TABLE C-11.a

Maximum Allowable Heat Losses (Btu/h.ft) from Hot Water Supply

Pipe (S) and Return Pipe (R) for Energy Cost of $\$ 13 /$ MBtu

\begin{tabular}{|c|c|c|c|c|c|c|c|}
\hline \multirow{3}{*}{$\begin{array}{l}\text { Pipe } \\
\text { Size } \\
\text { (inch) }\end{array}$} & \multirow[b]{2}{*}{ Pipe } & \multicolumn{6}{|c|}{ Process Fluid Temperature (F) } \\
\hline & & & & & & & \\
\hline & Type & $\underline{250}$ & $\underline{300}$ & $\underline{350}$ & $\underline{400}$ & $\underline{450}$ & $\underline{500}$ \\
\hline \multirow[t]{2}{*}{1} & s & 24.6 & 31.4 & 38.8 & 46.5 & 54.4 & 62.5 \\
\hline & $\mathbf{R}$ & 24.6 & 24.4 & 24.3 & 24.2 & 24.0 & 23.9 \\
\hline \multirow[t]{2}{*}{2} & s & 33.1 & 42.4 & 52.4 & 57.9 & 67.7 & 77.9 \\
\hline & $\mathrm{R}$ & 33.1 & 32.9 & 32.7 & 30.0 & 29.9 & 29.7 \\
\hline \multirow[t]{2}{*}{3} & s & 41.4 & 53.1 & 65.6 & 71.9 & 84.1 & 89.7 \\
\hline & $\mathrm{R}$ & 41.4 & 41.1 & 40.8 & 37.2 & 36.9 & 34.1 \\
\hline \multirow[t]{2}{*}{4} & $S$ & 44.1 & 56.5 & 69.8 & 83.8 & 98.0 & 104.0 \\
\hline & $\mathrm{R}$ & 44.1 & 43.8 & 43.5 & 43.1 & 42.8 & 39.3 \\
\hline \multirow[t]{2}{*}{5} & $s$ & 50.5 & 59.5 & 73.5 & 88.2 & 103.2 & 118.8 \\
\hline & $\mathrm{R}$ & 50.5 & 46.1 & 45.8 & 45.4 & 45.1 & 44.7 \\
\hline \multirow[t]{2}{*}{6} & s & 56.7 & 66.7 & 76.4 & 91.7 & 107.3 & 115.6 \\
\hline & $\mathrm{R}$ & 56.7 & 51.6 & 47.6 & 47.3 & 46.9 & 43.7 \\
\hline \multirow[t]{2}{*}{8} & $S$ & 62.2 & 73.8 & 85.1 & 102.0 & 112.1 & 129.0 \\
\hline & $\mathrm{R}$ & 62.2 & 57.1 & 53.0 & 52.5 & 49.1 & 48.7 \\
\hline \multirow[t]{2}{*}{10} & $S$ & 67.0 & 79.9 & 92.5 & 110.9 & 122.3 & 140.9 \\
\hline & $\mathrm{R}$ & 67.0 & 61.8 & 57.4 & 57.0 & 53.4 & 52.9 \\
\hline \multirow[t]{2}{*}{12} & $S$ & 70.1 & 84.1 & 104.0 & 117.5 & 137.4 & 158.4 \\
\hline & $R$ & 70.1 & 65.0 & 64.4 & 60.2 & 59.7 & 59.1 \\
\hline \multirow[t]{2}{*}{14} & S & 74.8 & 89.8 & 104.5 & 125.4 & 146.7 & 169.2 \\
\hline & $\mathrm{R}$ & 74.8 & 69.3 & 64.7 & 64.1 & 63.5 & 62.8 \\
\hline \multirow[t]{2}{*}{16} & $S$ & 76.9 & 98.9 & 114.9 & 137.9 & 161.3 & 186.2 \\
\hline & $\mathrm{R}$ & 76.9 & 76.2 & 71.0 & 70.3 & 69.5 & 68.7 \\
\hline \multirow[t]{2}{*}{18} & $S$ & 83.8 & 107.8 & 125.1 & 150.3 & 175.8 & $20 \cdot 3.0$ \\
\hline & $\mathrm{R}$. & 83.8 & 82.9 & 77.1 & 76.3 & 75.4 & 74.5 \\
\hline
\end{tabular}

Note: Energy Cost $=\$ 13.00 / \mathrm{MBtu}$

Return Pipe Temperature $=250 \mathrm{~F}$ 
TABLE $\mathrm{C}-11 . \mathrm{b}$

Economic Insulation Thickness (inch) for Supply and Return Pipes for Energy Cost of $\$ 13 / \mathrm{MBtu}$

\begin{tabular}{ccccccc}
$\begin{array}{c}\text { Pipe } \\
\begin{array}{c}\text { Size } \\
\text { (inch) }\end{array}\end{array}$ & $\underline{5}$ Process & Fluid & Temperature (F) & \\
\cline { 2 - 6 } 1 & 3.0 & 3.0 & 3.0 & 3.0 & 3.0 & 3.0 \\
2 & 3.0 & 3.0 & 3.0 & 3.5 & 3.5 & 3.5 \\
3 & 3.0 & 3.0 & 3.0 & 3.5 & 3.5 & 4.0 \\
4 & 3.5 & 3.5 & 3.5 & 3.5 & 3.5 & 4.0 \\
5 & 3.5 & 4.0 & 4.0 & 4.0 & 4.0 & 4.0 \\
6 & 3.5 & 4.0 & 4.5 & 4.5 & 4.5 & 5.0 \\
8 & 4.0 & 4.5 & 5.0 & 5.0 & 5.5 & 5.5 \\
-10 & 4.5 & 5.0 & 5.5 & 5.5 & 6.0 & 6.0 \\
12 & 5.0 & 5.5 & 5.5 & 6.0 & 6.0 & 6.0 \\
14 & 5.0 & 5.5 & 6.0 & 6.0 & 6.0 & 6.0 \\
16 & 5.5 & 5.5 & 6.0 & 6.0 & 6.0 & 6.0 \\
18 & 5.5 & 5.5 & 6.0 & 6.0 & 6.0 & 6.0
\end{tabular}

Note: Energy Cost $=\$ 13.00 / \mathrm{MBtu}$

Return Pipe Temperature $=250 \mathrm{~F}$ 
TABLE $C-12$. a

Maximum Allowable Heat Losses (Btu/h.ft) from Hot Water Supply Pipe (S) and Return Pipe (R) for Energy Cost of $\$ 14 / \mathrm{MBtu}$

\begin{tabular}{|c|c|c|c|c|c|c|c|}
\hline \multirow{3}{*}{$\begin{array}{l}\text { Pipe } \\
\text { Size } \\
\text { (inch) } \\
\end{array}$} & \multirow[b]{2}{*}{ Pipe } & \multicolumn{6}{|c|}{ Process Fluid Temperature (F) } \\
\hline & & & & & & & \\
\hline & Type & $\underline{250}$ & $\underline{300}$ & $\underline{350}$ & 400 & $\underline{450}$ & $\underline{500}$ \\
\hline \multirow[t]{2}{*}{1} & $S$ & 24.6 & 31.4 & 38.8 & 46.5 & 54.4 & 62.5 \\
\hline & $\mathbf{R}$ & 24.6 & 24.4 & 24.3 & 24.2 & 24.0 & 23.9 \\
\hline \multirow[t]{2}{*}{2} & $\mathrm{~s}$ & 33.1 & 42.4 & 48.3 & 57.9 & 67.7 & 77.9 \\
\hline & $\mathrm{R}$ & 33.1 & 32.9 & 30.2 & 30.0 & 29.9 & 29.7 \\
\hline \multirow[t]{2}{*}{3} & $S$ & 41.4 & 53.1 & 60.0 & 66.7 & 78.0 & 89.7 \\
\hline & $\mathrm{R}$ & 41.4 & 41.1 & 37.4 & 34.6 & 34.3 & 34.1 \\
\hline \multirow[t]{2}{*}{4} & $S$ & 44.1 & 56.5 & 69.8 & 77.3 & 90.4 & 104.0 \\
\hline & $\mathrm{R}$ & 44.1 & 43.8 & 43.5 & 39.9 & 39.6 & 39.3 \\
\hline \multirow[t]{2}{*}{5} & $S$ & 50.5 & 59.5 & 73.5 & 88.2 & 103.2 & 118.8 \\
\hline & $R$ & 50.5 & 46.1 & 45.8 & 45.4 & 45.1 & 44.7 \\
\hline \multirow[t]{2}{*}{6} & $S$ & 52.0 & 61.9 & 76.4 & 91.7 & 107.3 & 115.6 \\
\hline & $\mathrm{R}$ & 52.0 & 48.0 & 47.6 & 47.3 & 46.9 & 43.7 \\
\hline \multirow[t]{2}{*}{8} & $S$ & 57.5 & 68.8 & 85.1 & 95.8 & 112.1 & 121.9 \\
\hline & $\mathbf{R}$ & 57.5 & 53.4 & 53.0 & 49.5 & 49.1 & 46.1 \\
\hline \multirow[t]{2}{*}{10} & $S$ & 62.3 & 74.8 & 92.5 & 104.6 & 122.3 & 140.9 \\
\hline & $\mathrm{R}$ & 62.3 & 57.9 & 57.4 & 53.8 & 53.4 & 52.9 \\
\hline \multirow[t]{2}{*}{12} & $S$ & 65.5 & 84.1 & 97.9 & 117.5 & 137.4 & 158.4 \\
\hline & R & 65.5 & 65.0 & 60.7 & 60.2 & 59.7 & 59.1 \\
\hline \multirow[t]{2}{*}{14} & $S$ & 70.0 & 89.8 & 104.5 & 125.4 & 146.7 & 169.2 \\
\hline & $\mathbf{R}$ & 70.0 & 69.3 & 64.7 & 64.1 & 63.5 & 62.8 \\
\hline \multirow[t]{2}{*}{16} & $S$ & 76.9 & 98.9 & 114.9 & 137.9 & 161.3 & 186.2 \\
\hline & $\mathbf{R}$ & 76.9 & 76.2 & 71.0 & 70.3 & 69.5 & 68.7 \\
\hline \multirow[t]{2}{*}{18} & $S$ & 83.8 & 107.8 & 125.1 & 150.3 & 175.8 & 203.0 \\
\hline & $\mathrm{R}$ & 83.8 & 82.9 & 77.1 & 76.3 & 75.4 & 74.5 \\
\hline
\end{tabular}

Note: Energy Cost $=\$ 14.00 / \mathrm{MBtu}$

Return Pipe Temperature $=250 \mathrm{~F}$ 
TABLE $c-12 \cdot b$

Economic Insulation Thickness (inch) for Supply and Return Pipes for Energy Cost of $\$ 14 / \mathrm{MBtu}$

\begin{tabular}{ccccccc}
$\begin{array}{c}\text { Pipe } \\
\text { Size } \\
\text { (inch) }\end{array}$ & $\underline{5}$ Process & Fluid & Temperature $(F)$ & \\
\cline { 2 - 6 } 1 & 3.0 & 3.0 & 3.0 & 3.0 & 3.0 & 3.0 \\
2 & 3.0 & 3.0 & 3.5 & 3.5 & 3.5 & 3.5 \\
3 & 3.0 & 3.0 & 3.5 & 4.0 & 4.0 & 4.0 \\
4 & 3.5 & 3.5 & 3.5 & 4.0 & 4.0 & 4.0 \\
5 & 3.5 & 4.0 & 4.0 & 4.0 & 4.0 & 4.0 \\
6 & 4.0 & 4.5 & 4.5 & 4.5 & 4.5 & 5.0 \\
8 & 4.5 & 5.0 & 5.0 & 5.5 & 5.5 & 6.0 \\
-10 & 5.0 & 5.5 & 5.5 & 6.0 & 6.0 & 6.0 \\
12 & 5.5 & 5.5 & 6.0 & 6.0 & 6.0 & 6.0 \\
14 & 5.5 & 5.5 & $6: 0$ & 6.0 & 6.0 & 6.0 \\
16 & 5.5 & 5.5 & 6.0 & 6.0 & 6.0 & 6.0 \\
18 & 5.5 & 5.5 & 6.0 & 6.0 & 6.0 & 6.0
\end{tabular}

Note: Energy Cost $=\$ 14.00 / \mathrm{MBtu}$

Return Pipe Temperature $=250 \mathrm{~F}$ 
TABLE $\quad C-13 \cdot a$

Maximum Allowable Heat Losses (Btu/h.ft) from Hot Water Supply Pipe (S) and Return Pipe (R) for Energy Cost of $\$ 15 / \mathrm{MBtu}$

\begin{tabular}{|c|c|c|c|c|c|c|c|}
\hline \multirow{3}{*}{$\begin{array}{c}\text { Pipe } \\
\text { Size } \\
\text { (inch) } \\
\end{array}$} & \multirow[b]{2}{*}{ Pipe } & \multicolumn{6}{|c|}{ Process Fluid Temperature (F) } \\
\hline & & & & & & & \\
\hline & Type & $\underline{250}$ & $\underline{300}$ & $\underline{350}$ & 400 & 450 & $\underline{500}$ \\
\hline \multirow[t]{2}{*}{1} & S & 24.6 & 31.4 & 38.8 & 46.5 & 54.4 & 62.5 \\
\hline & $\mathrm{R}$ & 24.6 & 24.4 & 24.3 & 24.2 & 24.0 & 23.9 \\
\hline \multirow[t]{2}{*}{2} & $S$ & 33.1 & 42.4 & 48.3 & 57.9 & 67.7 & 77.9 \\
\hline & $\mathrm{R}$ & 33.1 & 32.9 & 30.2 & 30.0 & 29.9 & 29.7 \\
\hline \multirow[t]{2}{*}{3} & $S$ & 41.4 & 53.1 & 60.0 & 66.7 & 78.0 & 89.7 \\
\hline & $\mathbf{R}$ & 41.4 & 41.1 & 37.4 & 34.6 & 34.3 & 34.1 \\
\hline \multirow[t]{2}{*}{4} & $S$ & 44.1 & 56.5 & 69.8 & 77.3 & 90.4 & 104.0 \\
\hline & $\mathrm{R}$ & 44.1 & 43.8 & 43.5 & 39.9 & 39.6 & 39.3 \\
\hline \multirow[t]{2}{*}{5} & $S$ & 50.5 & 59.5 & 73.5 & 88.2 & 103.2 & 118.8 \\
\hline & $\mathrm{R}$ & 50.5 & 46.1 & 45.8 & 45.4 & 45.1 & 44.7 \\
\hline \multirow[t]{2}{*}{6} & $S$ & 52.0 & 61.9 & 76.4 & 91.7 & 107.3 & 115.6 \\
\hline & R & 52.0 & 48.0 & 47.6 & 47.3 & 46.9 & 43.7 \\
\hline \multirow[t]{2}{*}{8} & $S$ & 57.5 & 68.8 & 85.1 & 95.8 & 112.1 & 121.9 \\
\hline & $\mathrm{R}$ & 57.5 & 53.4 & 53.0 & 49.5 & 49.1 & 46.1 \\
\hline \multirow[t]{2}{*}{10} & $S$ & 62.3 & 74.8 & 92.5 & 104.6 & 122.3 & 140.9 \\
\hline & R & 62.3 & 57.9 & 57.4 & 53.8 & 53.4 & 52.9 \\
\hline \multirow[t]{2}{*}{12} & $S$ & 65.5 & 84.1 & 97.9 & 117.5 & 137.4 & 158.4 \\
\hline & $\mathrm{R}$ & 65.5 & 65.0 & 60.7 & 60.2 & 59.7 & 59.1 \\
\hline \multirow[t]{2}{*}{14} & $S$ & 70.0 & 89.8 & 104.5 & 125.4 & 146.7 & 169.2 \\
\hline & $\mathrm{R}$ & 70.0 & 69.3 & 64.7 & 64.1 & 63.5 & 62.8 \\
\hline \multirow[t]{2}{*}{16} & $S$ & 76.9 & 92.9 & 114.9 & 137.9 & 161.3 & 186.2 \\
\hline & $\mathrm{R}$ & 76.9 & 71.7 & 71.0 & 70.3 & 69.5 & 68.7 \\
\hline \multirow[t]{2}{*}{18} & S & 83.8 & 101.2 & 125.1 & 150.3 & 175.8 & 203.0 \\
\hline & $\mathbf{R}$ & 83.8 & 78.0 & 77.1 & 76.3 & 75.4 & 74.5 \\
\hline
\end{tabular}


TABLE C-13.b

Economic Insulation Thickness (inch) for Supply and Return Pipes for Energy Cost of $\$ 15 / \mathrm{MBtu}$

\begin{tabular}{|c|c|c|c|c|c|c|}
\hline \multirow{2}{*}{$\begin{array}{l}\text { Pipe } \\
\text { Size } \\
\text { (inch) } \\
\end{array}$} & \multicolumn{6}{|c|}{ Process Fluid Iemperature $(E)$} \\
\hline & $\underline{250}$ & $\underline{300}$ & $\underline{350}$ & $\underline{400}$ & $\underline{450}$ & $\underline{500}$ \\
\hline 1 & 3.0 & 3.0 & 3.0 & 3.0 & 3.0 & 3.0 \\
\hline 2 & 3.0 & 3.0 & 3.5 & 3.5 & 3.5 & 3.5 \\
\hline 3 & 3.0 & 3.0 & 3.5 & 4.0 & 4.0 & 4.0 \\
\hline 4 & 3.5 & 3.5 & 3.5 & 4.0 & 4.0 & 4.0 \\
\hline 5 & 3.5 & 4.0 & 4.0 & 4.0 & 4.0 & 4.0 \\
\hline 6 & 4.0 & 4.5 & 4.5 & 4.5 & 4.5 & 5.0 \\
\hline 8 & 4.5 & 5.0 & 5.0 & 5.5 & 5.5 & 6.0 \\
\hline .10 & 5.0 & 5.5 & 5.5 & 6.0 & 6.0 & 6.0 \\
\hline 12 & 5.5 & 5.5 & 6.0 & 6.0 & 6.0 & 6.0 \\
\hline 14 & 5.5 & 5.5 & 6.0 & 6.0 & 6.0 & 6.0 \\
\hline 16 & 5.5 & 6.0 & 6.0 & 6.0 & 6.0 & 6.0 \\
\hline 18 & 5.5 & 6.0 & 6.0 & 6.0 & 6.0 & 6.0 \\
\hline
\end{tabular}

Note: Energy Cost $=\$ 15.00 / \mathrm{MBtu}$

Return Pipe Temperature $=250 \mathrm{~F}$ 
NBS-114A (REV. 2.8C)

U.S. OEPT. OF COMM.

BIBLIOGRAPHIC DATA

SHEET (See instructions)

4. TITLE AND SUBTITLE

Calculations of Maximum Allowable Heat Losses for Various

Stallow Trenct. Heat Distributior. Systens

5. $\operatorname{AUTHOR}(S)$

Jin B. Fang

6. PERFORMING ORGANIZATION (If joint or other thon NBS, see instructions)

7. Coneracd Grane No.

MATIONAL BUREAU OF STANDARDS

DEPARTMENT OF COMMERCE

WASHINGTON, D.C. 20234

9. SPONSORING ORGANIZATION NAME AND COMPLETE ADDRESS (Street. City, Stote, ZIP)

Tri-Service Building Materials Committee

Headquarters, U.S. Army Corps of Engineers

Washington, DC 20314-1000

10. SUPPLEMENTARY NOTES

5 Document describes a computer program; SF-185, FIPS Software Summary, is attached.

11. ABSTRACT (A 200-word or less factual summary of most significant information. If document includes a significant bibliography or literoture survey. mention it here)

The calculation of heat losses for shallow trench underground heat distribution systems was performed using a finite element computer program. The finite element analysis solved a two-dimensional steady-state heat transfer problem for two insulated pipes in a rectangular trench with surrounding soil. A life-cycle cost analysis was performed to determine the cost of construction and annual energy cost associated with pipe heat loss for underground concrete trench systems of different trench dimensions and insulated pipe sizes. Procedures for determining the pipe heat losses associated with the minimum life-cycle cost and the corresponding optimum insulation thickness for shallow trench distribution systems are presented. Based on the results of the economic analysis, the maximum allowable heat losses and the insulation thickness for underground pipes were determined and tabulated for a range of pipe sizes and fluid temperatures, various levels of fuel costs, and for a known undisturbed earth temperature and soil thermal conductivity.

12. KEY WORDS (Six to twelve entries; alphobetical order; copitalize only proper names; and seporate key words by semicolons) district heating and cooling; finite element method; fuel energy cost; heat loss; Iife-cycle cost analysis; pipe insulation thickness; shallow trench; underground heat distribution system.

13. AVAILABILITY

xxy Unlimited

For Official Distribution. Do Not Release to NTIS

- Order From Suberintendent of Documents, U.S. Government Printing Office, Washington, D.C.

20402.

XX Order From National Technical Information Service (NTIS), Springfield, VA. 22161

14. NO. OF

PRINTED PAGES

118

15. Price

$\$ 18.95$ 

